\title{
A Mobile Healthcare Solution for Ambient Assisted Living Environments
}

\section{Daniel Fernando Mendes Rodrigues}

Submitted to the University of Beira Interior in applying for the

Degree of Master of Science in Informatics Engineering

Supervised by Prof. Dr. Joel José Puga Coelho Rodrigues

\author{
Departamento de Informática \\ University of Beira Interior \\ Covilhã, Portugal \\ http://www.di.ubi.pt
}



Part of this work has been supported by Instituto de Telecomunicações, Next Generation Networks and Applications Group (NetGNA), Portugal, by National Funding from the FCT - Fundação para a Ciência e Tecnologia through the PEst-OE/EEI/LA0008/2013 Project, and by the AAL4ALL (Ambient Assisted Living for All), project cofounded by COMPETE under FEDER via QREN Programme.
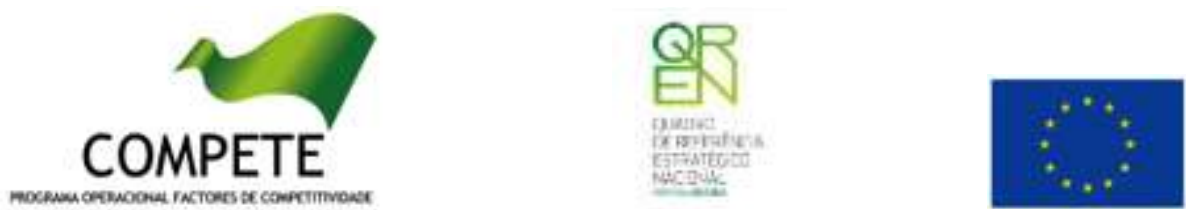

UNIĀO EUROPELAA

Funtso Eimspeu

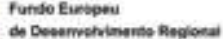





\section{Acknowledgements}

First of all, I wish to thank Professor Joel José Puga Coelho Rodrigues for giving me the chance and the challenge to join the Next Generation Networks and Applications Research Group (NetGNA), for his advice and continuous support and inspiration throughout the process of supervising my Master thesis.

I am most grateful to the University of Beira Interior, Instituto de Telecomunicações, Next Generation Networks and Applications Group (NetGNA), Covilhã Delegation, Portugal, in the framework by the AAL4ALL (Ambient Assisted Living for All) project, and by all the multiple kinds of support that allowed me to perform this Master's thesis.

Many thanks to all the members of NetGNA, particularly to Edgar Horta, Fábio Guedes, Bruno Silva, Ângelo Fonseca, Sandra Sendra, Susana Costa and João Isento.

Finally, my eternal gratitude to my parents Fernando Rodrigues, Ascenção Neto, my sister Cristina Rodrigues and also to my girlfriend Carla Neves as well as to my closest family for all the constant encouragement, patience and support and to all my friends not mentioned here. 



\section{Abstract}

Elderly people need regular healthcare services and, several times, are dependent of physicians' personal attendance. This dependence raises several issues to elders, such as, the need to travel and mobility support. Ambient Assisted Living (AAL) and Mobile Health (m-Health) services and applications offer good healthcare solutions that can be used both on indoor and in mobility environments. This dissertation presents an ambient assisted living (AAL) solution for mobile environments.

It includes elderly biofeedback monitoring using body sensors for data collection offering support for remote monitoring. The used sensors are attached to the human body (such as the electrocardiogram, blood pressure, and temperature). They collect data providing comfort, mobility, and guaranteeing efficiency and data confidentiality. Periodic collection of patients' data is important to gather more accurate measurements and to avoid common risky situations, like a physical fall may be considered something natural in life span and it is more dangerous for senior people. One fall can out a life in extreme cases or cause fractures, injuries, but when it is early detected through an accelerometer, for example, it can avoid a tragic outcome.

The presented proposal monitors elderly people, storing collected data in a personal computer, tablet, or smartphone through Bluetooth. This application allows an analysis of possible health condition warnings based on the input of supporting charts, and real-time bio-signals monitoring and is able to warn users and the caretakers. 
These mobile devices are also used to collect data, which allow data storage and its possible consultation in the future. The proposed system is evaluated, demonstrated and validated through a prototype and it is ready for use. The watch Texas ez430-Chronos, which is capable to store information for later analysis and the sensors Shimmer who allow the creation of a personalized application that it is capable of measuring biosignals of the patient in real time is described throughout this dissertation. 


\section{Keywords}

Mobile wearable sensors, Ambient Assisted Living, Technology Acceptance, Safety Analysis, Mobile Computing, Biofeedback Monitoring, m-Health, Body Sensor Networks, Healthcare Application. 



\section{Contents}

Acknowledgements ........................................... i

Abstract............................................................. iii

Keywords .........................................................v

Contents ........................................................... vii

List of Figures .................................................. xi

List of Tables ..................................................... xiii

List of Equations ............................................... xv

Acronyms .........................................................xvii

1. Introduction ....................................... 1

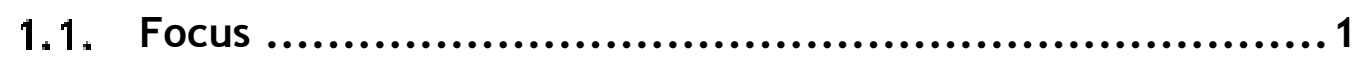

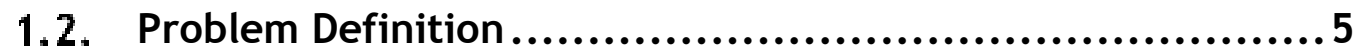

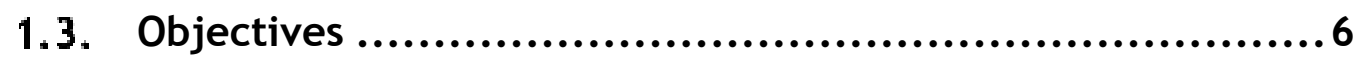

1.4. Main Contributions ....................................................

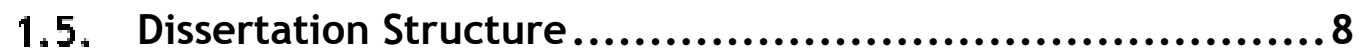

2. Related Work ....................................... 11

2.1. Anywhere, Anytime for Anyone.................................. 12

2.2. Security, Privacy and Confidentiality of Information in Ambient Assisted Living .................................................. 13

2.2.1. Direct Advantages and Disadvantages of AAL............ 15

2.2.2. Privacy and Confidentiality ............................... 16 
2.2.3. Security, Safety and Comfort ......................... 17

2.2.4. Reviews............................................. 18

2.3. Analysis in Healthcare Technologies ........................ 19

2.3.1. Assistive Technologies and Electronic Services in AAL environments................................................ 20

2.3.2. Smart Artefacts and Mobile Devices ..................... 21

2.3.3. Wearables Sensors and/or Embedded Mobile Device Sensors

2.3.4. Smart Environments and Smart Homes (SHs) .............. 23

2.3.5. Reviews.......................................... 24

2.3. Health Sensor Systems .................................. 26

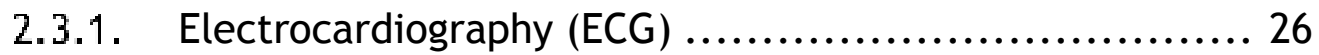

2.3.2. Electromyography (EMG) ............................ 27

2.3.3. Galvanic Skin Response (GSR) or Electro Dermal Activity

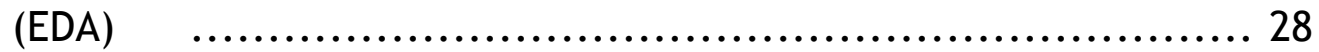

2.3.4. Accelerometer ......................................... 29

2.4. Falls Detection and Prevention Approaches .................. 30

2.4.1. X Health Systems .................................... 31

2.5. Summary ................................................... 32

3. Requirement Analysis .............................. 33

3.1. Essential Modeling Requirements ........................... 34

3.2. Behavioural Diagrams ...................................... 34

3.2.1. Use Case Diagrams.................................... 35

3.2.2. Activity Diagrams ................................... 37

3.3. Structural Diagrams ....................................... 43

3.4. Used Technologies ........................................ 45

3.5. Summary ................................................ 48

4. Devices used on the Prototype ...................... 49

4.1. Shimmer Research Platinum ............................. 50

4.1.1. Arrangement of the Sensors in Patients Body ............. 51

4.2. Texas eZ430-Chronos ........................................ 54 
4.2.1. Arrangement of the Sensors in Patients Body ............. 56

4.3. Summary ................................................. 57

5. AAL Mobile Solution Demonstration ................... 59

5.1. System Architecture ........................................... 59

5.1.1. System Architecture - Watch .......................... 61

5.2. Graphical Interface .........................................6 61

5.2.1. Graphical Interface - Watch............................ 64

5.3. Biofeedback Monitoring Tool................................ 65

5.3.1. Biofeedback Monitoring Tool - Watch ................... 66

5.4. Falls Detection with External Alerts.......................... 68

5.5. Summary ............................................... 69

6. System Validation and Performance Evaluation....... 71

6.1. Collecting Bio-signals and their Analysis..................... 72

6.2. Falls Detection and their Analysis ......................... 76

6.3. Summary .................................................... 78

7. Conclusions and Future Work .......................... 79

7.1. Conclusions ............................................... 79

7.2. Future Work............................................ 81

References $\ldots \ldots \ldots \ldots \ldots \ldots \ldots \ldots \ldots \ldots \ldots \ldots \ldots \ldots \ldots \ldots \ldots \ldots \ldots \ldots \ldots . . \ldots 3$

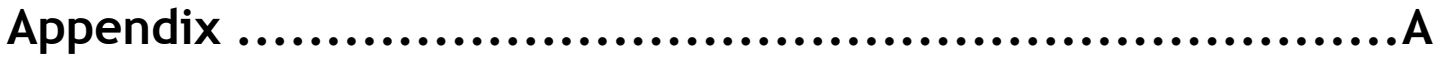





\section{List of Figures}

FIGURE 1 ILLUSTRATES TYPICAL MOBILE HEALTH SYSTEMS, CONTAINING ONE OR MORE MONITORING AND/OR SURVEILLANCE RESOURCES AND DEVICES, SUCH AS WEARABLE SENSORS. THEY SHOULD BE CONNECTED TO THE INTERNET TO ALLOW INFORMATION SENDING, DATA STORAGE AND RETRIEVAL.

FIGURE 2 - ILLUSTRATION OF RANGE (CONTINUITY AND/OR INTENSITY) OF PERSONA PLATFORM........... 14

FIGURE 3 - ILLUSTRATION OF ECG SIGNAL DETECTION. .......................................... 27

FIGURE 4 - ILLUSTRATION OF ELECTROMYOGRAPHY TEST. ........................................ 28

FIGURE 5 - ILLUSTRATION OF AN EDA OR GSR SENSOR. ......................................... 29

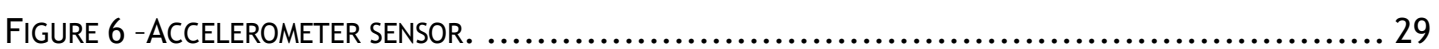

FIGURE 7 - APPLICATION USE CASE DIAGRAM FOR TEXAS INSTRUMENTS EZ430-CHRONOS.................. 36

FIGURE 8 - APPLICATION USE CASE DIAGRAM fOR SHIMMER SENSORS. ................................ 37

FigURE 9 - ACTIVITY DIAGRAM ILLUSTRATING THE ECG AND TEMPERATURE PREVENTION, AND DETECTION

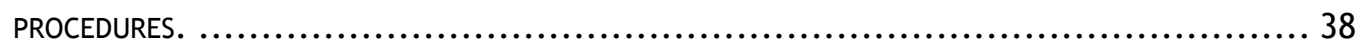

FIGURE 10 - Falls DeteCtION ACTIVITY DIAGRAM......................................... 40

Figure 11 - Bluetooth Connection, Receive, Store and processing data, other Show Charts

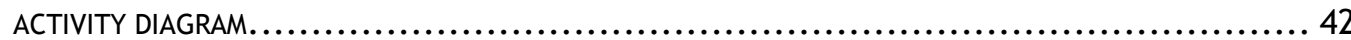

Figure 12 - StRuctural Diagram With BLUETOOTH CONNECTION FOR HEALTH SENSORS, FALLS DETECTION, GPS LOCATION AND TO DISPLAY HEALTH DATA CHARTS. ................................ 44

FiguRE 13 - DeVELoPMENT Of THE APPLICATION ON IDE NetBeans. ............................. 46

FIGURE 14 - ILLUSTRATION OF THE ANDROID OS ARCHITECTURE................................ 46

FIGURE 15 - DEVELOPMENT ENVIRONMENT ADT. ............................................ 48

Figure 16 - Shimmer Research Platinum Kit. ........................................... 50

Figure 17 - IlLUSTRATION OF THE BODY SENSORS - SHIMMER: A) EleCtro-CARDIOGRAM; B) TEMPERATURE;

C) EleCTRODES FOR EleCTRO-CARDIOGRAM. ....................................... 52

FIGURE 18 - MATERIAL USED FOR THE DEVELOPMENT OF THE SOLUTION............................ 53

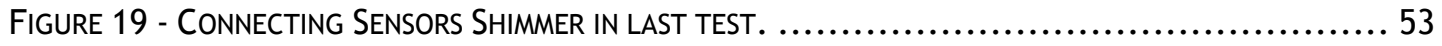

Figure 20 - Texas eZ430-Chronos Development Kit....................................... 54

Figure 21 - Placement of the Sensors in the Human Body. ................................ 56

FIGURE 22 - ILLUSTRATION OF THE SYSTEM ARCHITECTURE, USING ECG AND TEMPERATURE BODY SENSORS AND EXTERNAL ACCESS THROUGH WEB SERVICES FOR DATA VISUALIZATION AND STORAGE...............6 60 
FIGURE 23 - ILLUSTRATION OF THE OFFLINE SYSTEM ARCHITECTURE CONSIDERING THE CAPTURE OF BLOOD VOLUME PULSE (BVP) AND BODY TEMPERATURE VALUES. ............................... 61

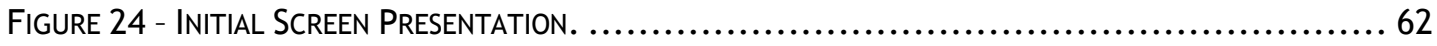

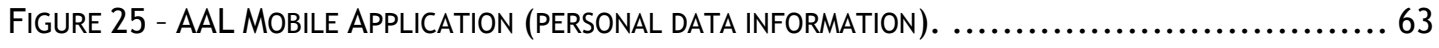

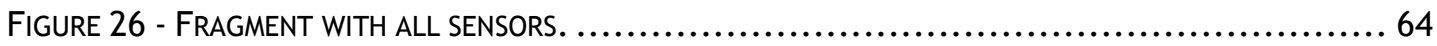

FigURE 27 - INITIAL SCREEN PRESENTATION (PATIENT INFORMATION)............................... 65

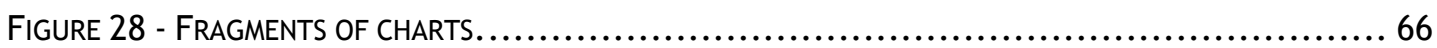

FIGURE 29 - EXAMPLE OF GRAPH APPLICATION AND RESPECTIVE INFORMATION. ........................ 67

FIGURE 30 - EXAMPLE OF A DATA FILTER THROUGH THE INTENSITY LEVEL. ........................... 68

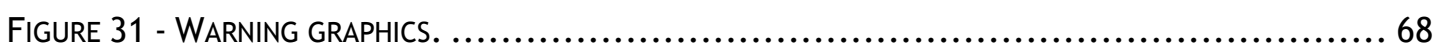

Figure 32- USer Falls Detection Notification and Red Signal alert. ............................ 69

FigURE 33 - PROPOSED APPLICATION FOR THE TEXAS INSTRUMENTS WATCH SHOWING COLLECTED DATA FROM TEMPERATURE AND HEART RATE FROM A GIVEN PATIENT. .................................... 73

FIGURE 34 - COMPARISON OF AVERAGE ALERTS VALUES TO EACH LEVEL OF OBSERVATION SYSTEM. (WATCH AND

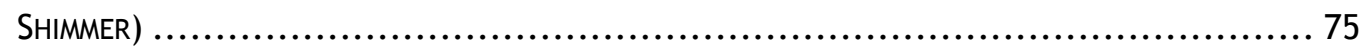

FiguRe 35 - Bar Chart Values Ordered. ................................................ 77

FIGURE 36 - COMPARISON OF AVERAGE ALERTS VALUES TO EACH SIMULATION. ........................... 77 


\section{List of Tables}

TABLE 1. ACTORS, BEHAVIORS, AND REQUIREMENTS THE SYSTEM MUST IMPLEMENT ...................... 35

Table 2. Total Number of Alerts Per LeVel of Surveillance (LOW, normal and CRItical) With a STANDARD DEVIATION OF 10\% AND 15\% CONSIDERING THE TI WATCH AND SHIMMER SENSORS, FOR THE 30

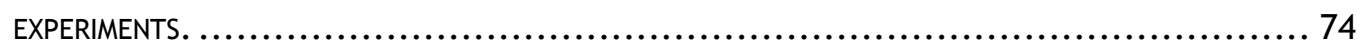

TABLE 3. EXPERIMENTAL ReSUltS CONTROL TABLE............................................... 76 



\section{List of Equations}

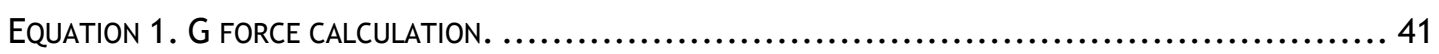





\section{Acronyms}

\begin{tabular}{|c|c|c|}
\hline A & $:$ & Ampere \\
\hline $\mathrm{AAL}$ & : & Ambient Assisted Living \\
\hline ACC & $:$ & Accelerometers \\
\hline AdAPT & $:$ & A Dynamic Approach for Activity Prediction and Tracking \\
\hline ADC & $:$ & Analog-to-Digital Converter \\
\hline ADL & $:$ & Activities of Daily Living \\
\hline ADT & $:$ & Android Developer Tools \\
\hline Aml & $:$ & Ambient Intelligent \\
\hline AmIAL & $:$ & Ambient Intelligent Assisted Living \\
\hline API & $:$ & Application Programming Interface \\
\hline ARAS & $:$ & Activity Recognition with Ambient Sensing \\
\hline ARM & $:$ & Ambient Health Monitoring \\
\hline BS & $:$ & Body Sensor \\
\hline BSN & $:$ & Body Sensor Network \\
\hline BVP & $:$ & Blood Volume Pressure \\
\hline Bit & $:$ & Binary Digit \\
\hline CPU & $:$ & Central Processing Unit \\
\hline $\mathrm{CHF}$ & $:$ & Chronic Heart Failure \\
\hline DAC & $:$ & Digital-to-Analog Converter \\
\hline DoF & $:$ & Degrees of Freedom \\
\hline ECG & $:$ & Electrocardiography \\
\hline EDA & $:$ & Electro Dermal Activity \\
\hline EMG & $:$ & Electromyography \\
\hline e-mail & : & Electronic Mail \\
\hline
\end{tabular}




\begin{tabular}{|c|c|c|}
\hline GPS & $:$ & Global Position System \\
\hline GSR & $:$ & Galvanic Skin Response \\
\hline GUI & $:$ & Graphical User Interface \\
\hline GYR & $:$ & Gyroscope \\
\hline HRM & : & Heart Rate Monitor \\
\hline $\mathrm{Hz}$ & : & Hertz (International Unit of Frequency) \\
\hline IDE & $:$ & Integration Development Environment \\
\hline ICT & : & Information and Communication(s) Technology(ies) \\
\hline IOT & $:$ & Internet of Things \\
\hline IT & $:$ & Information Technology \\
\hline JDK7 & : & Java SE Development Kit 7 \\
\hline LAN & : & Local Area Network \\
\hline MAS & : & Multi-Agent System \\
\hline NetGNA & : & Next Generation Networks and Applications Group \\
\hline PC & : & Personal Computer \\
\hline PERS & : & Personal Emergency Response System \\
\hline PERSONA & $:$ & Perceptive Spaces prOmoting iNdependent Aging \\
\hline PHM & $:$ & Pervasive Healthcare Monitoring \\
\hline PLUX & $:$ & Enterprise PLUX Wireless Biosignals S.A. \\
\hline QoL & $:$ & Quality of Life \\
\hline RE & $:$ & Requirements Engineering \\
\hline REST & $:$ & Representational State Transfer \\
\hline $\mathrm{RF}$ & $:$ & Radio Frequency \\
\hline SD Card & $:$ & Secure Digital Card \\
\hline SDK & : & Software Development Kit \\
\hline SHIMMER & : & $\begin{array}{l}\text { Sensing Health with Intelligence Modularity, Mobility and } \\
\text { Experimental Reusability }\end{array}$ \\
\hline SMS & $:$ & Short Message Service \\
\hline UI & $:$ & User Interface \\
\hline UML & $:$ & Unified Modelling Language \\
\hline USB & $:$ & Universal Serial Bus \\
\hline $\mathrm{V}$ & : & Volt (International Unit of Electric Potential) \\
\hline
\end{tabular}


VS

VWs

WAN

WLAN

WS

WSN

$\mathrm{XML}$
: Vital Sign Sensors

: Virtual Worlds

: Wireless Area Network

: Wireless Local Area Network

: Web Service

: Wireless Sensor Networks

: $\quad$ eXtended Markup Language 



\section{Introduction}

This first chapter describes the work developed to contextualize concepts and all the difficult processes, including objectives and motivations that are the main focus in Section 1.1 of this dissertation. The problem definition presented in Section 1.2 is intended to clarify the objectives of AAL mobile solutions. To accomplish the study goals mentioned in Section 1.3 (and knowing that the concepts have already been applied) I can only implement a few tasks in a limited time. Section 1.4 enumerates the main contributions for the state of the art. These contributions are included in scientific paper. This dissertation is organized in seven chapters briefly summarized in Section 1.5.

\subsection{Focus}

In the last decade several studies are showing that elderly population is quickly increasing. It is expected that about 2 billion people will be age 60 or older by 2050 [1]. In 2010, it was observed that $17.4 \%$ of the 27 European countries' population was over 65 years old and experts foresaw that, in 2060, this value will attain $30.0 \%$ [2]. Nowadays, humans tend on the need and social duty to help their parents, but overall a big part of our society tends to forget them. For instance, according to the World Health Organization, about $15 \%$ of the world's population (about 1 billion people) have some type of disability [3]. Nowadays the family core is much smaller. In fact, the model of the large family is vanishing and 
children are leaving their parents and starting to live on their own much later due to the world's economic situation. Elder people that need regular healthcare services also lives alone and nearly 10 million elderly Americans over 65 years old live by their own [4].

This emergent reality presents several challenges and opportunities for new and innovative healthcare services. The Information Technology (IT) innovation has tried to take advantage of this fact and is directing some resources and competencies to the elderly. These solutions aim to provide an assisted life style, without forgetting their health, well-being, autonomy, safety and preventing their isolation by connecting them with relatives and the community.

Currently, there is a significant growing market of small sensors embedded in clothing, jewelry, watches, shoes, phones, and other mobile devices like the Chair-type Interface [5], and other objects that elderly use on a daily basis. An elderly diagnosed with dementia would wear these small sensors allowing the assessment of health parameters without the need to go to a medical doctor office or to a clinical support. Therefore, these innovative smart care technologies allow improvements in access to care, overhaul quality and these approaches try to obtain a significant improvement in terms of quality of life (QoL), reducing interventions to support the elderly in their lifetime. This independence, efficiency and productivity of the health sector allows reducing costs for the health insurance system.

Embedded health sensors are very important because they may be able to assist people using the sensors' data by initiating an SOS phone call or even producing and sending an automatic text message. Also this information can be used as a simple query to access the historical data in a mobile application. These wearable computing technologies enable mobility and flexibility in situations of continuous health monitoring (ARM) at home and with mobility, generally speaking, in ambient assisted living (AAL) environments. 
These monitoring systems are also able to assist all kind of people with different levels of intellectual and physical abilities and/or disabilities. For instance, this system is able to help a firefighter during a fire situation by monitoring his vital signs. Also it is capable of assisting a patient or a pregnant woman in an ambulance on his/her way to the hospital allowing the physicians to know ahead all the relevant data [6] [7]. This prototype engages in such a way that a handler can have accurate information about a patient, a child or an elderly who can be lost, astray or have his vital signs altered and that a SOS call is in order. It is also possible to alert the elderly about a mandatory consultation.

These technologies have had a tremendous development in recent years and they play a very important and active role helping in the surveillance of the elderly. These solutions allow people to have an almost problem free life by encouraging them to have their independence. Moreover, they can offer great individual levels of independence, comfort, and active involvement in the community in a secure way thanks to effective caring and continuous health monitoring and location.

With the establishment of multi-agent systems it is possible to deploy new assistive technologies [8]. This dissertation presents a solution created to provide health monitoring in AAL environments with mobility support. This proposal uses a multi-agent system with self-configured interventions. The considered mobile healthcare enables the identification of possible hazard situations to diagnose diseases. Moreover, such system can offer preventive, diagnostic and therapeutic benefits. Furthermore, whenever possible, it makes a readjustment of long-term medical treatment based on the analysis of previous stored data, and also applies medical knowledge to recognize, manage, and rehabilitate injuries related to sport activities.

This mobile application can still perform an outdoor intervention in circumstances of possible accidents or risky situations, reducing the intervention time of medical institutions. The main objective is to allow time reducing from the beginning of symptoms till the admission at a 
hospital. The relationship between mortality and time delay on treatment is a crucial factor, because the continuous monitoring of bio-signals promises to improve traditional healthcare in many ways. The stored health data and the gathered longitudinal data offer health care providers a more complete understanding of their patients' health and the monitored real time data of the elderly allow an historical analysis of the collected parameters as well as an immediate response in emergency situations in which the elderly cannot react, or provide feedback, to help them modify their behavior.

This proposal can be embedded on a mobile device, such as a smartphone or a tablet, with a supplement of body sensors, with sensors already included in the mobile device. This proposal has a fall detection solution a geographical localization and a biofeedback monitoring activity and also bio-signals in real time with mobility support. On the other hand, still has the possibility to collect data in an offline context in order to follow a critical patient.

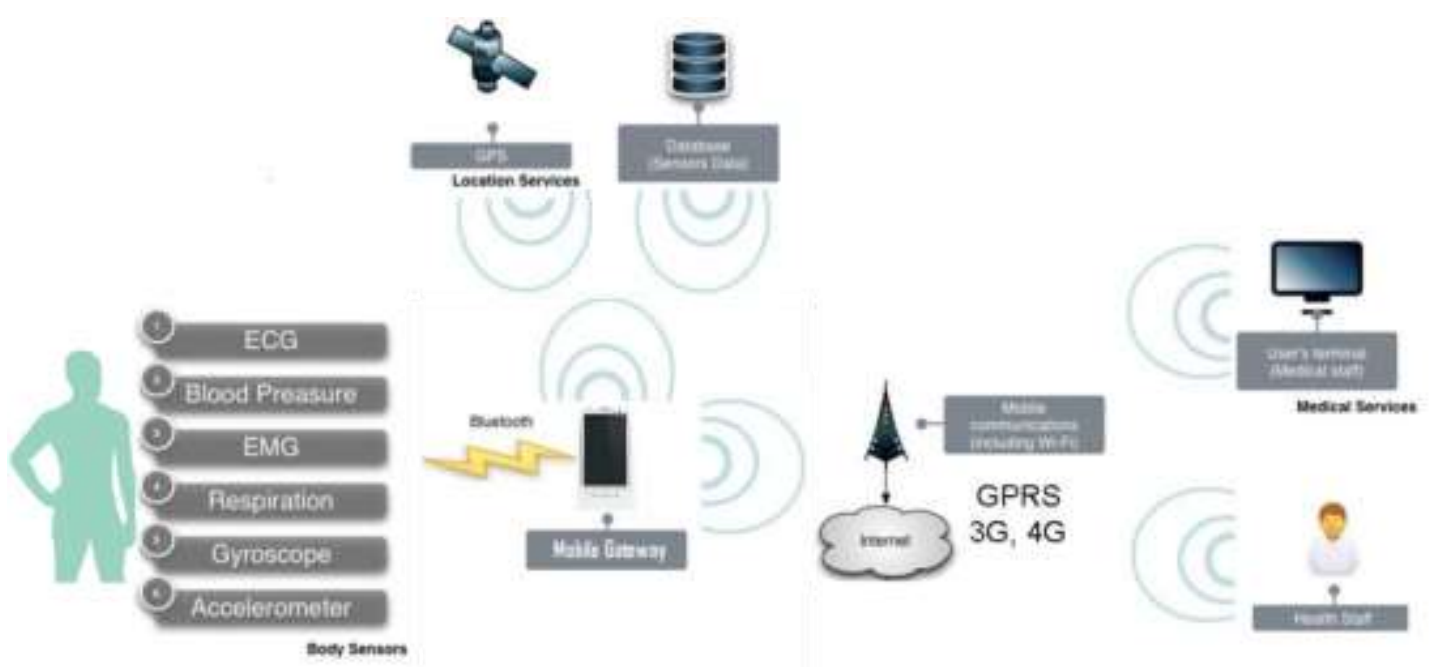

Figure 1 illustrates typical mobile health systems, containing one or more monitoring and/or surveillance resources and devices, such as wearable sensors. They should be connected to the Internet to allow information sending, data storage and retrieval.

Therefore it is important to remember that today, we have more and more systems that are ubiquitous and mobile solutions using technology 
have to be unobtrusive, affordable and reliable. The AAL proposed solution allows the use of a simple phone as a device something, common in daily life for young people but also for the elderly. This way you can easily be a "bridge" to communicate abnormal situations even with a complicated schedule. In dangerous situations it is exclusively vital for the elderly who tend to be afraid of the use of new technologies.

\subsection{Problem Definition}

Regardless of our age we all get sick and we can have bad existing changes in our bio-signals. The older we are the higher the likelihood of getting sick. This probability increases proportionally with your age. The advanced age of people with illnesses can lead to death because their defenses are more difficult to recover. Given the increasing of elderly people around the world, there are new challenges to the improve quality of life for these senior citizens, so they can live longer and with the best quality of life as possible.

Thus it is expected that this may be the ideal situation to prevent a consistently stressing healthcare systems in Europe for an increasing number of elderly and chronically ill patients. This way it intends to become a viable solution, for example in home healthcare monitoring systems. The eCAALYX project, it is a manageable, expandable, interoperable and low-cost Ambient Assisted Living (AAL) solution. The eCAALYX Home Gateway relies in the use of open standardized protocols, as well as a modular architecture in order to create an unifying layer between sensors. This platform provides the use of various types of sensors like accelerometers to detect current activity, such as falls [9].

Well, towards a real problem in modern society, is common in developed countries to demonstrate the seriousness of the problem. Therefore, Mobile Healthcare Solution for Ambient Assisted Living Environments may prevent or even avoid dangerous situations and lower death rates. 


\subsection{Objectives}

This dissertation focuses on the design, construction, deployment, and performance evaluation of an AAL solutions for a fall detection solution with geographical localization, biofeedback monitoring activity and biosignals data in real time with mobility support environments. This solution considers two mobile applications: one prepared to personal computers using Java and another for an Android mobile platform with falls detection and biofeedback monitoring, in offline data storage or in real time both for elderly and weaken users. This proposal is about the construction of a consistent and robust solution for mobile health environments, using a simple system and adaptive proposal. The system will provide biofeedback information through the internet. It also features audio notifications to users and outside alerts, by sending a short message service (SMS) or electronic mail (email) to a caretaker with a brief description of the health problem and GPS coordinates the user location.

These main objectives are accomplished, and the following intermediate objectives are identified:

- Study of state-of-the-art in Mobile wearable sensors, and Ambient Assisted Living, approaches and further representations of the challenges, as well as the study of biofeedback acquisition data, and techniques to acquire inertial and bio-signals methodologies;

- Research by leading mobile biofeedback devices available on the market, comparison among them and the analysis of the system requirements for two solutions presented;

- Proposal and deployment of an AAL solution for mobile environments, including a static APP for Java to desktop and a 
mobile APP on Android OS based on detailed requirement analysis system;

- System demonstration, performance evaluation and their validation on several mobile devices and with multiple sensor systems in proposed solutions using real prototypes.

This research expects to produce two distinct applications, and it will also provide the chance to disseminate knowledge making significant contributions to worldwide scientific entities.

\subsection{Main Contributions}

This section presents the scientific contribution of this dissertation to the state-of-the-art mobile healthcare solutions and the AAL environments.

This contribution presents two robust and adaptive solutions to elderly people. There is health control by accompanying a patient because it is possible to establish a more effective diagnosis allowing improving their treatment. The second solution allows the help of elderly that need regular healthcare services and are dependent of physicians. It offers mobility support and includes biofeedback monitoring using body sensors that collect data from the elderly and offers support for remote monitoring. The presented proposals follow elderly people, storing collected data through Bluetooth and allowing an analysis of possible warnings based on the input, and real-time monitoring of key parameters.

A paper with this contribution entitled "A Mobile Healthcare Solution for Ambient Assisted Living Environments", was presented to the 16th International Conference on e-Health Networking, Applications and Services in Healthcom 2014 IEEE, Natal, Brazil, October 15-18, 2014, and the review is completed. 


\subsection{Dissertation Structure}

This dissertation is organized in seven chapters, subdivided into multiple topics.

This Chapter 1 - Introduction: it starts by focusing on the topic under study. It detects some problems in the way this research has been implemented, it defines the most important objectives, and presents the main contributions as well as the dissertation is structure itself.

Chapter 2 - Related Work: it addresses the state-of-the-art, approaching the literature on Mobile wearable sensors, Ambient Assisted Living, including a brief review about health systems, focusing on health sensors and the importance of bio-signal acquisition and their processing. Then the most related and relevant work biofeedback methodologies.

Chapter 3 - Requirement Analysis: its presents the requirements analysis in order to fetch all the system necessities, UML diagrams (behavioural, interaction, and structural diagrams) and used technologies.

Chapter 4 - Devices Used on the Prototype: it introduces and discusses all the devices used on the two prototypes and their relevant technologies, as well as photographs of equipment's used in the prototypes and their technical specifications.

Chapter 5 - AAL Mobile Solution Demonstration: it addresses the considered AAL solution for mobile environments APP. The mobile application is introduced and all the features are discussed. The system architecture is the proposed approaches for biofeedback monitoring, falls detection with outside network communications. 
Chapter 6 - System Validation and Performance Evaluation: it described the results of the m-Health application used on the tests, and focuses on the performance validation and evaluation of the solution.

Finally, Chapter 7 - Conclusions and Future Work: it reviews in an argumentative way, all the work developed along this dissertation, considering the conclusions of this work and challenges for future developments. 



\section{Related Work}

In order to focus, it is important to present some related work about the matter on this dissertation. An important reference to bear in mind is the existence of lots of information about some subjects, and sometimes the very latest information is obsolete, because progress is so quick in this field. However, this gives an excellent point of view and leads to development of new techniques and solutions. From the basic health care systems to the next level on modern mobile health this is a significant start to implement this mobile solution.

This chapter presents the related literature to AAL solutions in Section 2.1 it is presented one way of implementation anywhere, to use it anytime and finally used by anyone. In Section 2.2, approaching the different topics about Security, privacy and confidentiality of information in Ambient Assisted Living. Section 2.3 discusses some health care technologies. Section 2.4 presents some available health sensors systems, enumerating and describing the most used in the mobile application. Section 2.4 approaches a general scope in falls detection, biofeedback monitoring works and some types of Health systems as so as the m-Health and applications for common users found in the literature. Finally, this chapter is summarized in Section 2.6. 


\subsection{Anywhere, Anytime for Anyone.}

In most of these projects, prototypes are built to achieve independence, well-being, and in general, good quality of life and conveniences beyond the limitation of spatial and time constraints.

We can also include assistance to carry out daily activities, health and activity monitoring, enhancing safety, getting access to social, medical and emergency systems, and facilitating social contacts.

Speaking of systems has been well implemented and respecting all safety regulations and privacy of data without forgetting the acceptance by users and caregivers (described below).

People who will take this type of assistance probably suffer from impairments, disabilities chronic illness and mild cognitive impairments or chronic diseases, (e.g. hypertension, cardiovascular diseases like tachycardias are the main cardiovascular diseases and these can be diagnosed by analyzing the changes in ECG pattern). According the World Health Organization, cardiovascular diseases are the number one cause of death worldwide, responsible for an estimated 17.1 million deaths in 2004 (i.e., 29\% of all deaths worldwide) and economic fallout in billions [10].

But also bone diseases, depression, obesity, physical inactivity, smoking, people with asthma, diabetes diseases or chronic heart failures like heart attack, sudden cardiac death, aneurysms, and immune disorders are widely believed to be related pervasive healthcare systems help to minimize hospital stays and in doing so enable an independent life in a domestic environment [11].

There are consequences that are not so straight but nevertheless are people who have to deal with in their daily life with these problems, which can be psychologically exhausting such as a nurse [12]. Or in more permanent situations such as blindness or loss of one arm, leg, which does not necessarily require a getting to a hospital or clinic regularly. This represents a new life concept impaired individuals not as different people but as people with different capacities. Being implemented sensor systems 
in daily usage scenarios as shown in [13] and [14], but without forgetting that the AAL should easily integrate novel solutions and equipment's facing a changing context.

But the elderly cannot always stay home, sometimes it is necessary to leave the house for a walk, a journey to the doctor, or even an urgency, these cases can also find AAL technologies that enable the monitoring of an elderly throughout his/her visit to a hospital or care facility, so there is a full and constant updating of his/her main vital signs informing doctors or nurses of what is happening so they can be prepared a quickly and effectively. Especially in situations when the elderly has a system which has been useful before, during, and after a cardiac arrest for continuous monitoring of a patient.

Nowadays we want our elderly to be more time in their homes so they do not feel intimidated by the constant presence of a doctor, nurse or even in company of other strangers every day. By avoiding these situations it is possible to provide more efficient utilization of physicians, reduced hospital stays, shorten the frequency of visits of home-care specialists, and hospital readmission rates. Therefore it can easily contribute in the reduction of death due to heart attack and other cardiovascular diseases, also it can be used for providing health service by specialized doctors, mostly on rural or remote areas. Also promoting health education contributes to reduced healthcare costs [15].

\subsection{Security, Privacy and Confidentiality of Information in Ambient Assisted Living}

Currently there are effective and adequate ways to predict the actions, behaviors, habits, thoughts, dangerous situations, intentions, emotions and health conditions to ensure quality of life in people with cognitive or physical difficulties allowing them more autonomy following remotely without being enclosed in a hospital, health center, private clinic, 
or nursing home. People maybe like the comfort of their home or even outside. It is possible to provide greater promotion on mobility for users' in order to be outside their home, through accessibility to their working environment, leisure, health and wellness.

The AAL aims to create better conditions of life for the older adults and this technology can provide amazing contributions to healthcare through mobile services and solutions for the patients and elderly [16].

It can develop advanced products, services and systems to people with major physical limitations. It is evident that the topic is a problem, not only in a social level, but also at the level of medical treatment. This way, the AAL Services are build up on PERceptive Spaces prOmoting iNdependent Aging (PERSONA) distributed software platform and therefore benefits from its wide range of functionalities [17] [18]. It supports elderly people outside their home and this way provides information and assistance near public buildings and inside public buildings. The Persona, is the most advanced architecture approach of our investigation, because it provides flexible, intelligent, ubiquitous, context-awareness, distributed and secure AAL Services.

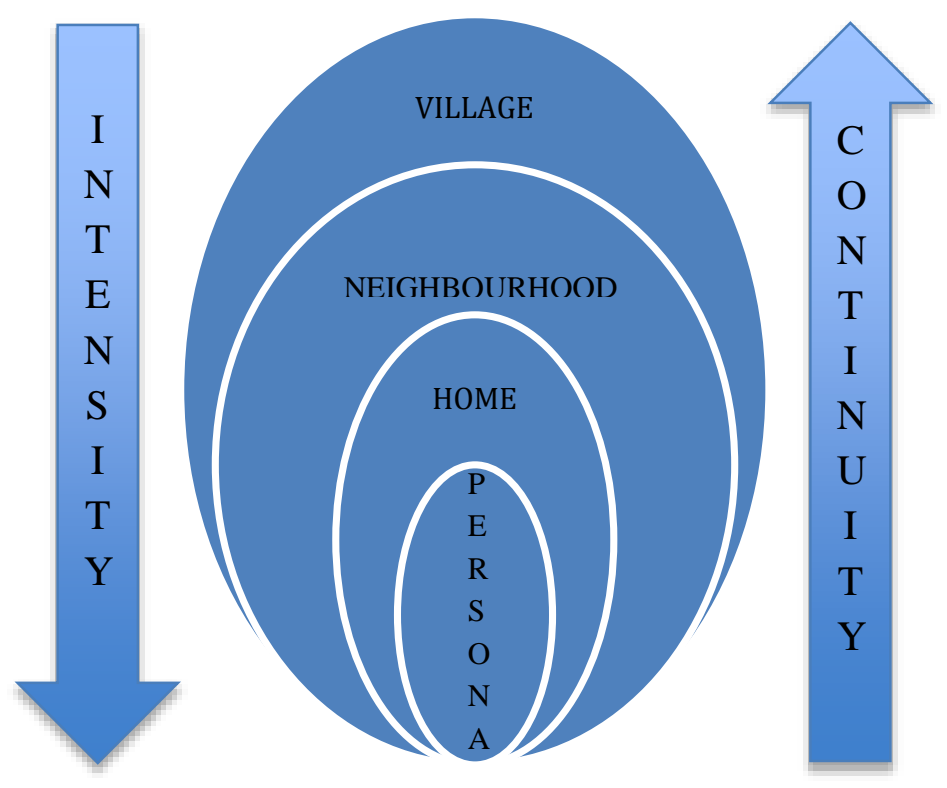

Figure 2 - Illustration of range (continuity and/or intensity) of PERSONA platform. 
Persona project's software allows the creation of devices that will help the elderly, still facilitating the improvement through the innovation adoption of smart homes for elderly. These innovations are always an advantage because using simple objects such as a television can easily be turned into an object of assistance to the elderly.

This object can further comprise similar situations with regard groups of functionalities that can be implemented, for example, as a nutritional helper [19]. Therefore it could aim at developing a scalable open standard technological platform for building a broad range of Ambient Assisted Living Services.

These types of communication services to the "world" must be applied carefully in order to maintain the privacy and ubiquity of the user. It is intended these technologies may have as one of their goals to allow total privacy, and may use strategies to hide the information owner via anonymization or the use of pseudonyms. For example, the video cameras surveillance is becoming increasingly common but we know its purpose. There is a notion that there is some privacy when using those images, which are used in closed circuits but most of time they are not visualized by anyone, being used only in extreme situations such as should evidence for a possible assault.

\subsubsection{Direct Advantages and Disadvantages of AAL}

When one gets older, it becomes more difficult to take care of himself and AAL allows the person to stay at home with all the necessary support and not needing to visit a nursing home or hospital. How the elderly are not resorted to medical care will allow instant money saving in health care. Faced with this independence and comfort friends and family can help seniors. So with this monitoring, elderly people can carry out various activities in the comfort of their house without having their privacy invaded or even are been followed during administering medications. 
Moreover, recent advances in image processing and pattern recognition techniques enable the automation of many surveillance tasks, increasing the risks of privacy infringements, which are an even more vulnerable result of globalization. Those protection aspects raise some severe concerns regarding the protection of our civil rights and if system architectures respect and protect privacy. So this type of assistance may not be the best choice for every senior, but when living in nursing homes people tend to require more hospitalizations and doctor visits. This factor can also be a barrier because the elderly is in an unknown environment, being afraid of isolation from family and friends and is more likely to suffer from depression due to his/her lack of Independence.

Maybe the major disadvantage is the difficulty for elder people to interact with new technologies, because they have many difficulties to understand and learn how to use them, sometimes it is a very arduous path [20].

\subsubsection{Privacy and Confidentiality}

In [21], Kofod-Petersen raises important things about the elderly as for trying to avoid issues of privacy and confidentiality. Q. Jones and A. Grandhi et a [22] present P3 systems that enable a new class of locationaware information, which revels one interesting problem in the context of ambient assisted living ( $A A L)$, because for the typically elder it is expected to share, not only location information but also biometric data, such as blood pressure.

To Martina Ziefle, Carsten Rocker extends those individual factors (gender, age, health status) as being an important influence to adapt to this decision. Given their study it was verified that the regular use of existing knowledge and technology to provide preference of mobile devices and Medical technology, integrated into home environments, seems to be more critically evaluated than smart clothing or mobile devices [11], 
because technology crosses the limits of social acceptability and fashion of commercial applications [23].

The dilemma posed concerns between the Benefits vs. Privacy, but the health care sector is strictly regulated, particularly regarding information privacy and confidentiality.

\subsubsection{Security, Safety and Comfort}

The implemented systems do not provide any security about data transmission or authentication methods. The topic is not even discussed in most of the analyzed papers.

Security is a burden that exists in order to ensure privacy and confidentiality as well as the necessity of being careful with the use / manipulation of data as one of the biggest questions for the concept Ambient Assisted Living. Although Transport Layer Security is a widely spread and has a high-performance protocol it does not completely suit the demands of embedded device, but it is well-adopted in a general way. It has been seen that is capable to securely interconnect or Web Service Security [24].

Farzad [25], presents one solution when everybody else may forget achievement of the activities, or realize actions of daily living incorrectly, and by this way enter dangerous situations. A smart home project is proposed as an ambient intelligent environment, allowing the resident to be observed continuously through the embedded sensors, and this continuous surveillance permits the user to be assisted automatically through the embedded electronically well-regulated actuators. In this work, this proposed approaches to understand the sensors observations and automatically reasons in the vital assistance. Or Sohlberg [26] discusses the limitations of adults with cognitive deficiencies in terms of navigation, and the importance of establishing policies for daily routines and supported methods during walking trips, for example. Obviously, the use of all these technologies in such systems is extremely important for their acceptance. 
Thereby there must be some concern and interest in development of interfaces that will support natural forms of interaction.

\subsubsection{Reviews}

Given these type of systems we also have issues of complexity because these systems raise fundamental questions of behavior, communication and technology acceptance but this system is implemented as a personal trusted agent and supports privacy decisions of a single user [11] [27]. Given the generalization, these type of implementations used will become more accepted by our society, without forgetting that these limitations affect their conceptual/Physiological, social and practical skills, which can have a substantial emotional and behavioural impact on their lives and surrounding environment [28]. Thus to Hervás, Bravo and Fontecha, the system moves through a series of familiar location points in guiding the user to a given destination. The navigation information can be displayed on the screen in an augmented reality view, showing the locations of landmarks along the chosen path in addition to contextual information related to the user, such as locations of relatives and tasks that must be performed at specified places. Therefore the development of new technologies provides mechanisms to improve the personal autonomy of people with cognitive impairments and help relatives to monitor their states by tracking their trips and being aware of their daily tasks in a specific environment [29].

In AAL scenarios exit several barriers including lack of access to infrastructure, lack of convincing data on effectiveness of recorded for many type of sensors and these purposes, costs, healthcare provider resistance as well as privacy, legal, security and ethical issues. Because this data can be assigned to certain persons and this way the date may contain important information to hackers or even health insurance companies and may avoid involvement in tele-health interventions. Thus, this data needs special care regarding privacy and security allowing to the AAL platforms while remote backup and restore functionality can be provided but can 18 
have a major impact on one emergency situation that can have potentially fatal consequences [30].

\subsection{Analysis in Healthcare Technologies}

All these technologies should have as its primary objective, the definition of rules that everyone can follow, allowing it to be possible to implement these technologies, thus creating standardization. For example, the Continua Health Alliance [31] makes an effort to spread these standards in different associations to use open standards and frameworks to enable a simpler and more open software architecture for applications in the field of AAL. Given the homogenization is possible to take into the future interoperability and plug-and-play solutions as one great achievement. But currently there are ongoing developments especially in the open source community allowing early integration of these technologies to facilitate important requirements like security, modularity and expandability.

To support $\mathrm{AAL}$, different location and tracking technologies are being exploited in many AAL systems. Technologies like WiFi [32], Global Positioning Systems (GPS), platforms [33], ultrasound devices, Camerabased, Infrared Radiation (IR), Bluetooth, and RFID Radio Frequency IDentification [34] have been widely used for tracking people inside/outside houses. Most of these systems use some sort of wearable devices such as wearable tags, watches, bracelets, or sensors like video cameras, and motion sensors to detect peoples' locations or in integrated into advanced AAL systems, such as wearable sensors to monitor vital signs, or teleconferencing to combat loneliness. Even through the existing location and tracking services are considered a great step-forward for providing location knowledge, they are not quite adequate for AAL.

The ZigBee [14] [32] is a well-known wireless communication standards architecture and is made up by a set of blocks called layers. Each layer performs a specific set of services for the layer above. On top of this 
the ZigBee standard defines the Network (NWK) layer and the application (APL) layer. They are one of the most popular transmission technologies like Bluetooth or IEEE 802.15.4 wireless technology hare the main features are low complexity, low cost, low power consumption, low data rate transmissions, to be supported by cheap, fixed or moving devices and sometimes losing the acquisition performance to improve the duration of collection of data.

Moreover, there are also a few proprietary solutions, including those designed specifically for biomedical monitoring applications and many more protocols that are used like as BACnet, KNX, 6LowPAN [35].

\subsubsection{Assistive Technologies and Electronic Services in AAL environments}

Since the term AAL comprises new concepts, technical products, services, logistics, new technologies and social environment, it is possible to distinguish between several levels of observation and various stages of development, including society in some of these potential mechanisms that are already found on the market. Therefore, the first generation is the basic social alarm that can be triggered to raise an alert in a telecare center which allows the call operator and service user to talk one another and decide on appropriate actions. The second generation includes devices such as heat sensors and flood detectors which have a passive or automatic alarm dimension and can trigger alerts to the telecare center without human intervention. The third generation of telecare is able to monitor everyday activities of the service user through motion detectors and door sensors as well as set parameters [36]. Breaching these, leads to interventions by the call operator in order to assess the situation, and then it's able to diagnosing the problem and determining appropriate action. The fourth generation deals with web-based communication platforms in order to integrate different technological systems and devices as well as different services [37] [38]. The fifth generation integrates robotic devices 
in the telecare process, like the first prototypes can be connected to social alarms (GiraffPlus) [28].

In all this processes there is always the need of communication services where this goal is to help older people who become less mobile, because meeting other people outside becomes more and more complicated if not impossible. Sometimes they are the bridge to medical services intending to focus on the detection and prevention of emergency situations, in a long-term treatment of chronic diseases in elderly without mobility, and also prevention and early detection of illnesses.

There is also a substantial convenience services research on AAL like for example, a few sensors for supporting users in everyday activities by compensating individual physical disabilities. This sensors used to achieve human activity usually have low power consumption and cheap implementation costs, such as wearable-based like accelerometers, gyroscopes, or ambient-based like video cameras, thermal and other kinds of sensors.

\subsubsection{Smart Artefacts and Mobile Devices}

Perhaps they are the easiest things to implement, because nowadays we have all have mobile phones or PDA's, that can show our location. It might also be a device that has similar characteristics regarding technology-enhanced everyday objects, which is equipped with sensors, memory and communication capabilities (see, e.g., [39] or [40]) and which is able to capture information about its surroundings, communicate with each other and react accordingly to previously defined rules through internet, and social networks. All these objects are capable of picking up and transmitting the information so as to allow it to be displayed on mobile phone or PDAs, so it's essential to take full advantage about their features and capabilities.

Internet has made it possible to create more complex applications such as virtual worlds (VWs). VWs are 3D simulations of an earth-like world 
where users are represented by a graphical representation of a human being. VWs is able to endorse online social interaction with objects, or with other users and interact in a way by means of text chat, voice or even video [41]. This type of VWs is common like Second Life [42], but is more important to focus in health care, like Second Health [43]. Here the concept of Internet of the Things (IoT), is considered one of the greatest advances in communications in recent years, because it is able to integrate in Internet the sensors and objects located that surround us and provide foundation for the development of autonomous applications and services, like instant diagnosis of health incidents through the Cloud infrastructure [44] [45].

\subsubsection{Wearables Sensors and/or Embedded Mobile Device Sensors}

Wearable computing technologies, as originally presented by Mann in 1996, and these machines are no longer separated from the persons using them. Instead, they would become an unobtrusive extension of our very bodies and long-term home and outdoor health monitoring and communication services for elderly people, so that their health and functional capability can be maintained for a long time, and an enhanced lifestyle can be promoted.

All wearables have to have minimizing impact of the system for the patient is one of our main concerns, sensor devices have to become smaller, more powerful and be energetically in order to be chosen to be wearable, non-invasive and of minimum impact to the user. They will all have wireless communication capability and batteries [46]. When we make reference to these sensors some are more accepted outside of the laboratory context because they can easily be used in everyday life, because they are small, lightweight, with long lifetime and power autonomy and enough comfortable to use. 
This concept of 'wearable computing' envisions computers to an integral part of our everyday clothing [47], like e-textiles (BIOTEX) to integrate sensors in textiles [48], Fingerings [49], pendants [50], belts [51], gloves [52], smart shoe systems [53]. Those projects have adapted the traditional home environment utilizing ambient, unobtrusive, and nonbody-mounted sensors and microsystem technologies, in order to detect irregularities of elderly people in their daily routines, recognize fall accidents, and reason on acute disorders in health condition. Projects like, NOCTURNAL (Night Optimised Care Technology for UseRs Needing Assisted Lifestyles) system which provides night-time support for people with dementia [54], or a system to monitor children [55], the AAL@Home [56], and to improve posture, avoid developing pressure ulcers and ergonomics issues [57].

\subsubsection{Smart Environments and Smart Homes (SHs)}

The appearance of Internet and Remote Services has brought even more possibilities to complement the indoor functionalities of consumer devices with the advantages of otherwise costly professional systems and services on remote.

This "invisible" technology represents a challenge, because it needs to accomplish several requirements, like People identification, and localization, data protection, dignity as well as medical demands, an experimental space is necessary, which enables to study patient's life at home.

The "SMART" should be able to easily intervene in risky situations even anticipating potential accidents and take precautions if necessary. There are several studies with several features that could be implemented into smart homes, like the recognition of audio or voice commands, location or with the introduction of sensors on doors, beds, couches or motion sensors and of course without forgetting the installation of cameras in order to enable the identification of daily activities of the inhabitants of 
the house helping them in a exercise program measuring the contact forces applied to the ground by the user's feet [58] or to cook a delightful meal [59], because it encourages independence and the maintenance of good health, for example through an intelligent medicament blister helps to increase a patient's compliance and the task-based calendar assists people with cognitive disabilities in their activities of daily living [60].

In an extreme situation it is possible to implement systems of integrating building networks and body sensor networks [61] or a smart city like the big academic and industrial such as Smarter City [62] of IBM and other companies that have showed increasing interest in this domain.

\subsubsection{Reviews}

Firstly these technologies need to have a set of sensors and actuators in order to allow the monitoring of a person or group of persons, but alone this implementation of sensors will not create Smart Environments.

Faced with these types of systems, their ability to be intelligent and therefore able to provide a set of behaviors of a person. In this case it may be a good indicator of health status to monitor and assist a person. If some type of intervention is necessary, the actuators instilled in their own environment or even make an adjustment at house temperature, alerting any caregiver that your wearer has a very high heart rate or even nonexistent.

The Wearable Sensors are able to effect more efficient evaluation by monitoring the state of a user and with the assistance of Mobile Devices that serve as bridge communication, thus allowing a more efficient way to engage if required. These sensors are of great importance in Aml since they can be handled according to their requirements aid in the activities of its users. The durability and longevity of the batteries are an advantage so those sensors shall be activated only when it is required, otherwise they must keep off [32]. It is always important to establish how well a sensor may be or not critical to a potential dangerous situation. The position 
Baquero [32] is that to adequately handle the various types of sensors and the different sensor communication forms we propose the use of a uniform sensor-handling scheme. This scheme will enable Aml systems, such as those developed with our Modular Scheme for the Integration of Ambient Intelligence Systems, to handle sensors in a uniform way. We can speak about the creation of a smart sensor by combining various sensors, an analog interface circuit, an analog to digital converter, a microcontroller and a communications interface, and also include a power supply in the form of a battery [63]. Without forgetting the system Caalyx (Complete Ambient Assisted Living Experiment) light mobile device that was founded in 2009 and had the participation of six different European countries and this device monitor several vital signs and send the recorded data to the nearest health care center obtaining results for usability, functionality and reliability.

There are also systems called Activity recognition or of the intention recognition that have significant applications in ambient intelligence, for example in assisted living and care of the elderly this system is called AdAPT [64], IntelliCare [65], and context-CARE [66].

In the inCASA project, a broad variety of sensors shall be integrated into the system. These combine health related, home automation, and ambience sensors. It is expected to measure blood pressure, blood oxygen level, body temperature, blood sugar, heart frequency, partial prothrombin time/INR, weight, symptoms scoring, peritoneal dialysis, physiological rehab (back/knee), movement at home, contact (e.g.: door/bed/chair), wrist movement (actigraphy), ambient temperature, ambient humidity, water leaks, gas leaks [67]. One of those aims is to develop a system that will support the aging population and facilitates them to feel well in their own home. The proposed application will monitor biometric data and track environment parameters of elderly users into their own home.

Thus it is intended that a good system is capable of place irrespective of its implementation, has the ability to be feasible and easily adaptable allowing heterogeneity before the implementation of new 
embedded devices, which are integrated and "hidden" into the environment. All this should follow the parameters of Safety Engineering, allowing the test in real situations to be demonstrated and their effectiveness to be certified [68] [69].

\subsection{Health Sensor Systems}

Biofeedback monitoring system might provide information from patient's body through physiological and inertial sensors. This measurement in a patient can allow the observation of many biological processes and these processes permit an idea of what is happening in a precise instant.

This section will focus on a briefly description of some health sensors properties.

\subsubsection{Electrocardiography (ECG)}

Electrocardiography is the recording of the electrical activity of the heart, and consists in attaching three sensors to the patient's body. Those sensors will interpretate the electrical activity of the heart over a period of time. It is crucial to attach the electrodes to the surface of the skin (and in a different section of it), to record or display the heartbeat through an external device to display in a two dimensional graph known as electrocardiogram. The visualization of that data it is known by five peaks labelled with successive letters of the alphabet $P, Q, R, S, T$, and $U$ as shown in Figure 3. It is extremely important that the electrodes are placed correctly, as known to Einthoven's triangle [70]. 


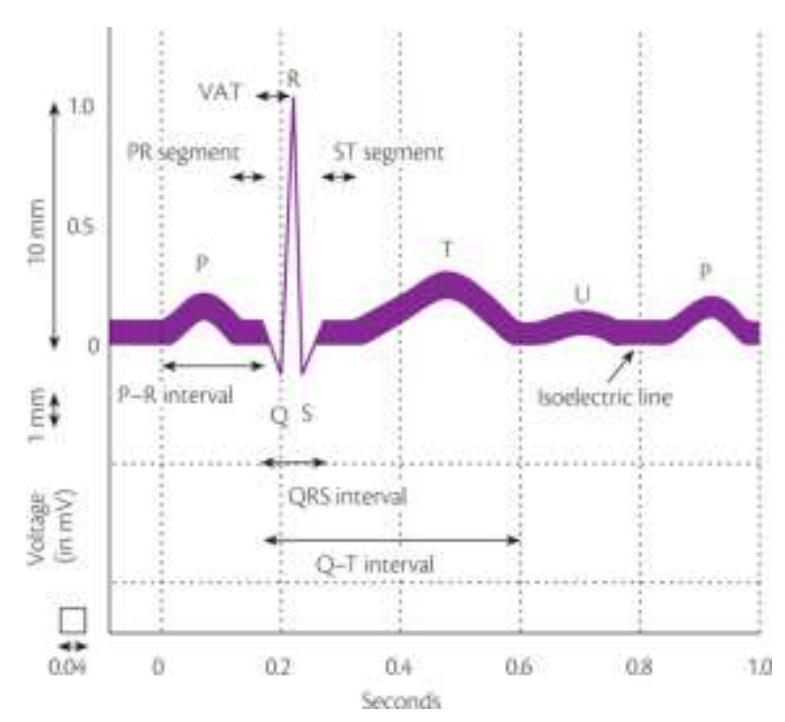

Figure 3 - Illustration of ECG signal detection.

To understand how and what an ECG records, it is important to have a basic knowledge of how the heart works, to identify potential abnormal changes, focuses on the segments and gaps. Several detection algorithms allow us to find heart diseases and other interrelated problems [71], [72].

\subsubsection{Electromyography (EMG)}

Electromyography is a technique to evaluating and record the electrical activity produced by skeletal muscles cells, when neurologically or electrically activated. The instrument called electromyograph produces a record called an electromyogram, and through it is possible to detect medical abnormalities like neuromuscular diseases and also the muscular activity, among others. Those muscles were crucial to maintain equilibrium during movement and posture AAL mobile system is used to falls prevention and detection [73]. 


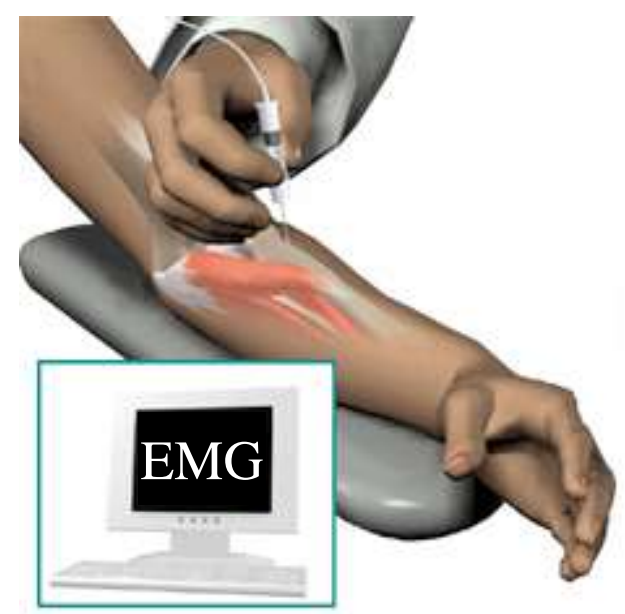

Figure 4 - Illustration of electromyography test.

\subsubsection{Galvanic Skin Response (GSR) or Electro Dermal Activity (EDA)}

This sensor monitors skin resistance and it is directly correlated to the sympathetic nervous system activity of moisture on the skin through sweat glands. This two electrodes sensor' is able to measure the electrical conductivity and it is the means to measure stress or anxiety in situations where these feelings cannot be communicated verbally or socially.

The GSR activity has also been shown as having a correlation with other disorders and activities that involve the sympathetic nervous system, like sleeping disorders and detection of diabetic shock, among others.

Although GRS sensors requires relatively low power and the circuitry is relatively simple it is ideal to use as a mobile device and usually measure from the fingertips, but can also be collected from the wrists or ankles [74]. 


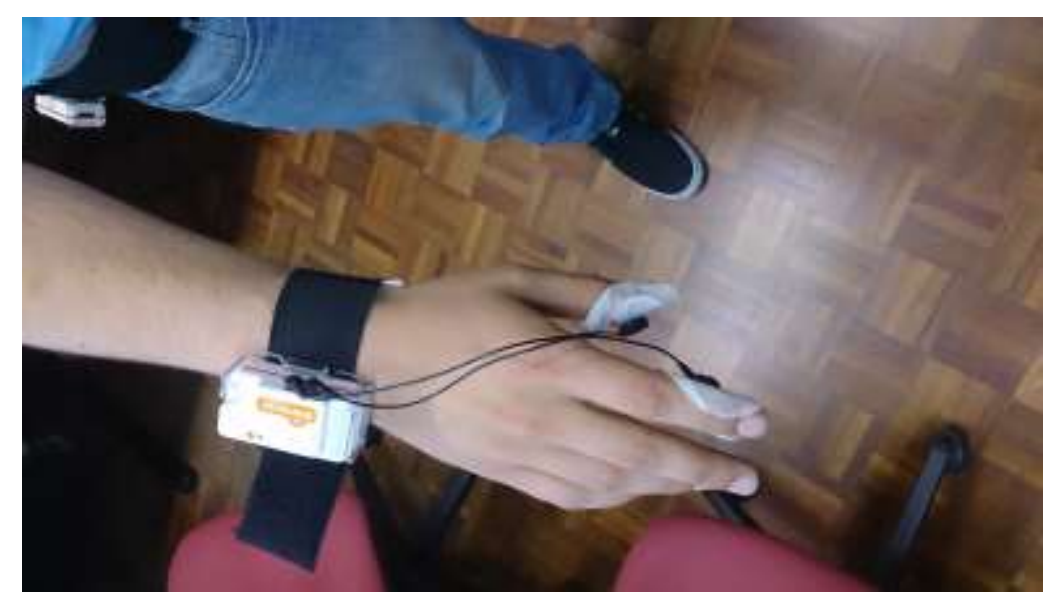

Figure 5 - Illustration of an EDA or GSR Sensor.

\subsubsection{Accelerometer}

An accelerometer is a device that measures proper acceleration ("gforce"). This proper acceleration is presented today in numerous devices, and it is common in mobile devices. When accelerometers are in free fall orbiting and accelerating due to the gravity of Earth will measure zero with the disposal of reading errors. Figure 6 presents a three axes accelerometer module.

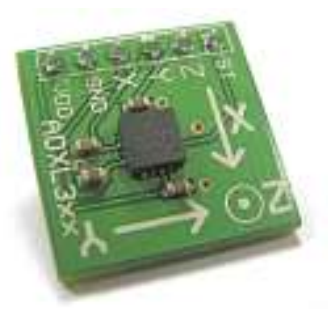

Figure 6 -Accelerometer sensor.

The measure unit is given in G's, which originates from the values at each moment for the three axes presented in accelerometer, respectively $X, Y$ and $Z$. of this sensor to enable identification of the relative position to the ground for a correction of body posture and the accelerometer values to identify situations of potential fall of a patient [75]. 


\subsection{Falls Detection and Prevention Approaches}

Wearable and embedded body sensors collect biofeedback information for the human being. The great variety of sensors and the different ways that they can be applied on persons have contributed to several approaches regarding elderly people monitoring. These approaches mentioned in the literature use several techniques and technologies to capture biometric signals. Thereby, improving the elderly quality of life through several AAL solutions.

Horta et al. presented a solution for fall detection in elderly people [76]. Falls can origin injuries that may cause a great dependence and even death in extreme cases. This system aims to prevent falls and advice the patient or even give instructions to treat an abnormal condition to reduce the risk of falling. Thus, this fall prevention system works in real time and the algorithms analyses bio-signals to thereby warn the user. Monitoring and processing data from sensors is performed by a smartphone that, in danger situations, can send eMails or SMSs (short message system) to a caretaker. This solution was validated through a prototype and it is ready for use.

Along with wearable and embedded sensors, a device that collects and saves bio-signals data, for example, during the absence of his caregiver is the Texas Instruments EZ430-Chronos programmable clock [77]. Furthermore, another solution that allows data configuration collecting specific parameters and real time location while communicating with a mobile device is the use of Shimmer sensors technology [78].

This approach, noninvasive patient monitoring and diagnostics are assisted by a flexible sensing platform, which contains integrated peripherals, specific power management hardware, and open software with a library of applications supported, allows several advantages over many other medical research platforms [79].

There was an extensive range of healthcare applications, but the mainly concern it is the health of elderly and incapacitated people. 
The proposed solution gathers contributions from the abovedescribed approaches. Next sections present it in detail.

\subsubsection{Health Systems}

Currently there are many types of health solutions evolved progressive health applications, like u-health (ubiquitous health), that refer to health management and medical services that utilize wired/wireless networking such as remote monitoring and are able to help the patient anyplace and anytime. Or in other hand have the $\mathrm{p}$-health (pervasive health), this paradigm may be defined from two perspectives: i) as the application of pervasive computing technologies for healthcare, and ii) as making healthcare available everywhere, anytime and to anyone removing this way the location, time and other restraints while growing both its coverage and quality [80].

But also exists e-health (electronic health). It is a quite recent term, dating back to at least 1999. This healthcare practice supported by communications allow the interaction between patients and health service providers or professionals and between institution-to-institution through electronic and digital processes using the internet proliferation. This allows a quicker way to communicate and more convenient access to health services. The solution entitled Data Capture and Auto Identification Reference (DACAR) that this platform presents as a Single Point of Contact $(\mathrm{SPoC})$, presents an innovative rule, which allows the secure capture, storage and consumption of sensitive health care data [81]. This way we see an efficiency improvement of health provider's, reducing costs, easy access to information and improved decisions about possible health treatments or analysis on patients or even increasing treatments effectiveness and more as Microsoft HealthVault to form an e-Health platform that extends across all health care domains [82] [83].

Finally, mobile health (m-Health) refers to wireless portable devices capable of transmitting, storing, processing, and retrieving real-time and 
non-real-time data among end. So, $m$-Health presents one practical solution [84].and this way resolve two important types of issues. For example, the project "Health@Home" aims at solving social problems related to the provision of healthcare services for elderly citizens affected by Chronic Heart Failure (CHF), recent surgical or medical procedures. Their efforts on the collection of data from patients during a period of time after initial post-operative release from a hospital or clinic [85] [86].

In developing countries, there are an immense health care professionals and institutions, but it is important think, when deployed to provide healthcare to remote locations in developing countries, must carefully take into account the existing healthcare and communications facilities, but also socio-economic conditions of populations [87] [88].

\subsection{Summary}

This chapter presented the literature review concerning a state of art in Ambient Assisted Living, a theoretical analysis in healthcare technologies, health sensors systems and covered works in falls detection. These technologies, tools, and techniques focus on biomedical data and on an extensive range of healthcare applications to prevent possible situations of sickness. This information enables physicians to analyze the evolution of a patient and those trying to identify situations of stress for a quick intervention in risk situations. They still look at future challenges, in order to give elderly the comfort of their home and happiness of living freely.

Thus, after a brief introduction, Section 2.1 reviews the possibility of implementation anywhere, its use anytime and finally by anyone. Section 2.2 presented Security, privacy and confidentiality of its information in Ambient Assisted Living. Section 2.3 reviewed the health care technologies. Section 2.4 briefly introduced some health sensors systems available, used in mobile applications. Finally, section 2.5 presented a general scope in falls detection, biofeedback monitoring works and some types of Health systems, like $\mathrm{m}$-Health and applications for common users. 


\section{Requirement Analysis}

Nowadays all software development needs meticulous analysis of the requirements and all specifications of the system implementation. So through the requirements analysis, we can fully identify all the characteristics and behaviors of the systems.

To achieve this goal it is important to model the system accordingly to the specifications of Unified Modeling Language (UML) managed by the Object Management Group (OMG). The implementation of these standard methods allows modeling its behavior, architecture and business process [89]. UML is a general-purpose language with semantic notation in the field of software engineering, which is designed to provide a standard way to visualize, specify and build the design of a system, which consists of setup diagrams with textual description of the main actions and procedures.

Therefore we are talking about analysis which is the key process for software development and clear-cut the goals for observe, their characteristics and behaviors. In this chapter we can view some of the major UML diagrams in order to demonstrate the behavior and structure of the system.

Section 3.1 will discuss the system essential requirements, Section 3.2 and 3.3 will present behavioral and structural diagrams to design mobile solution. Section 3.4 will address used technologies. At last, Section 3.5 summarizes the chapter. 


\subsection{Essential Modeling Requirements}

The most vital steps in these analyses are determining essential requirements. If we set boundaries in our features or constraints the requirements that are presented in the future system are defined. But defining crucial requirements can be a challenging task, because they are capable of having a global and complete vision of the future system to completely define these requirements.

Hence, the following essential requirements were defined:

- Android API level should be equal or above 16, which represents $78,3 \%$ of the active Android devices across the globe [90];

- Bluetooth hardware should be existing in each of the small health sensors to connect with the device;

- Wi-Fi or $3 \mathrm{G}$ modules are required to perform the alerts;

- A folder in device is required in order to allow storage of the collected data of the sensors and data management;

- The system must be self-sufficient to manage resources;

- Every task should run in an invisible and clear way to the end user.

In order to create and integrate those mobile solutions, the abovedefined requirements are required.

\subsection{Behavioural Diagrams}

Behavioral diagrams are used to represent system functionalities. Represents what should happen in the modeled system, the use case it and activity diagrams as described further below. 
This Table 1 shows the main factors that affect the systems.

Actors

Process traits

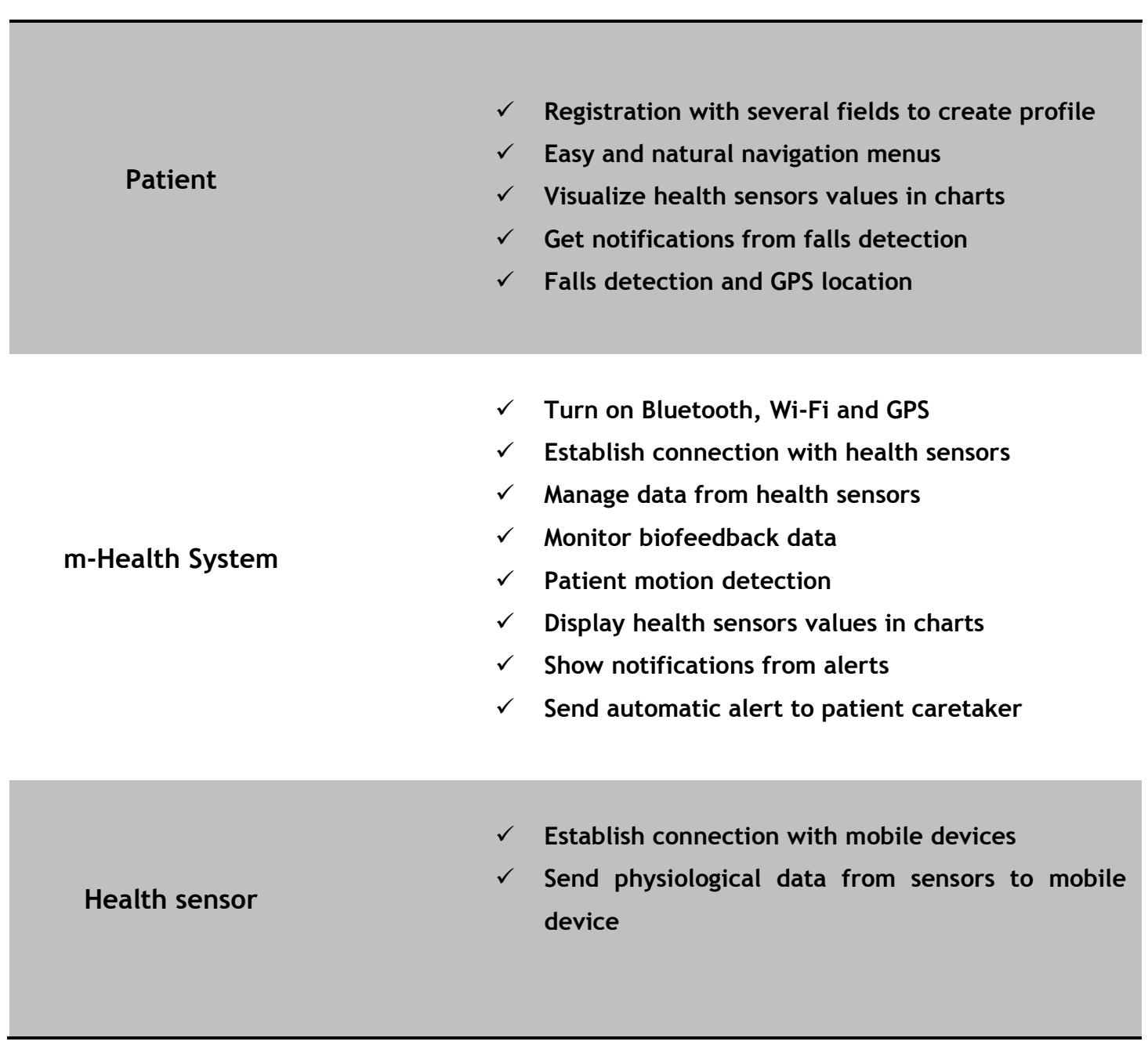

Table 1. Actors, behaviors, and requirements the system must implement.

The main specifications of the mobile solution are here presented. This process was used in software engineering and its goals are divided by various actors to simplify and make a practical implementation.

\subsubsection{Use Case Diagrams}

This type of diagrams can be used to specify the global system functionalities, schematize in a simple way the actions (use cases) that the actors (subjects present in system) should or can perform with the system, 
like simple visualization of graphics, or patient information, for example. The general use case diagram for the $\mathrm{m}$-Health application is presented at Figure 7 for the watch Texas Instruments EZ430-Chronos system and the Figure 8 for the Shimmer Sensors system.

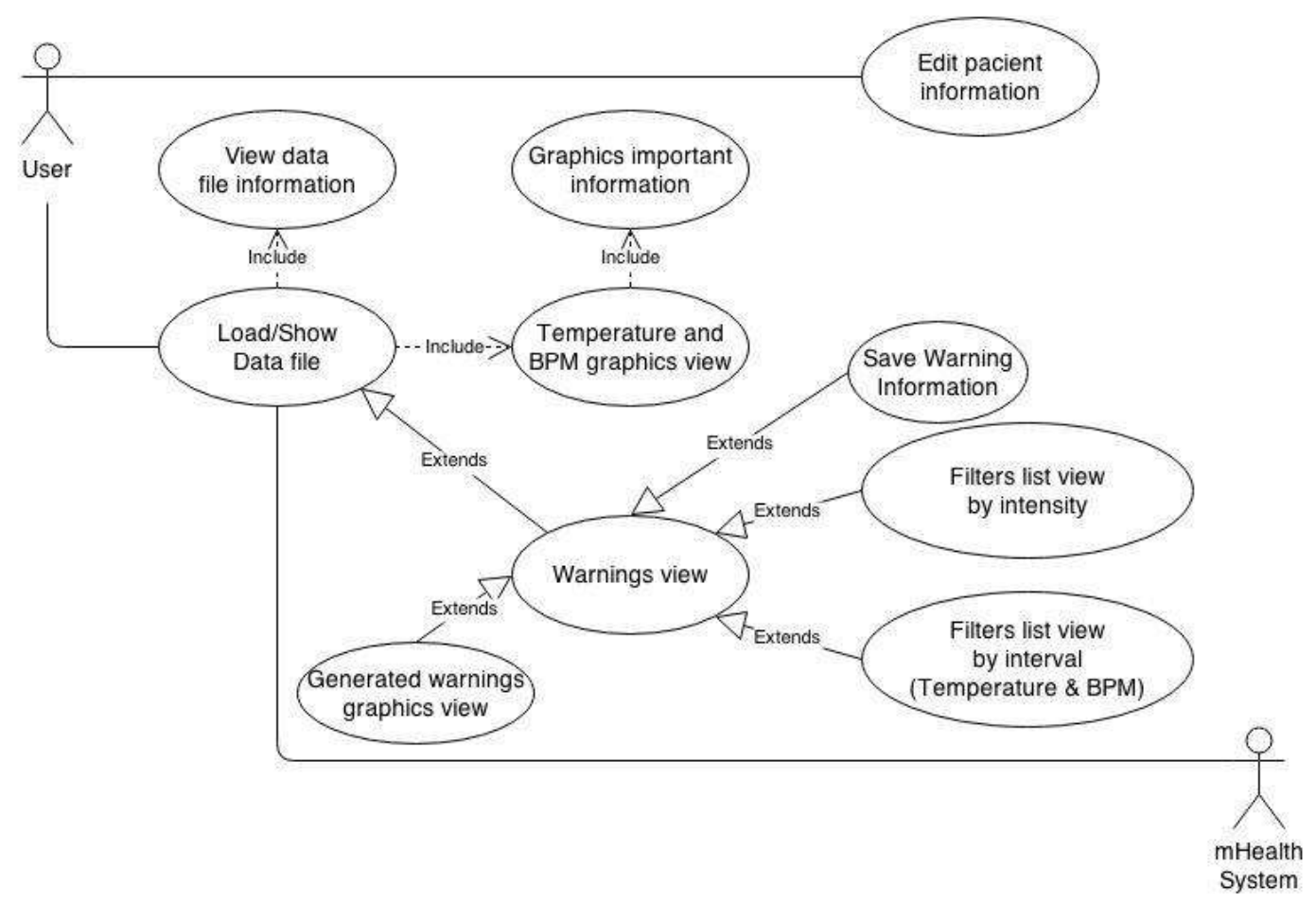

Figure 7 - Application use case diagram for Texas Instruments EZ430-Chronos.

As seen through the use case diagram actors and their associations the most significant use cases actions are presented. The actors showed as a stick in a figure, can be a person, organization or external system. This final structure aims to be easy to use and consists of four sections wherein each has different features. 


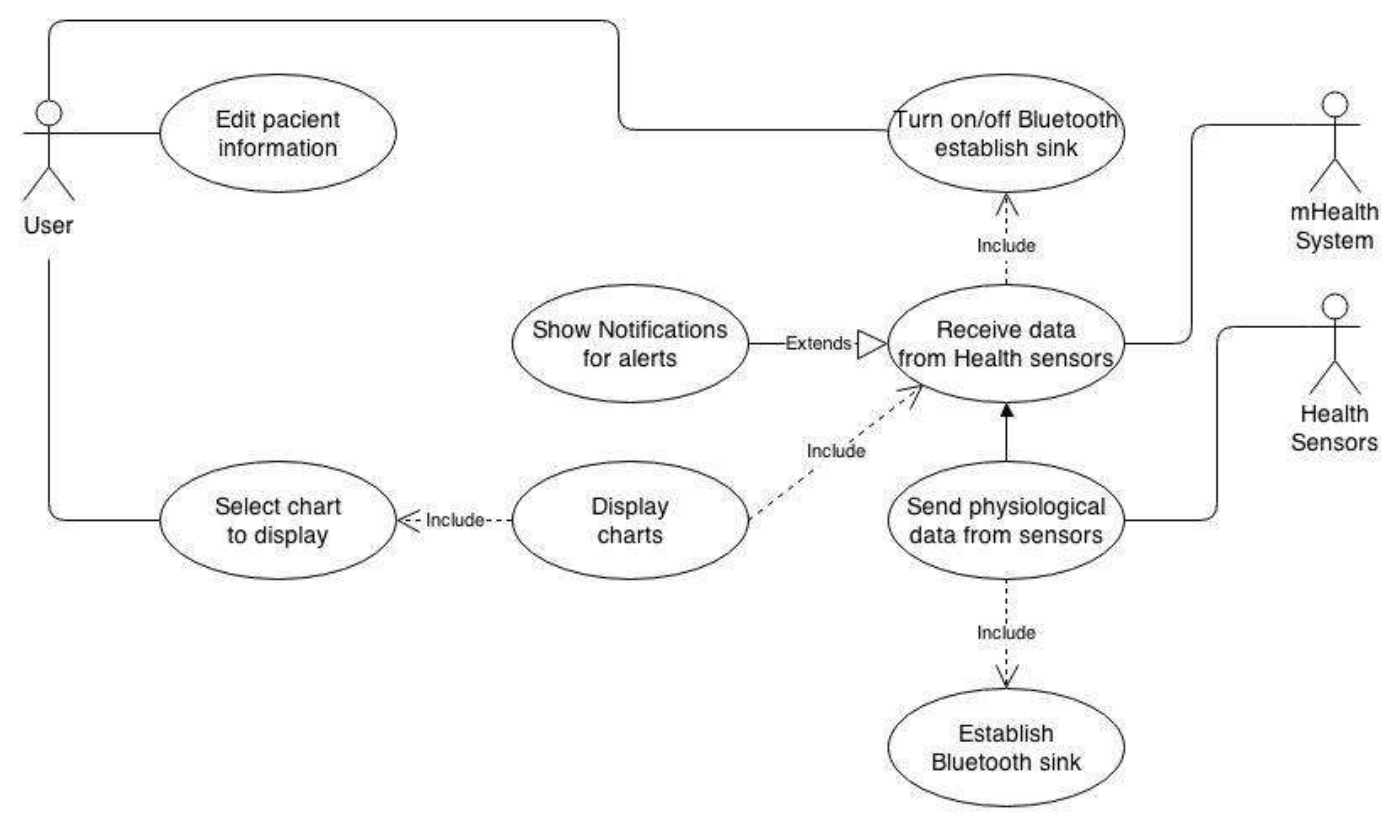

Figure 8 - Application use case diagram for Shimmer Sensors.

Here the use case diagram actors and their associations with the most significant use cases actions are presented. The actor showed in structure aims to be easy to use, and the application has been developed in such a way that to monitor environment the application can run in the background even with turned off screen. Each single functionality keeps running.

\subsubsection{Activity Diagrams}

Activity diagrams are usually used to visualize the system workflow. This different Android activity runs with different sensors and it is presented as a thread and the reading should be measured simultaneously. So the activity diagrams are defined regarding falls detection algorithms, with Notification Alerts, processing and information visualization from sensors and everything is controlled synchronously. 


\subsubsection{ECG and Temperature Prevention and Detection Procedures (Watch)}

The activity diagram presented in Figure 9 represents the bio-signals monitoring solution using several activities and simultaneous tasks and getting several sensor analyses.

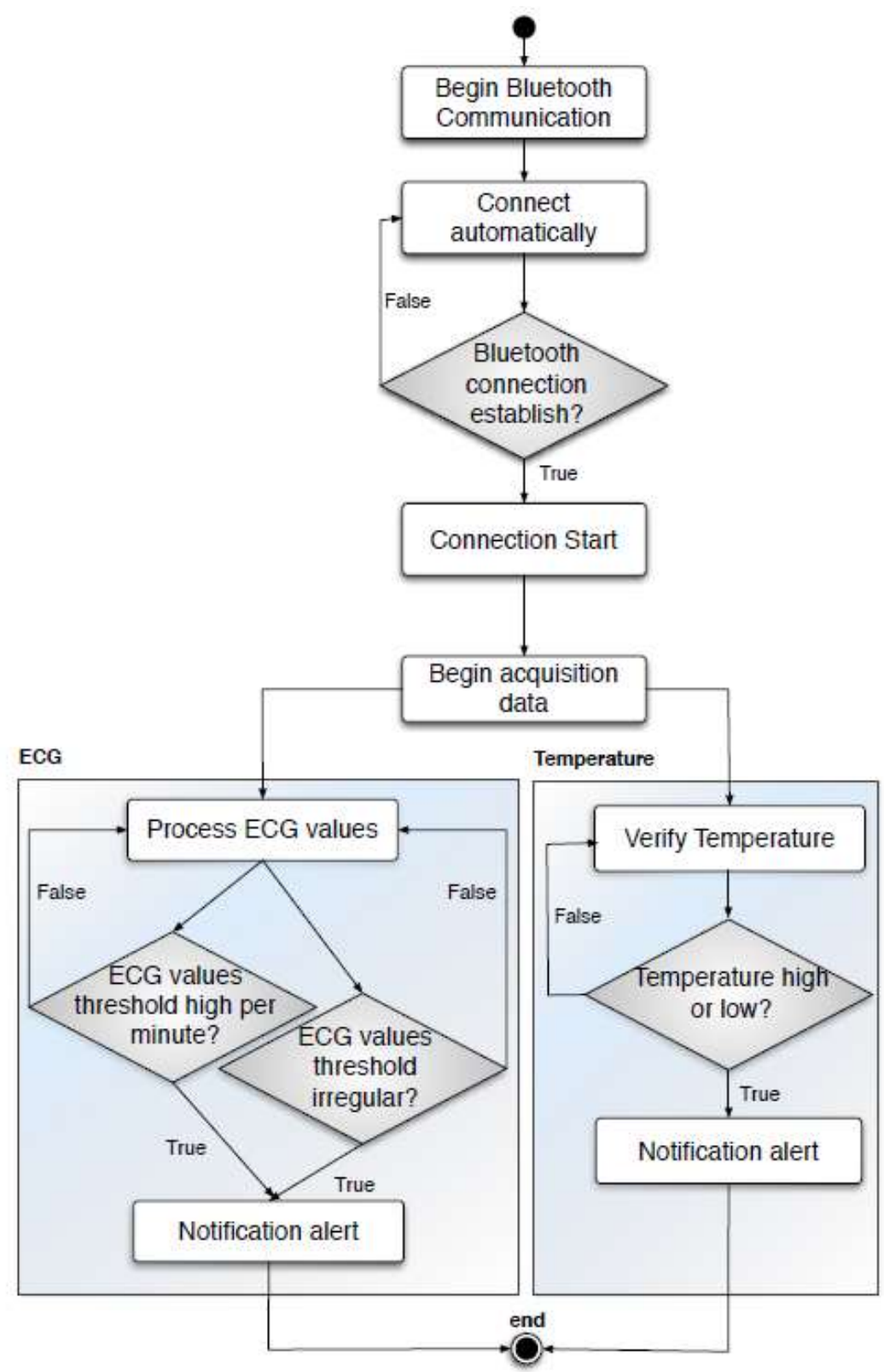

Figure 9 - Activity diagram illustrating the ECG and Temperature prevention, and detection procedures. 
Therefore, the first step consists in the establishment of a Bluetooth connection. Once the connection is achieved, data collecting begins for ECG and temperature values. The collected data is analyzed and if it has expressive changes (for example, ECG values threshold per minute higher or irregular values), an alert is sent. In the same way the collected temperature is analyzed and if the values are very high or low, an alert is also sent to the elderly and/or to the caregiver.

\subsubsection{Falls Detection}

The fall detection process uses the accelerometer sensor data and using multi-threading to the simultaneous combination of several tasks and several sensors readings.

In Figure 10, it is presented the activity diagram of falls detection system, which represents the data flow and the sequence of the application. 


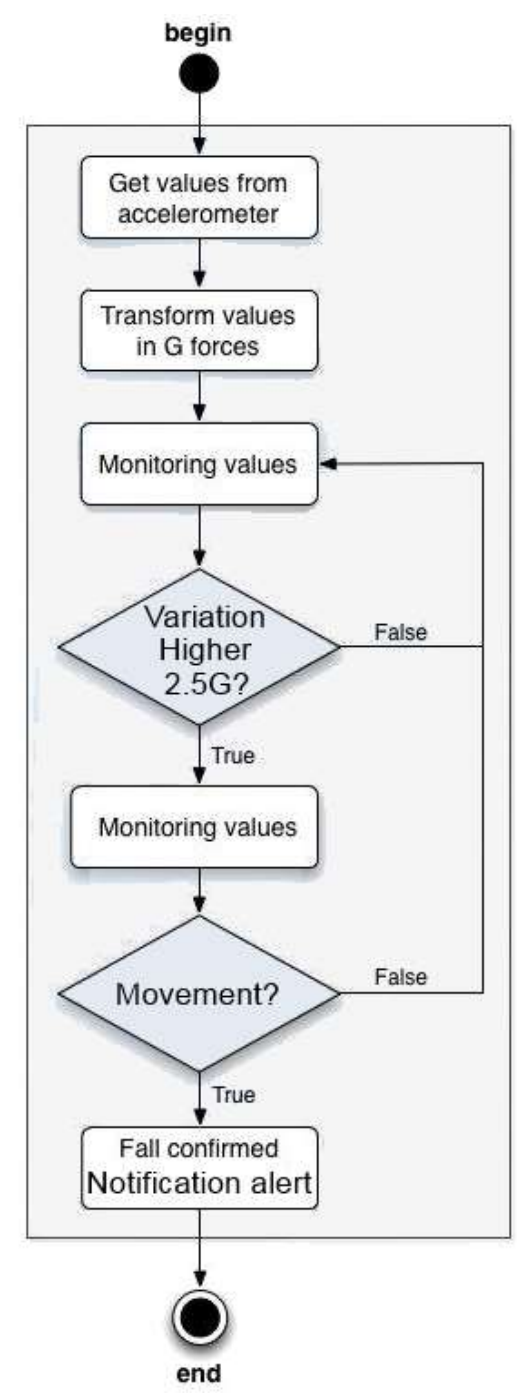

Figure 10 - Falls Detection activity diagram.

Figure 10 represents the workflow as the beginning process start of collection of the acceleration values from the accelerometer and these values of axes $X, Y$, and $Z$ received from accelerometer was transforms in $G$ force, using the equation 1 . The $\mathrm{G}$-force is a measure of acceleration of a freely falling body.

This task will always be active and thereby can monitor the user activity. If $\mathrm{G}$ force is superior to $2.5 \mathrm{G}$, after an interval of 10 seconds some movement occurs, it is declared as false alarm. In case it is no activity, was possible the user may be unconscious or immobilized and in this case they need help. At that moment the device launched a notification it is shown on main screen. 


$$
G=\frac{\sqrt{x^{2} \times y^{2} \times z^{2}}}{9,8}
$$

Equation 1. G force calculation.

\subsubsection{Processing and Information Visualization from Sensors}

In this proposal as above mentioned, there are several sensors that can be individually activated, such as, BVP, EMG, and GRS bio-signals measurement but also some inertial sensors as gyroscope and magnetometer. This remaining sensor allows visualization in a simultaneously way in Figure 11. 


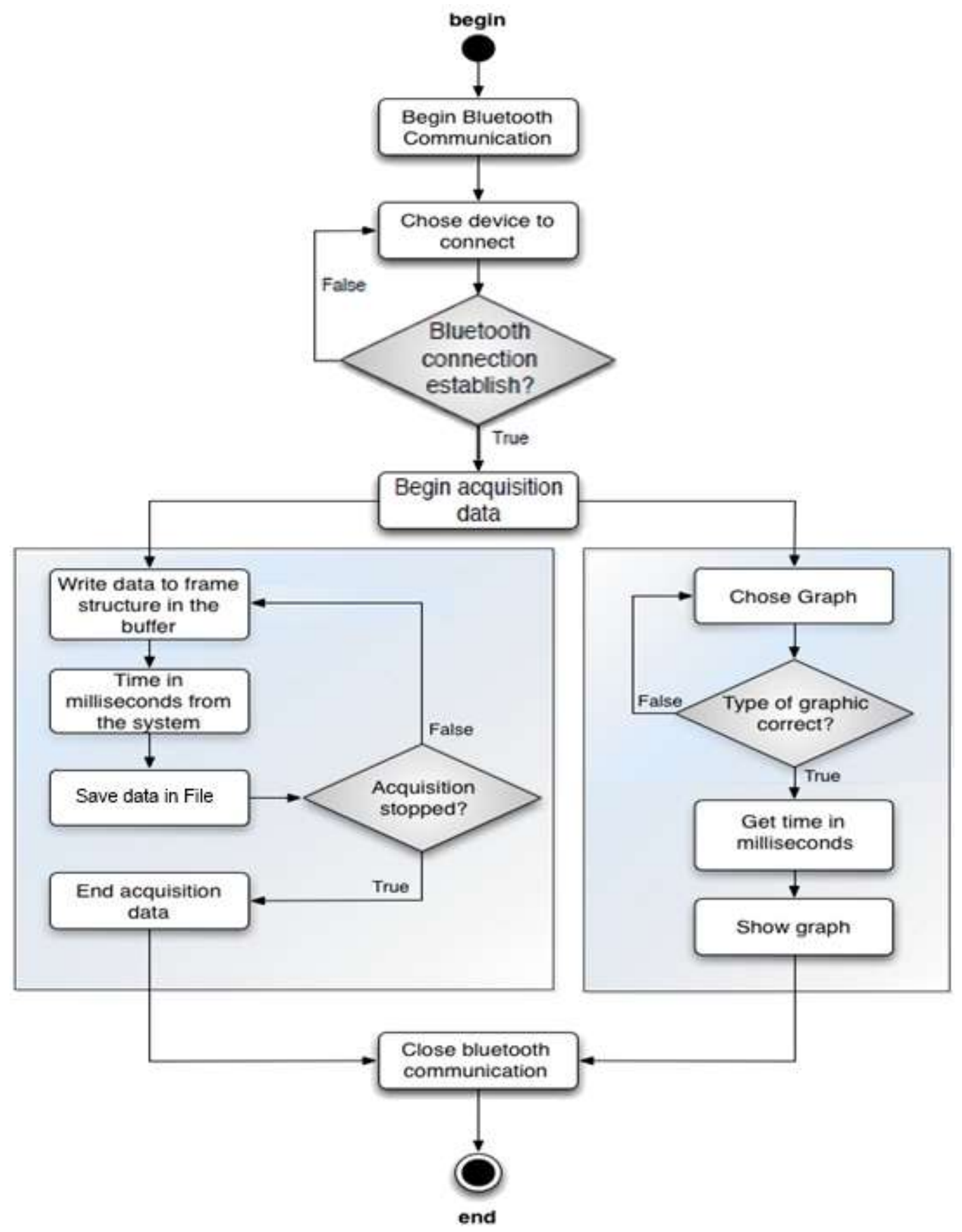

Figure 11 - Bluetooth Connection, Receive, Store and processing data, other Show Charts activity diagram.

These sensors can also be presented in a smartphone allowing the detection of significant changes in user motion. Therefore it enables fall detection through the accelerometer. The proposal has the ability to use the smartphone or tablet GPS to provide the real-time location of the user. 
After communication Bluetooth starts the system and creates threads for each sensor, one for the data acquisition and another for the visualization of the values in a graph. When a connection is established, acquiring data will start from the connected sensors. The received data is saved on a file, for each sensor. It is also possible to see the graphs according to the values from the sensor, individually.

\subsection{Structural Diagrams}

Structural diagrams represent the oriented object elements of a system and the most used structural diagram is the class diagram.

Class diagrams show a collection of several elements such as classes, their types, and their relationships. The various elements are represented statically and it are used as a template for the creation of objects. 


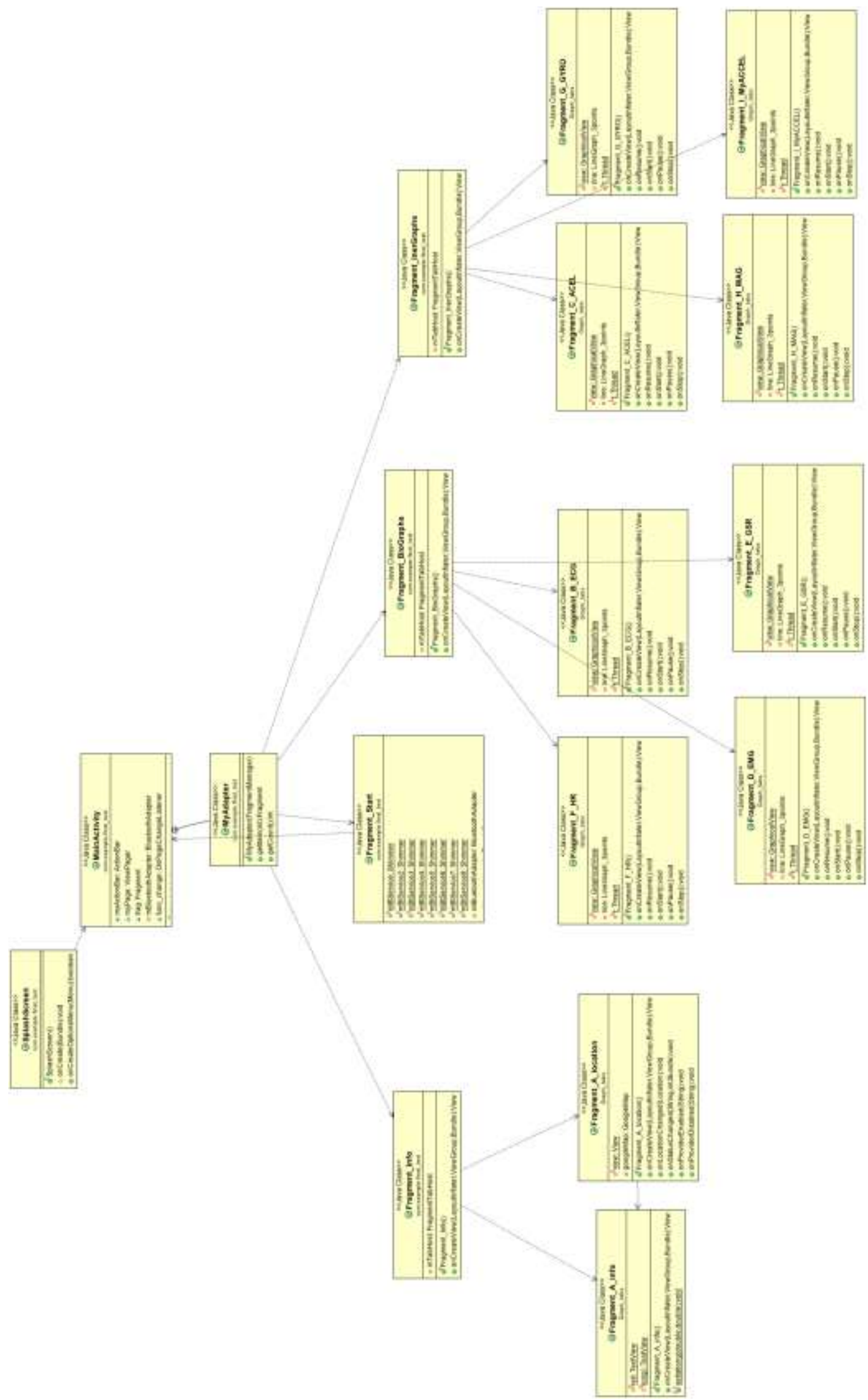

Figure 12 - Structural diagram with Bluetooth connection for health sensors, Falls Detection, GPS location and to display health data charts. 
Figure 12 presents a class diagram with proper attributes and methods developed for the Android System and an hierarchical representation of the classes' dependencies of a main class called MainActivity.

Note: Structural diagram (Figure 12) attached at the end with a picture oversized.

\subsection{Used Technologies}

The two AAL solutions presented in this dissertation were developed in the Java programming language.

The first application the IDE chose to develop was NetBeans which aimed the functionality of a watch and a chest strap. This language allowed developed applications WORA (write once, run anywhere), not to be necessary to compile the application for different operating systems. This is due to the fact that JAVA language compiles its applications to byte code instead of the native code of the operating system, allowing any computer with Java Virtual Machine installed. JAVA is a widely used language so it has some advantages. Its starting technology is free and it is possible include new APIs and free IDE's. Object-oriented programming uses allow it to run on multiple platforms without the need for code changes. The disadvantages we can mention are that the compilation is a bit slow in comparison to other languages and JAVA applications are a little slower to load. 


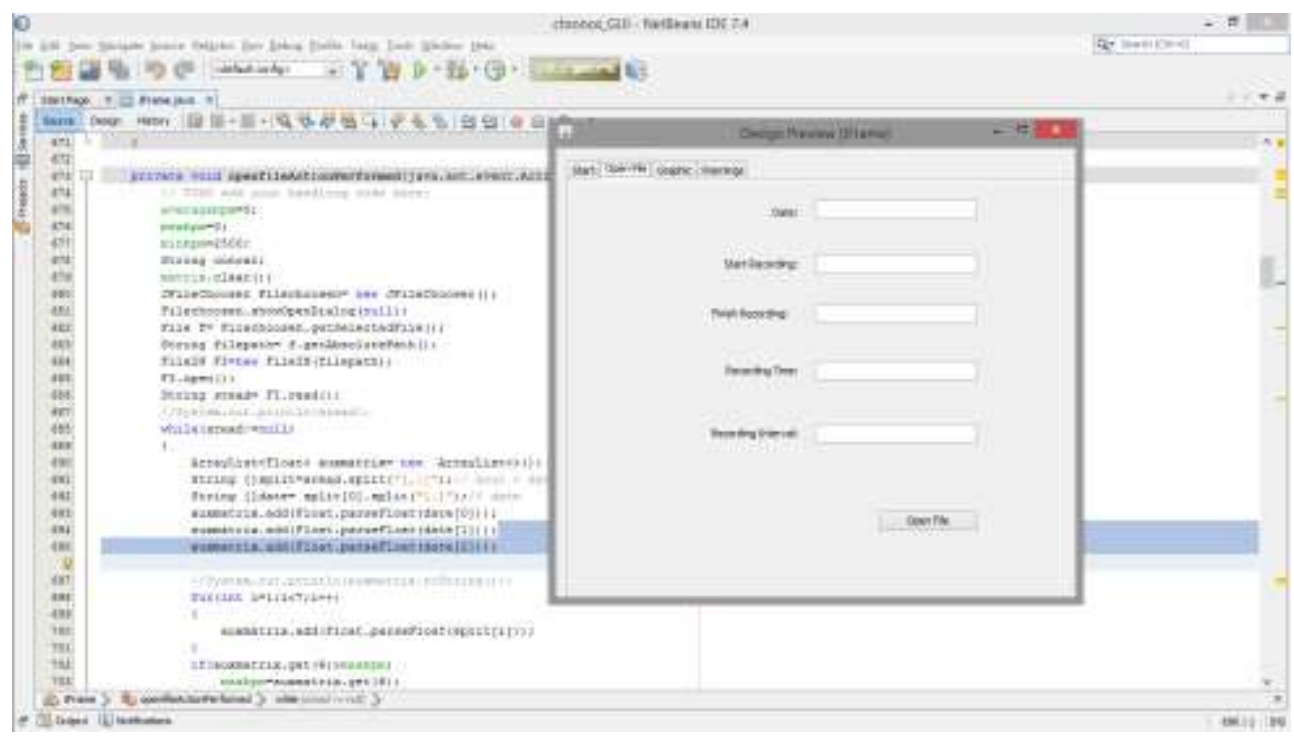

Figure 13 - Development of the application on IDE NetBeans.

The knowledge of Android architecture is essential in order to be possible to design and develop robust and consistent solutions towards Android OS. The following Figure 14 shows the main system components of the Android OS.

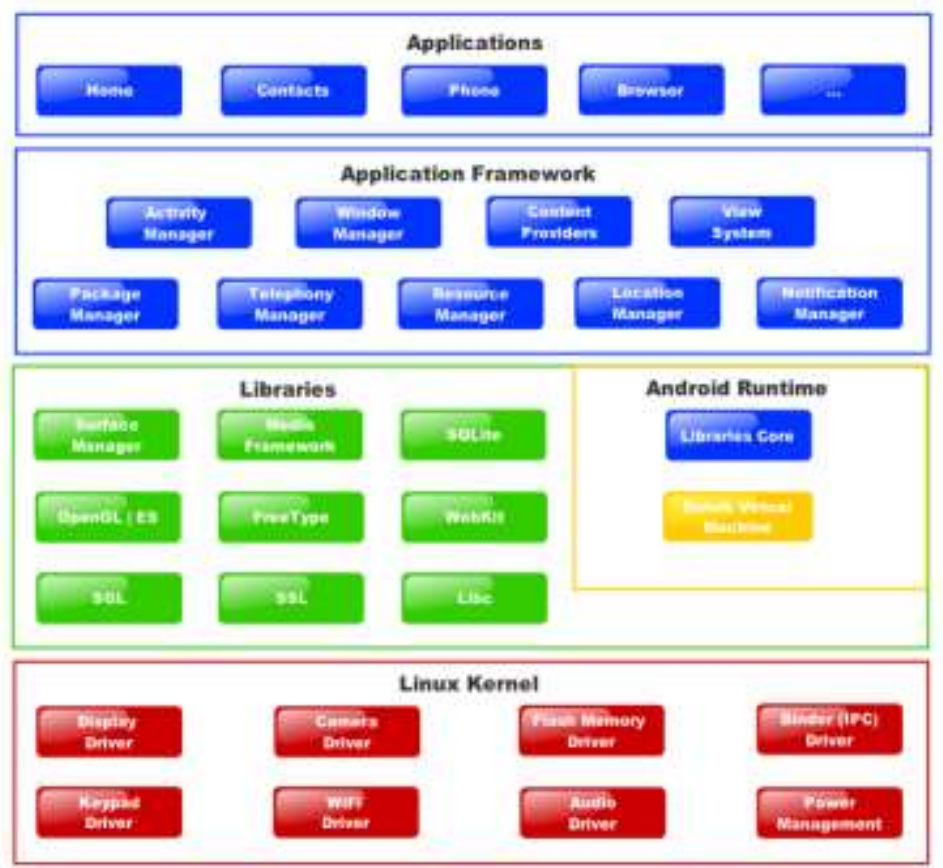

Figure 14 - Illustration of the Android OS architecture. 
Activity lifetime in android takes activity management in a stack that accomplishes the different activities that the application has. Those activities have four main states (active, paused, stopped, and dropped). When a new activity is created, it is placed on the top of the stack and becomes the running activity. The previous activity remains below, in the stack, but this mobile application may have different activities need simultaneously and each activity can contain Threads to perform multiple tasks. For example, if map activity is launched in front of Falls Detection activity this method does it work in the background. To exit the application you just have to destroy the activities running in the application.

The second application given aims mobile devices running Google Android operating system, aiming to be a flexible platform, and an open source that will work different types of smartphones. To make apply mechanisms possible by developing the system, Android Software Development Kit (SDK) [91] is used. This instrument offers the necessary tools to develop android applications to run in smartphones and tables. In this case the programming language used is JAVA. The IDE chosen for development is the Eclipse in combination with the Android development tools (ADT) plugin, designed to extend Eclipse capabilities, providing debugging options or even test emulators, and has the largest community of Developers and a large set of detailed documentation of all features. 


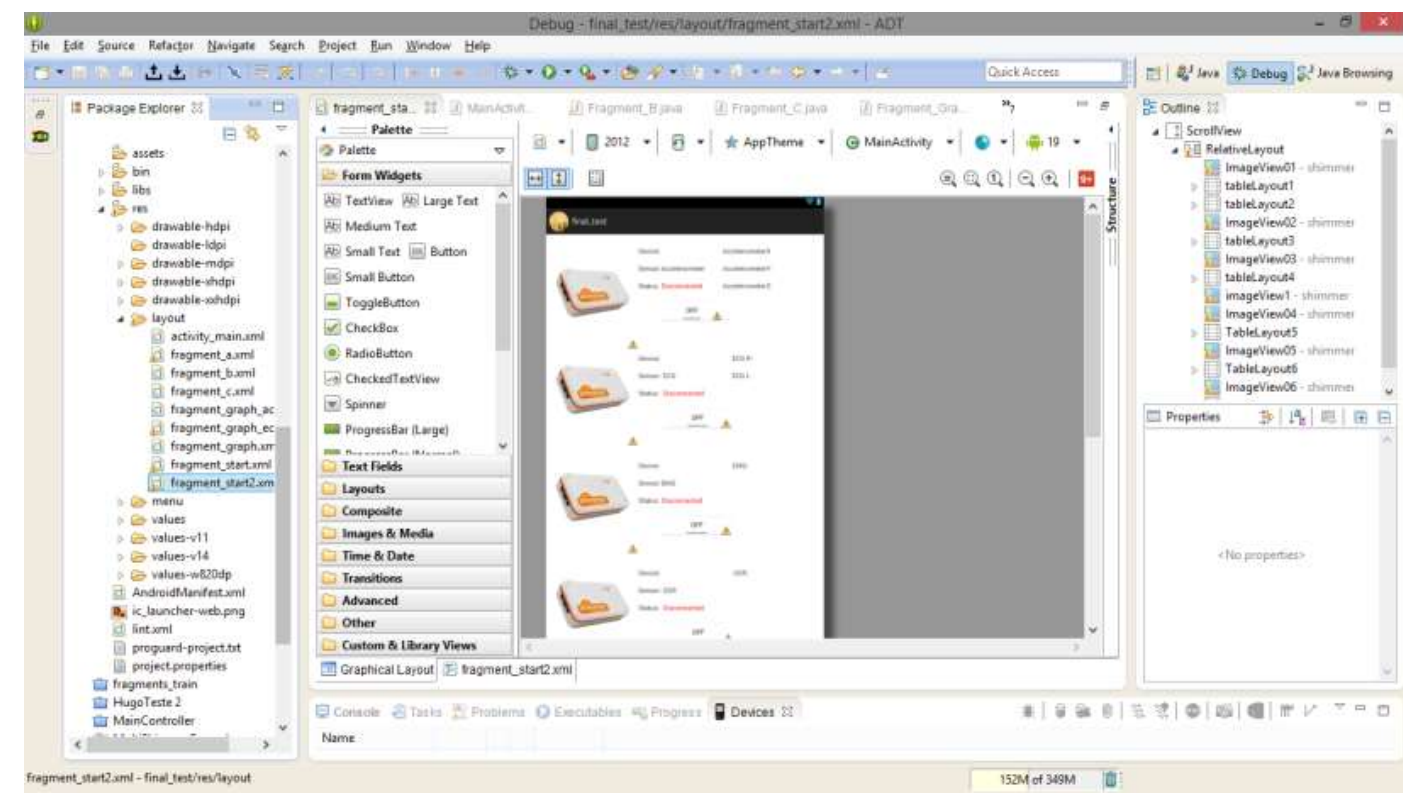

Figure 15 - Development Environment ADT.

Typically, all Android devices offer networks connection through Wi$\mathrm{Fi}$ and Bluetooth. In this case there is an important requirement for the proper functioning of the application and it receives data directly from the tablet "Nexus 7" accelerometer and all the necessary equipment to fix the seven sensors Shimmers2r, through Bluetooth.

\subsection{Summary}

In this chapter the requirement analysis was focused in all system behaviors and essential features. Thus, Section 3.1 presented the system essential modelling requirements, Sections 3.2 and 3.3 presented behavioural and structural diagrams to design mobile solution. Section 3.4 discussed used technologies. Finally, this Section summarizes the chapter. 


\section{Devices used on the Prototype}

The goal of this chapter was the experimental systems of devices used in the prototype and the proposed mobile health sensors solutions fit to do research. Both are developed and applied to the AAL mobile health solution, like Biofeedback monitoring, monitoring movements, falls' detection, and in a general way are prevention mechanisms to help older people and this concerning the highest specifications to the AAL mobile environment. Those will be shortly introduced and technically described by the projected health mobile solutions, in Section 4.1 the Texas eZ430Chronos system and in Section 4.2 the Shimmer Research solution. Both are capable solutions for biofeedback monitoring and have a 3-axis accelerometer and some physiological sensors to collet vital data from the patient's body.

To test the devices showed below it is necessary to refer the Android mobile devices where the application was deployed and tested. Today, the operating system Android is present in 750 million mobile devices, mainly in smartphones that have become so universal and so powerful and are ready to exceed desktop usage. Those numbers tend to rise for the huge success these devices connecting wirelessly have. Android is the most successful platform [92].

This success is owed also to the fact that these devices have Bluetooth and Wi-Fi connection to support and improve the system on a 
mobile device and also have a low cost and an extensive variety and versatility. There are various versions of Android system but the one that interests us is the Android 4.4 KitKat announced on September 3, of 2013. This application was developed for Android 4.2.2 Jelly Bean and was deployed and tested on tablet Nexus 7, but also experimented on other previous Android versions like tablet Samsung Galaxy Tab (Android 2.3.3 version) and other mobile devices, smartphones or tablets available in the market.

\subsection{Shimmer Research Platinum}

The Shimmer Research Platinum solution system, also known as Shimmer $2 r$ Development Kit offers two possibilities to communicate, the standard IEEE 802.15.4 or Bluetooth that allows to send data to mobile devices, smartphones or tablets, without forgetting it flexibility, great portability and autonomy.

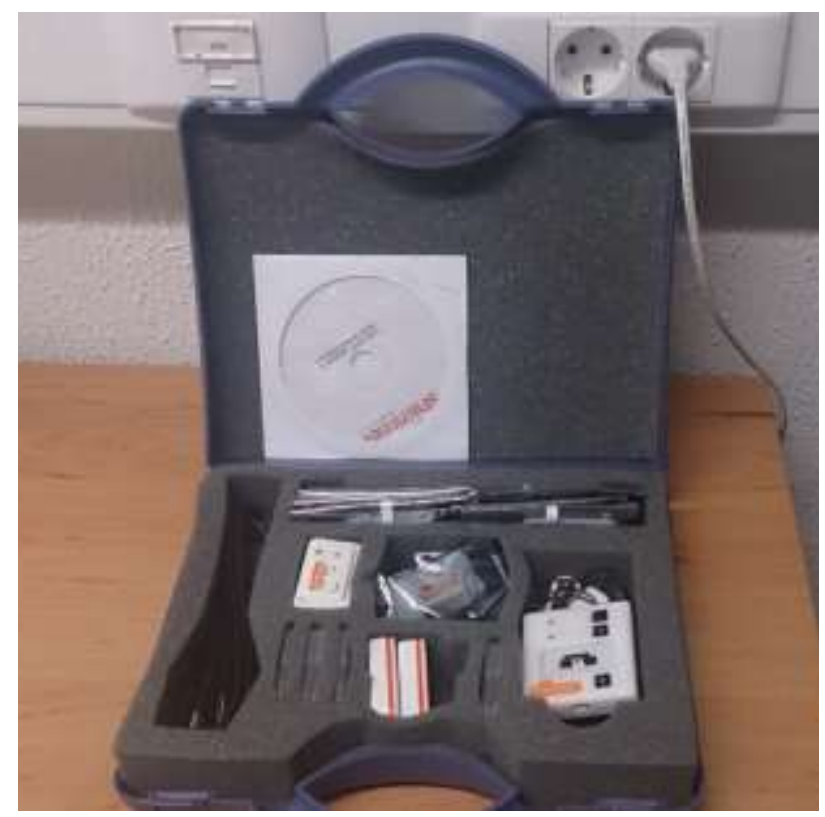

Figure 16 - Shimmer Research Platinum Kit. 
Each basic module sensor is equipped with a low-power microcontroller MSP430 (8MHz, 16Bit) of Texas Instruments, a 3-axis accelerometer for motion sensitive control, the main board also includes a Micro SD slot supporting up to 2 GB of Flash memory for data storage allowing significant amounts of data, a Bluetooth module, a rechargeable 450mAh Li-ion battery along with the advantage of being rechargeable within a few hours and low power consumption. It as also one slot that allows the connection between other sensors. This offers a plethora of body sensor bio-signals measurements, such as the electrocardiogram (ECG), the electromyography (EMG), the Galvanic Skin Response (GSR) sensor, Heart Rate (HR) or beats per minute (BPM), temperature, etc. It is also able to perform respiration demodulation on-chip. It integrates a 10 DoF inertial sensing via accelerometer, gyroscope, magnetometer, and altimeter, each one with selectable range. These modules used TinyOS that a specific mini operating system developed to platforms with restricted memory resources and processing.

The development kit also carries several memory cards, docks for charging and transferring data, straps to attach sensors to patients and also a CD containing complete documentation about each sensor module, the Android API and an installation program that allows us to see all the process of initialization, connection, acquisition and visualization/processing data received through sensors.

\subsubsection{Arrangement of the Sensors in Patients Body}

In the proposal scenario seven types of sensors where used, because it is the maximum number of connections that standard Bluetooth 2.0 allows. As shown in Figure 17. The Shimmer ECG (A) records the electrical impulses through the heart muscle, having as starting point four electrodes set $(C)$ on the chest of the patient. It is also possible to record data on bedridden or home users, or even during exercise to provide information of 
the patient's heart in real time. Furthermore, a temperature sensor (B) allows gathering information of a patient's temperature when the sensor is placed in close proximity to the body. The digital interface signal experiments several sensors for validation purposes.
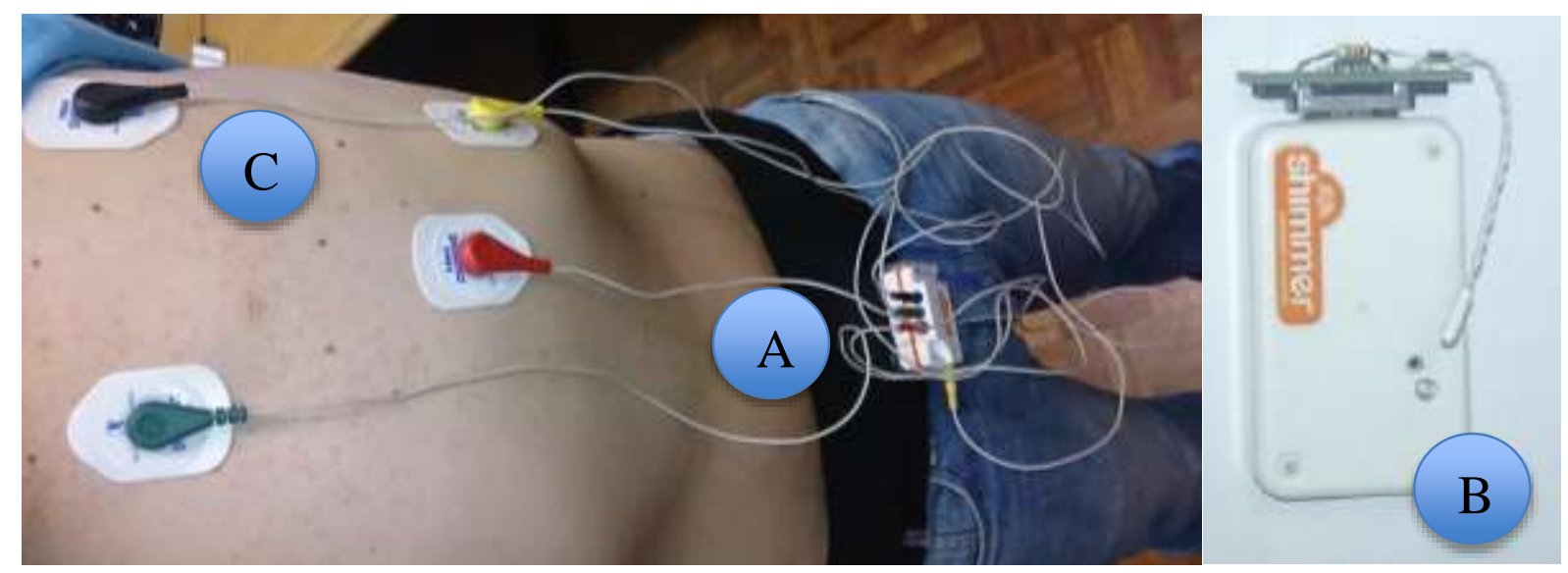

Figure 17 - Illustration of the body sensors - Shimmer: A) Electro-cardiogram; B) Temperature; C) Electrodes for Electro-cardiogram.

In the next Figure 18 it is possible to see all the hardware of the system together, all the seven sensors Shimmers2r and one tablet Asus Nexus 7 assigned by NetGNA group. All sensors are connected to a voluntary and turn on through Bluetooth connection to the tablet and a set of tests and simulations are carried out to obtain several kinds of values and some simulations of falls (Figure 19). This simulation took fifteen minutes. All the connections run ok during the simulation and all data was stored in the device. 


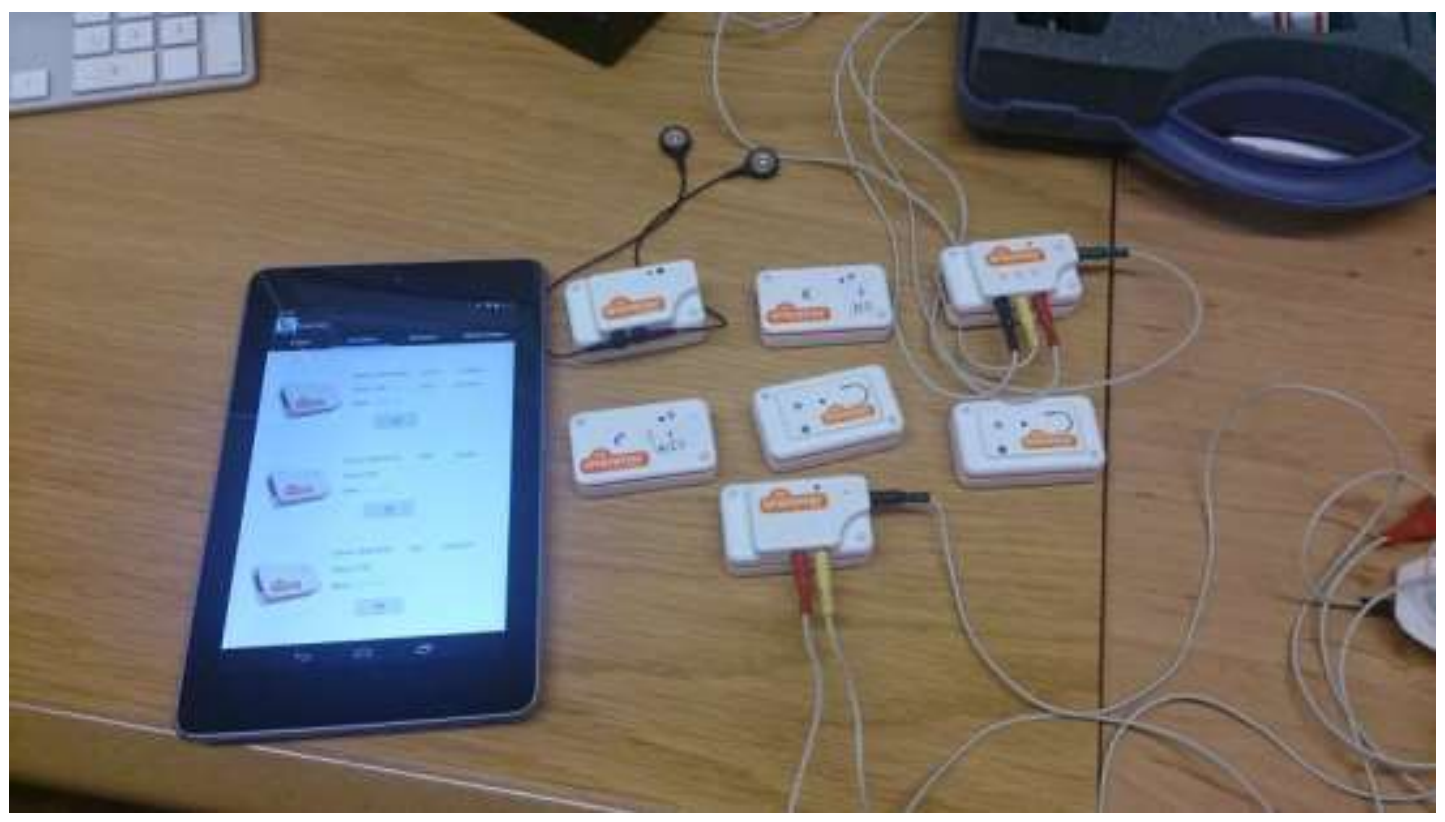

Figure 18 - Material used for the development of the solution.

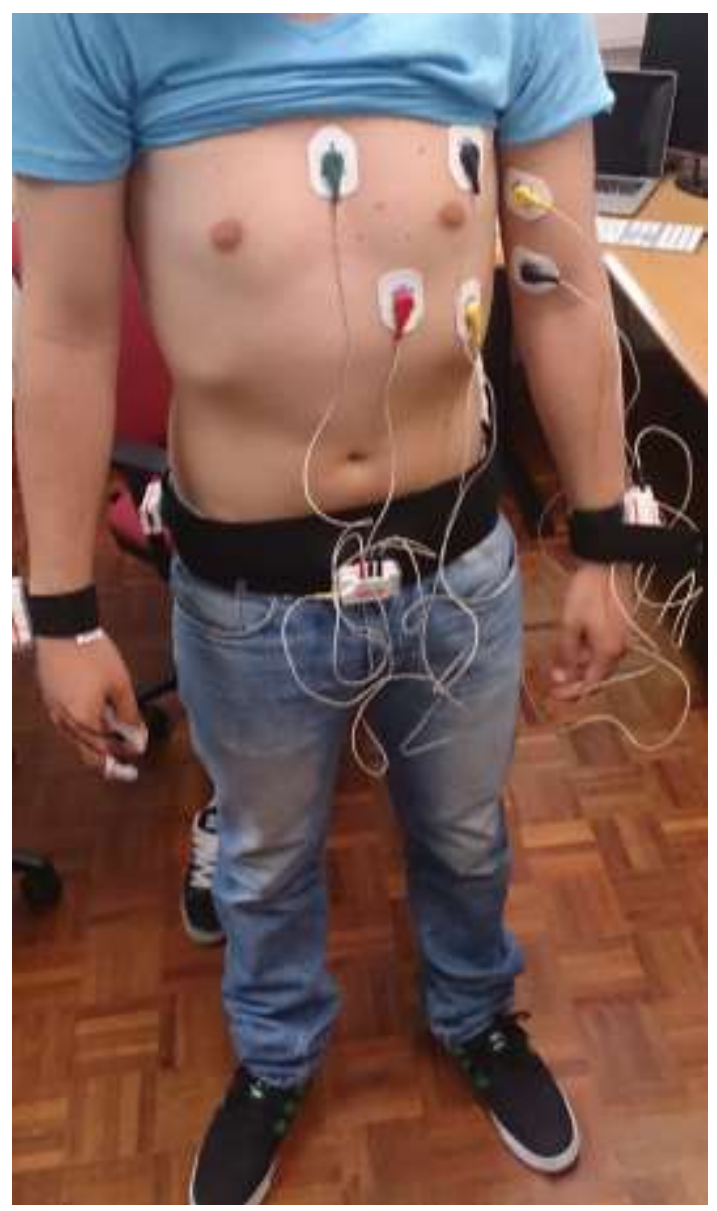

Figure 19 - Connecting Sensors Shimmer in last test. 
The Shimmer Kit is a good development tool because is adapted for wearable solutions and has lots of documentation about the Android API [93] in a way that reduces the time development in applications where sensors can be applied to a wide range of scenarios, such as, motion detection, heart function monitoring, premature ventricular contraction, atrial fibrillation, abnormal rhythm detection, fatigue analysis, muscle activity, sport techniques, gait and posture disturbance, tremor analysis, and orthopedics biomechanics. All these solutions can be controlled in real time monitoring or over data acquisition extended periods of time.

\subsection{Texas eZ430-Chronos}

The Texas eZ430-Chronos research system offers flexibility and adaptively, because it stands out for being the first reprogrammable watch and it also allows wireless communication with a Chest Strap, USB Access Point RF (Radio Frequency) or with a pedometer. These accessories have a big communication range with the watch and still are portable, autonomous and easy to use.

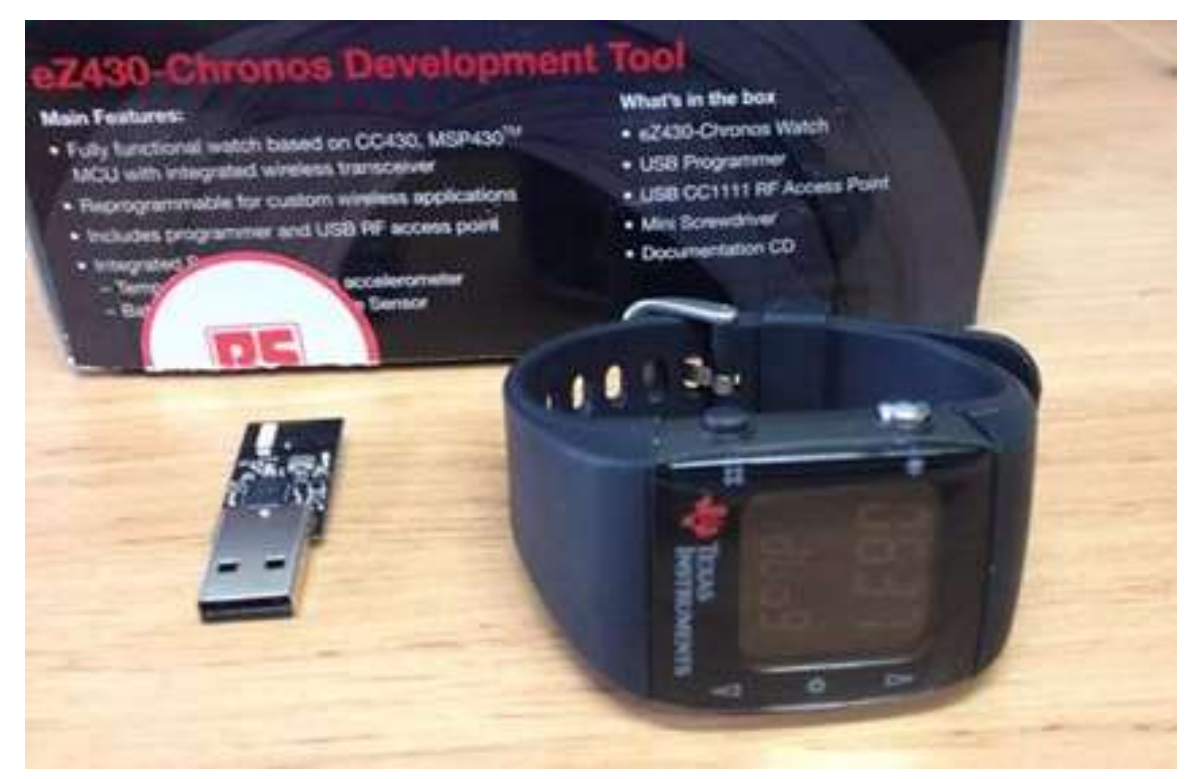

Figure 20 - Texas eZ430-Chronos Development Kit. 
The eZ430-Chronos watch, presented in Figure 20, was the first wearable development kit released worldwide and needs disassembled to be reprogrammed with a custom application taking into account the needs of given users. It has been required updating the firmware, because otherwise it would not be possible to access the recording mode data (LOG). This feature LOG allows a maximum of 2557 entries $(8 \mathrm{~kb}$ of information), taking into account that BPM values, temperature and time are saved.

The watch includes a 96-segment LCD display, incorporates a lowpower microcontroller, the CC430F6137, an integrated pressure sensor, a temperature sensor, a heart rate monitor through a chest strap, and a 3axis accelerometer for motion sensitive control ability to operate on a central hub for nearby wireless sensors (such as pedometers). The development kit also carries a USB Access Point to reprogram the watch and a CD containing documentation and two IDE's a IAR Kick Start and Code Composer Studio v4

Collected signals from the sensors were stored on the watch, and is possible to transmit this data via USB Access Point RF to computer. All signals can be showed in real time. It was created an application that stores information about the temperature and heart rate from the patient's body using the chest strap.

These instruments usually have a set of restrictions but, in this case, the watch performed well because it did not present any problems of flexibility, ergonomics, or discomfort resulting from time extended handling and use. Considering this, it is important to underline that the watch and chest strap autonomy are particularly large. During the whole process of acquiring data there was no need to change any batteries of either the watch or chest strap. For the CR2032 Coin Cell Lithium Battery, referred on the manual and according to its analysis, it was possible to observe that for a continuous communication in BlueRobin Rx Mode (communication: chest strap to watch) the battery lasts approximately 6.2 months, but if only needs 1 hour of daily use, so, the battery can work for 2 
years. Otherwise, the SimpliciTI SYNC mode (communication: watch to computer) is more expensive energetically because of its continuous communication. The battery takes about 8 days to empty but 1 hour per day use takes approximately 5.4 months. The SimpliciTI SYNC mode is much more energy eater, this is not a big problem because it is only used to send data to a terminal and this communication is done in seconds. Furthermore noted it is possible to control some functionalities through the computer and manage settings as data is conducted. However, it has some disadvantages. It could have bigger memory capacity and capable of simultaneous communications.

\subsubsection{Arrangement of the Sensors in Patients Body}

Placing the sensors correctly in the patient's body is essential to have accurate readings. In Figure 21 the sensors are placed in a specific area of the human body. The chest strap was placed on the chest (heart area), and on the wrist we should put the watch, thereafter accessing the mode "LOG" on the watch and start recording data. When completed the recording phase, data is transferred to a computer for subsequent analysis by one specific application.

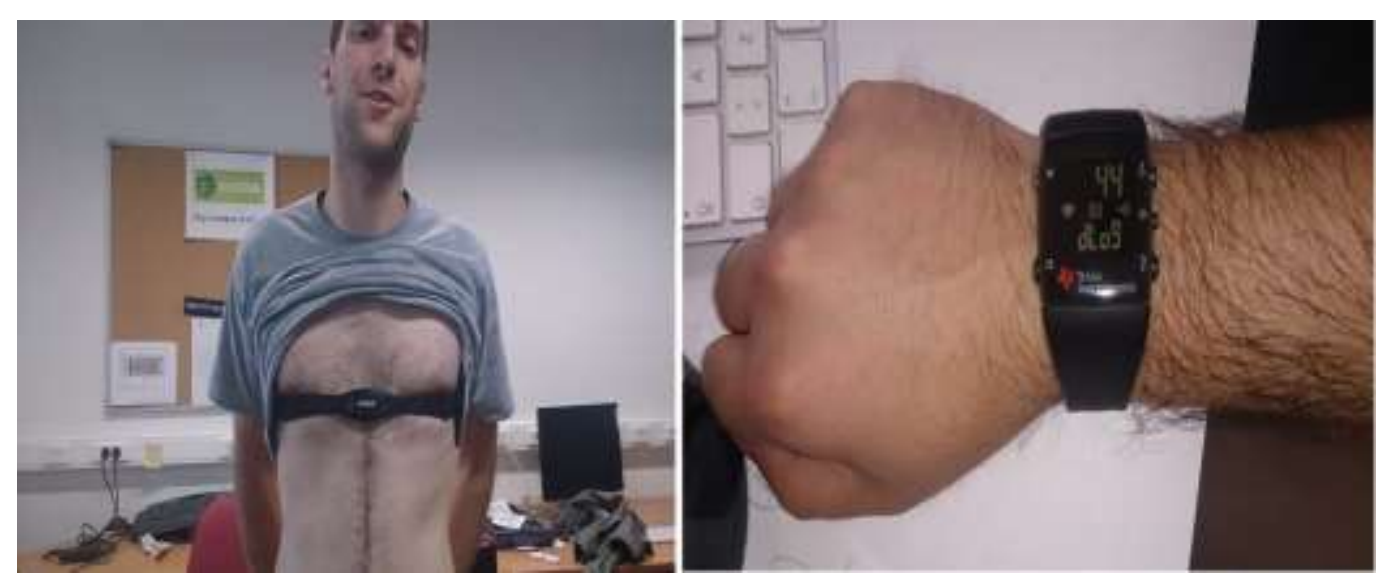

Figure 21 - Placement of the Sensors in the Human Body. 
Sensor reading was able, in our case analyses to read the temperature and BPM, in a defined time range between samples and it was performed in ideal conditions of data acquisition. But these sensors are flexible, robust and allow us to take samples under extreme conditions.

The communication between the watch and the computer has a good range, approximately 100 meters (tested in open field with line of sight), but indoors setting with RF activity it is able to achieve a range of 10 meters without equipment causing interference.

\subsection{Summary}

In this chapter, it was presented the most important technical characteristics and the functionalities of the used devices that performed the AAL mobile solution prototype. Texas eZ430-Chronos research system was presented in Section 4.1. In Section 4.2 another health sensors kit (the Shimmer Research Platinum Kit) was showed. However, this mobile solution can improve to other sensor systems. 



\section{AAL Mobile Solution}

\section{Demonstration}

This chapter will be focused on the flexibility of mobile devices that enable more reliable and customizable mobile tools. Bio-signals monitoring allows the identification of possible and meaningful temperature, heart rate, or BVP changes. Additionally, the system can identify heart diseases like arrhythmia as well as other cardiac alterations. Moreover, the caregiver is able to define the risk degree of the patient under monitoring and defining three levels of surveillance: low, normal, and critical [94] [95].

The system Architecture introduced and described in Section 5.1 presents all system modules for biofeedback monitoring and falls detection. Then, in Section 5.2, it is showed the graphical interface adapted for elderly people. Section 5.3 performs and explains the biofeedback monitoring tools. Section 5.4 focuses on the falls detection with warning alerts in dangerous health circumstances.

\subsection{System Architecture}

This section describes the system architecture considered for AAL environments with mobility support. Figure 22 illustrates a scenario with 
main actions set for the communications process with the mobile application and the healthcare system, in real time.

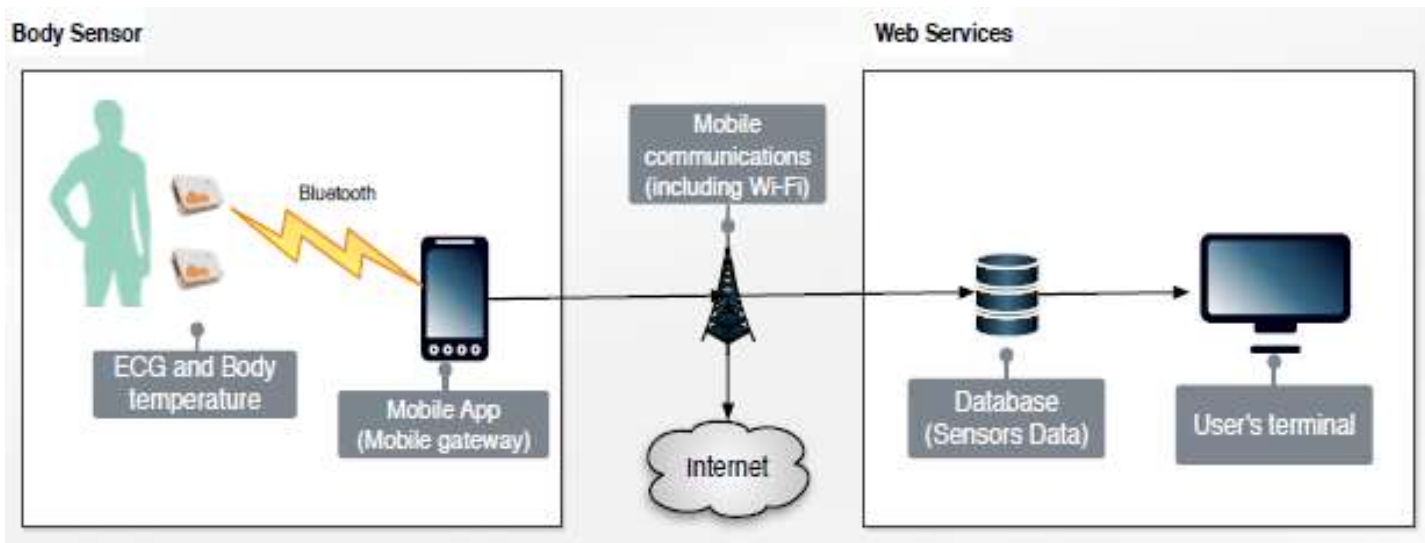

Figure 22 - Illustration of the system architecture, using ECG and temperature body sensors and external access through Web services for data visualization and storage.

The system architecture of the mobile App allows data collection from sensors and external access from user's terminal through an Internet connection. It receives all data from the sensors placed beside the user body in order to obtain the vital parameters and extra information that is sent through Bluetooth to a smartphone or tablet. The collected data is analyzed and sent to a remote database. It can be stored for a more extensive analysis of the medication in order to improve or even adjust it. The body sensors and Web services modules facilitate the information sharing through a simple interface, which is properly identified by users with specified permissions.

The fall detection module was presented in the last phase and it is responsible for detecting some movements that have dangerous accelerations in order to detect user falls and also to provide user location in alerts with data GPS navigation system and view on a map.

Therefore, the information sharing is safely and effectively performed. Measurements can be tuned according to different levels that the caregiver defines most relevant. Moreover, this is a flexible solution, fits in a real context and allows the integration of new sensors, if needed. 


\subsubsection{System Architecture - Watch}

Figure 23 presents the same approach but in offline mode. After a starting configuration in which the type of data that we want to store and the time interval between samples is indicated and it stores data information like heart beat and temperature to an offline database for later analysis. Offline bio-signal data is collected through radio frequency. Afterwards, a health care professional can analyze the stored data in order to verify whether the patient eventually needs any medication change or even another type of intervention.

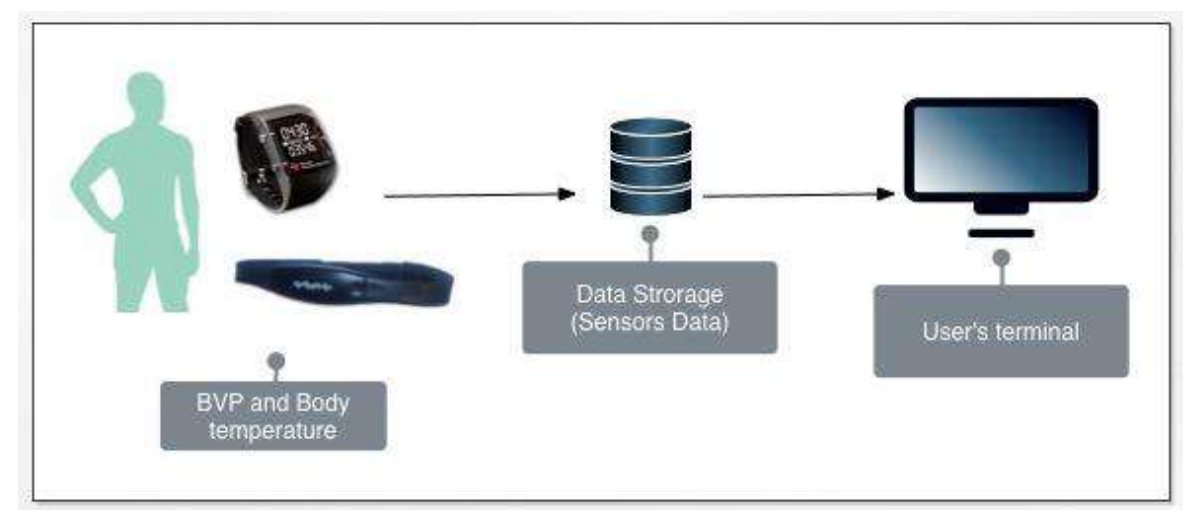

Figure 23 - Illustration of the offline system architecture considering the capture of blood volume pulse (BVP) and body temperature values.

\subsection{Graphical Interface}

This section focuses on demonstrating the solution in a real scenario and these solutions were developed thinking about elderly people showing one user's interface friendly.

The application uses easy structure, large menus and some natural buttons. All the important physiological data is running on the background and alerting the user when it is necessary. Figure 24 shows the initial screen application window. 


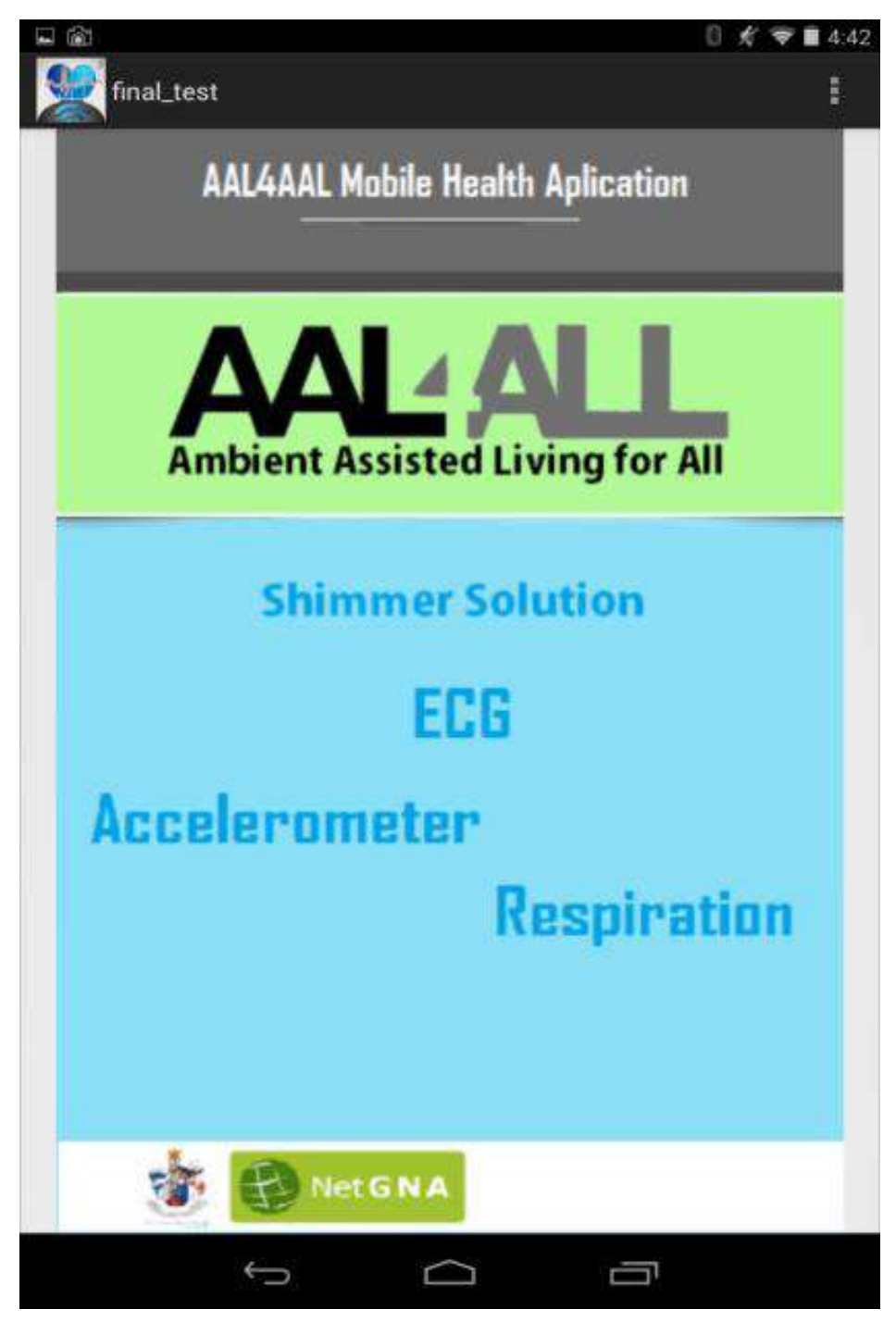

Figure 24 - Initial Screen Presentation.

Figure 25 presents the main page of the application in which the user accesses all personal data information. 


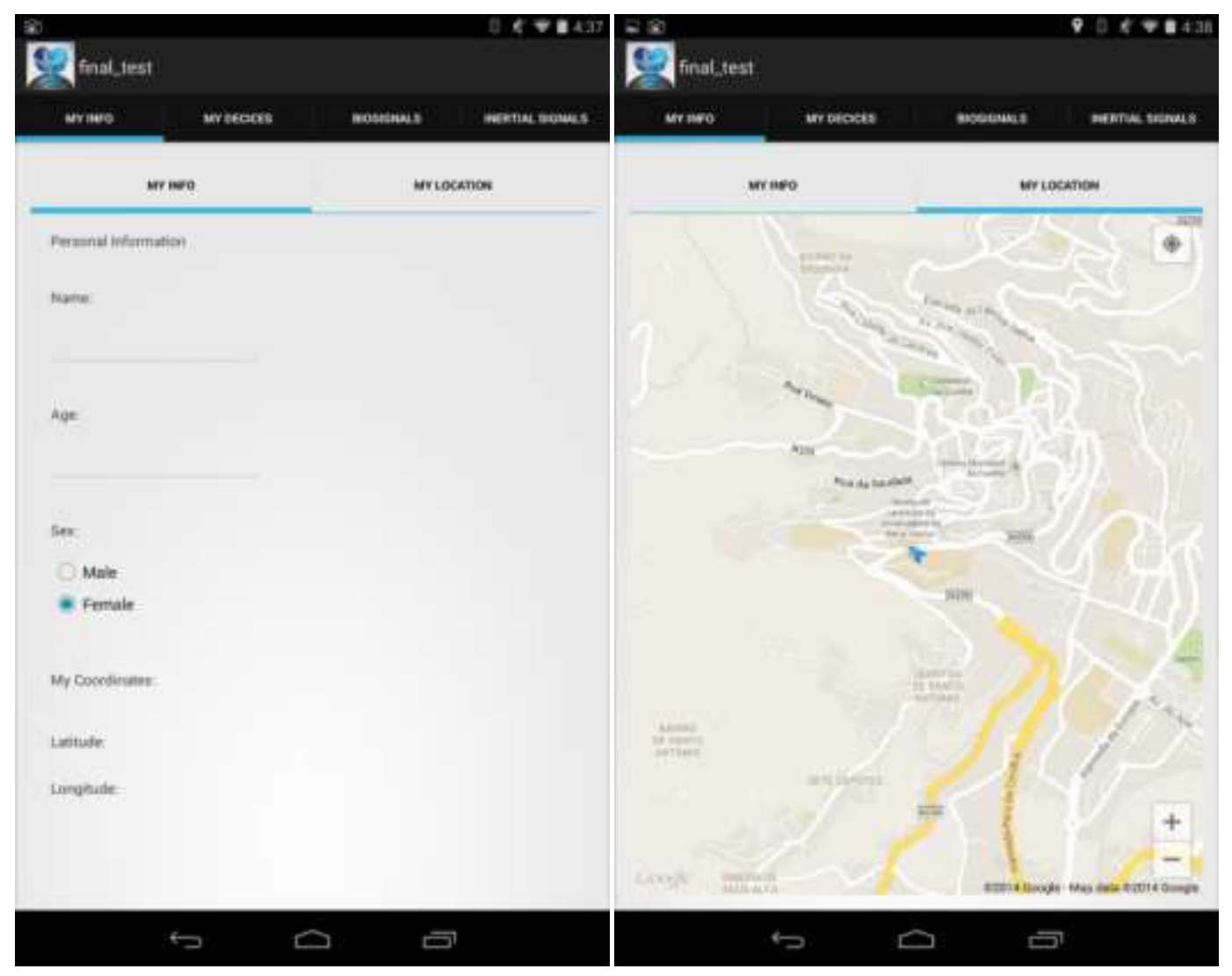

Figure 25 - AAL Mobile Application (personal data information).

After this, the application is ready to initialize the health sensors and it is possible to choose the sensors that will run and be stored. The sensors available are: two tri-axial accelerometer (shimmer and tablet) and it is a device for measuring acceleration, ECG (Electrocardiography) who record change of electrical activity produced by the heart, a EMG (Electromyography), measures the electrical activity generated by muscles, BPM (Beats Per Minute) that counts the beats per minute, one GSR (Galvanic Skin Response) that measures the electrical conductivity of the skin, a Gyroscope which measures the rotation and a Magnetometer that measures distances between magnetic fields. 


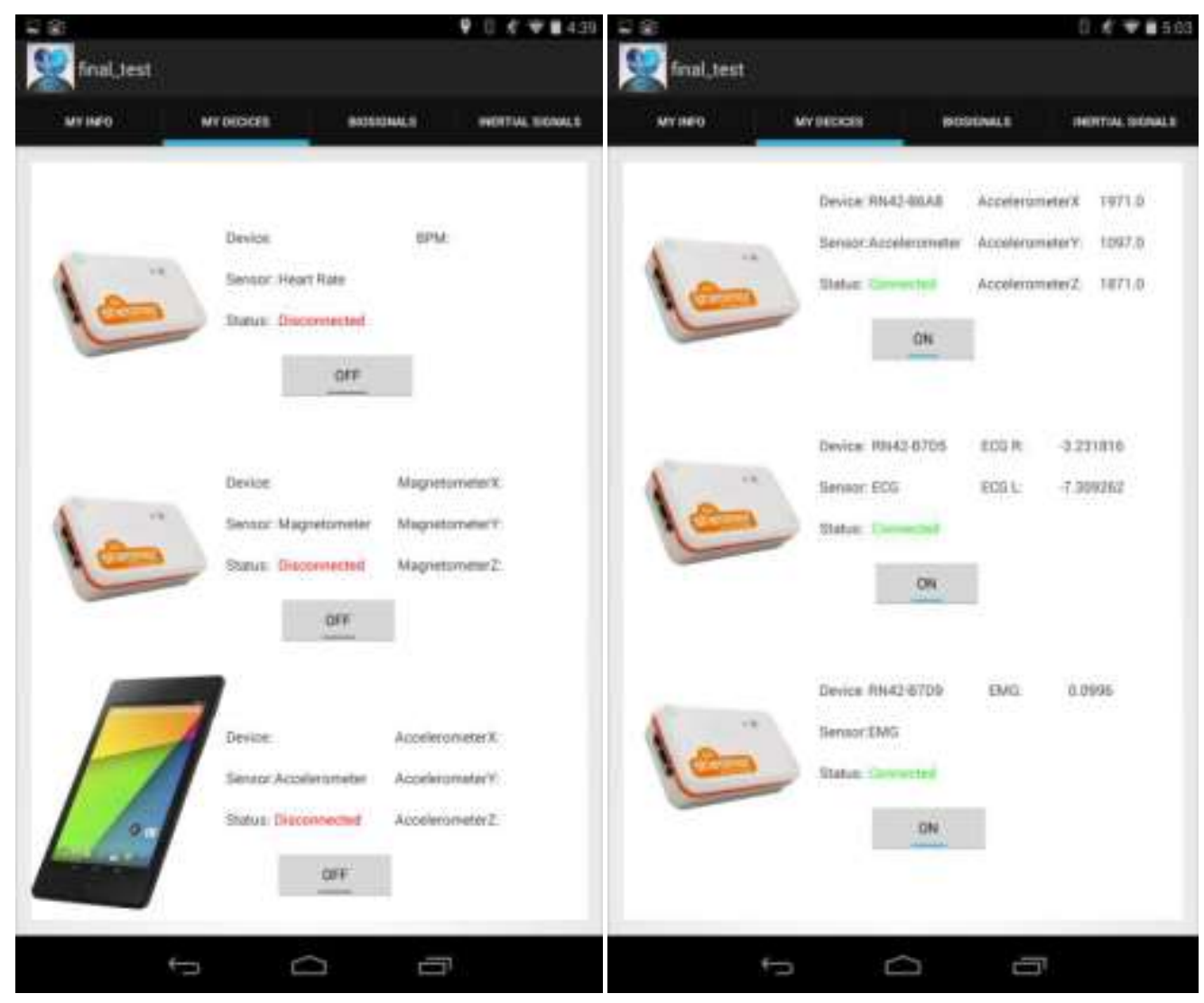

Figure 26 - Fragment with all sensors.

\subsubsection{Graphical Interface - Watch}

This demonstrates the solution in a real scenario and this solution was developed by thinking in elderly people and a user's friendly interface.

The application uses easy structure, large menus and some natural buttons. Figure 27 shows the initial screen application window. 


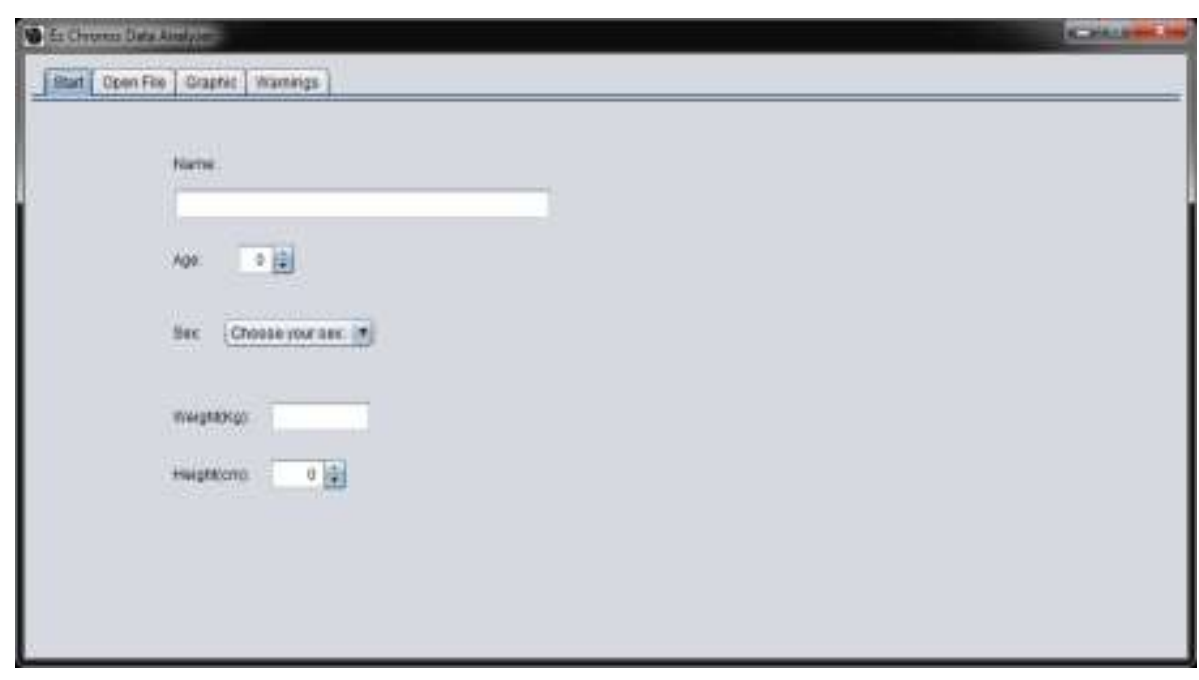

Figure 27 - Initial Screen Presentation (patient information).

The file has the patient information and also two lists with the type of alert, BPM or temperature, the hours are displayed and the value that launches the alert (alerts added later). After opening the file starting and ending dates are displayed, like the total recording time. The file can be opened several times for further analysis.

\subsection{Biofeedback Monitoring Tool}

The monitoring physiological tool is an important component to show both problems and treatments because it is developed to monitor body responses, compile progress data, and reporting them.

The biofeedback status is directly related to certain health issues.

This software was developed to work with two types of sensors the Bio-Signals, like BPM, ECG, EMG and GSR, moreover the Inertial Signals available, like Shimmer and Device accelerometer, Gyroscope and Magnetometer. During a biofeedback session, a patient needs to put some sensors placed next to the skin. Through Bluetooth connection this information is synchronized with a mobile device (smartphone or tablet) for data acquisition. 


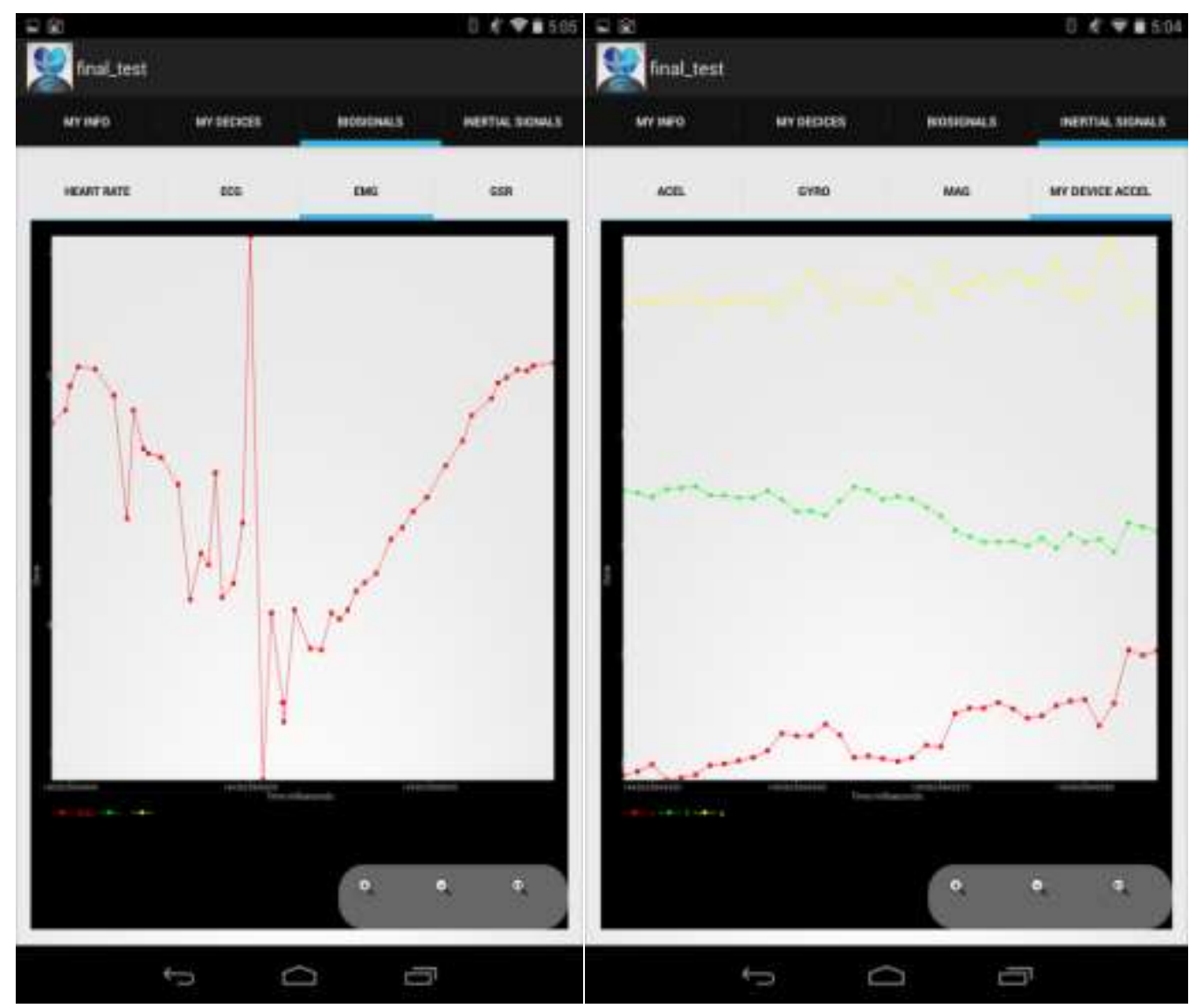

Figure 28 - Fragments of charts.

In Figure 28, a smartphone shows the EMG signal, and My Device ACCEL with values ranging in milliseconds. In a threshold of ten seconds all the graph lines are deleted to be no overlap of information, after the values still printed and it is also possible to do Zoom In and Zoom Out for a particular interval in time. This date timer is obtained from the number of milliseconds that elapsed since midnight, January 1, 1970.

\subsubsection{Biofeedback Monitoring Tool - Watch}

This software was developed to analyse data information about the date recorded by the watch, and it is bound to visualize two types of sensors: the BPM and temperature. It enables the user to configure an alert system and this way visualizes carefully possible risk situations. 
Furthermore it is possible to visualize the initial data information about the patient, the graphs contain BPM and Temperature, but also the data alert saved in the last revision.

By visualizing the next image one might understand that some information graphic has been added on the right, like the maximum and minimum values and average of BPM and temperature.

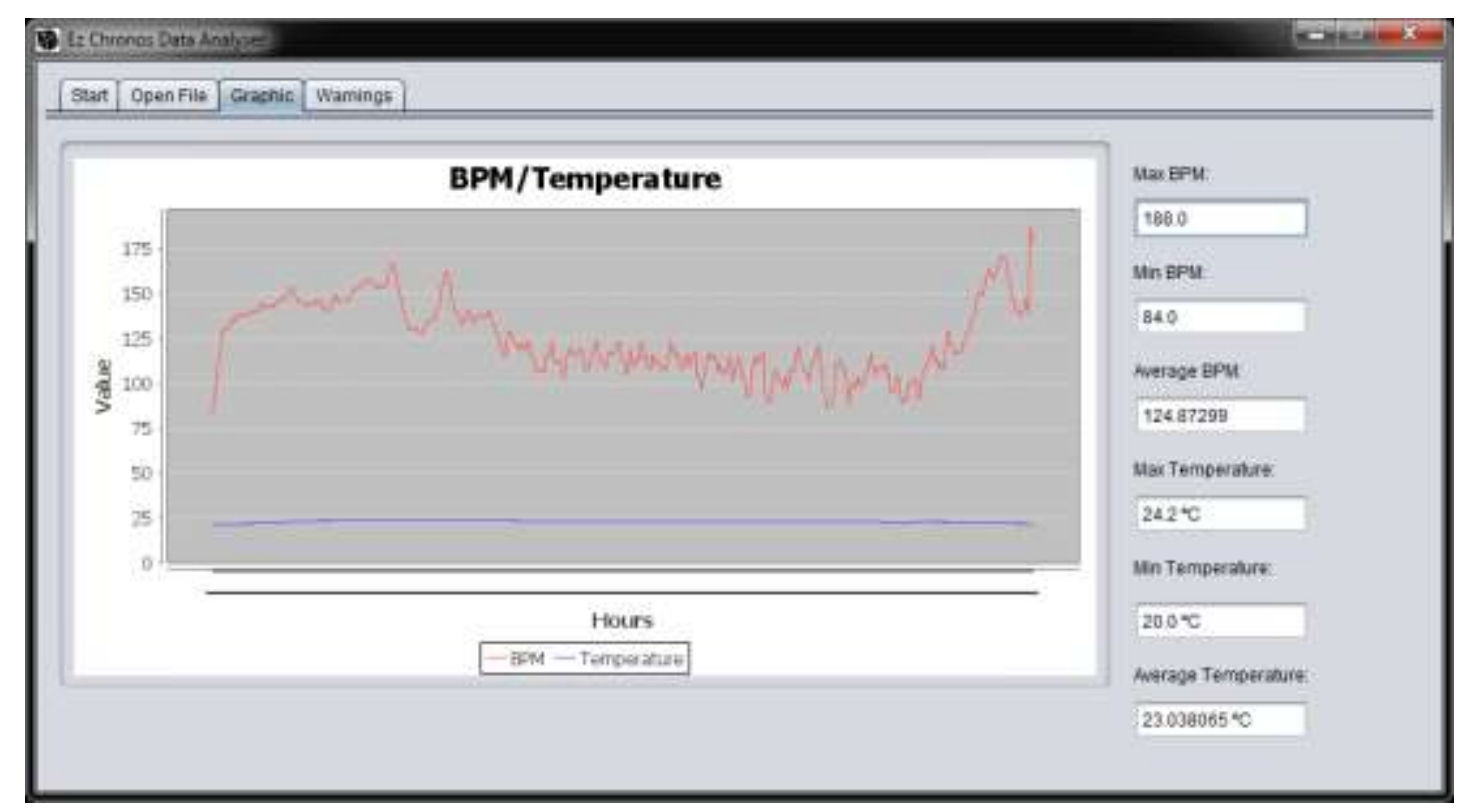

Figure 29 - Example of Graph Application and respective Information.

The analysis of risk situations has two different options: one filter for a BPM or temperature value range can be used, showing these values to the interval in question. Moreover it is provided a system analysis given information from the patient regarding his/her type of activity. We considered five levels of activity stress and took into account the patient's age: from people with minimal mobility, like bedridden, to people with high performance activities like sports for example. There is also a button which clears the data in this separator, allowing a new configuration of the alert system (Figure 30). 


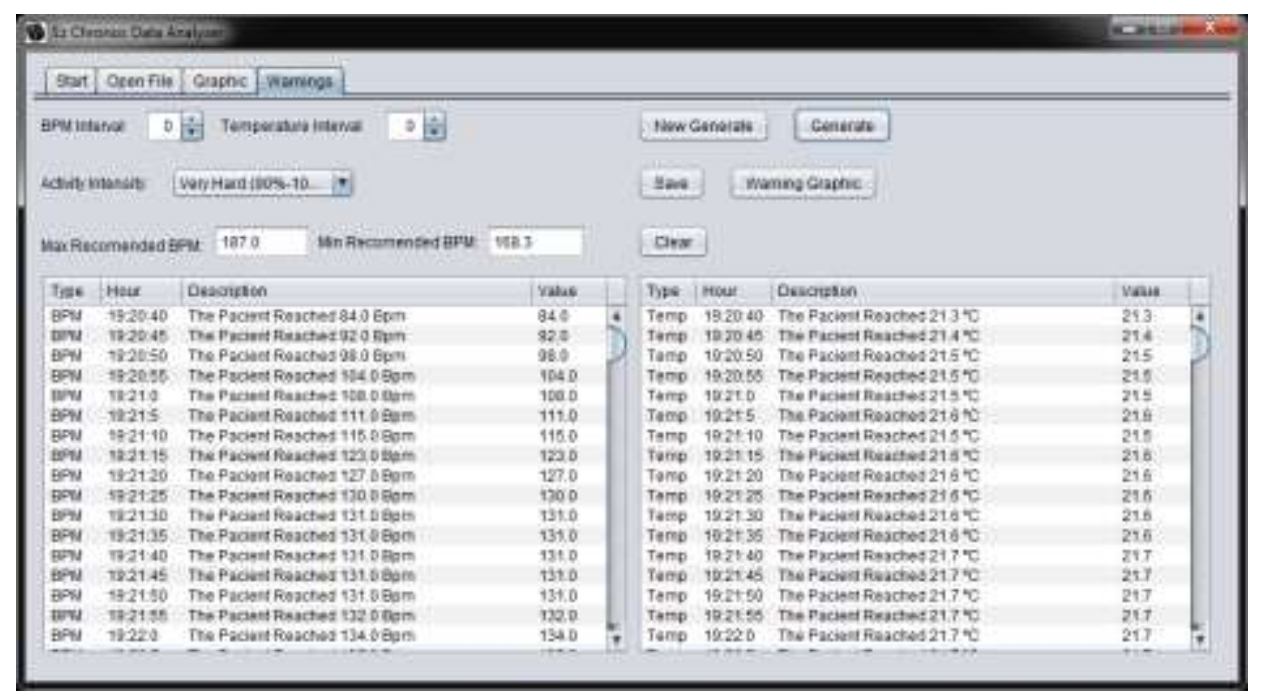

Figure 30 - Example of a data filter through the intensity level.

Finally the data can be displayed on a chart or other, containing the stored personal information of the patient as well as the respective alert.

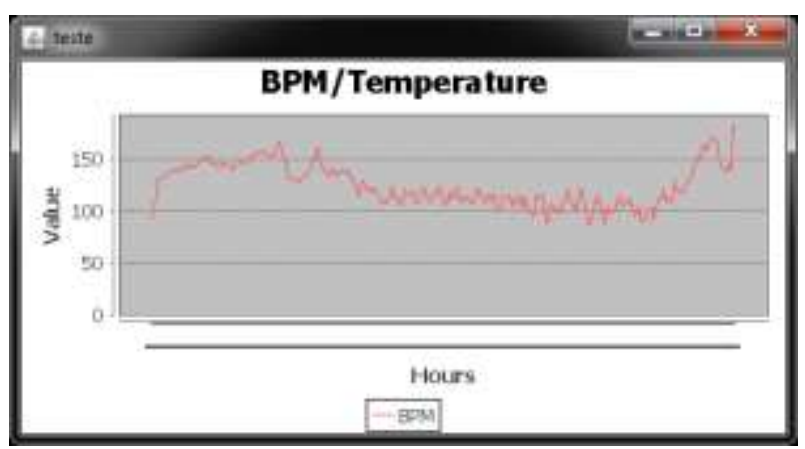

Figure 31 - Warning graphics.

\subsection{Falls Detection with External Alerts}

The falls detection system works by measuring the user's body acceleration, receiving acceleration values in real time from accelerometer and then converting those values in $G$ force. If that $G$ value exceeds $2.5 G$ a fall is detected. At this moment the device launches a notification that is automatically displayed on the main screen and a beep notifies users. After that, some patient information will be sent, like the patients location to the caretaker or emergency. 
Figure 32 shows the values captured by graphics and we can see the accelerometer in a time interval where a fall is detected and confirmed, with a red triangle signal at the bottom right of the image.

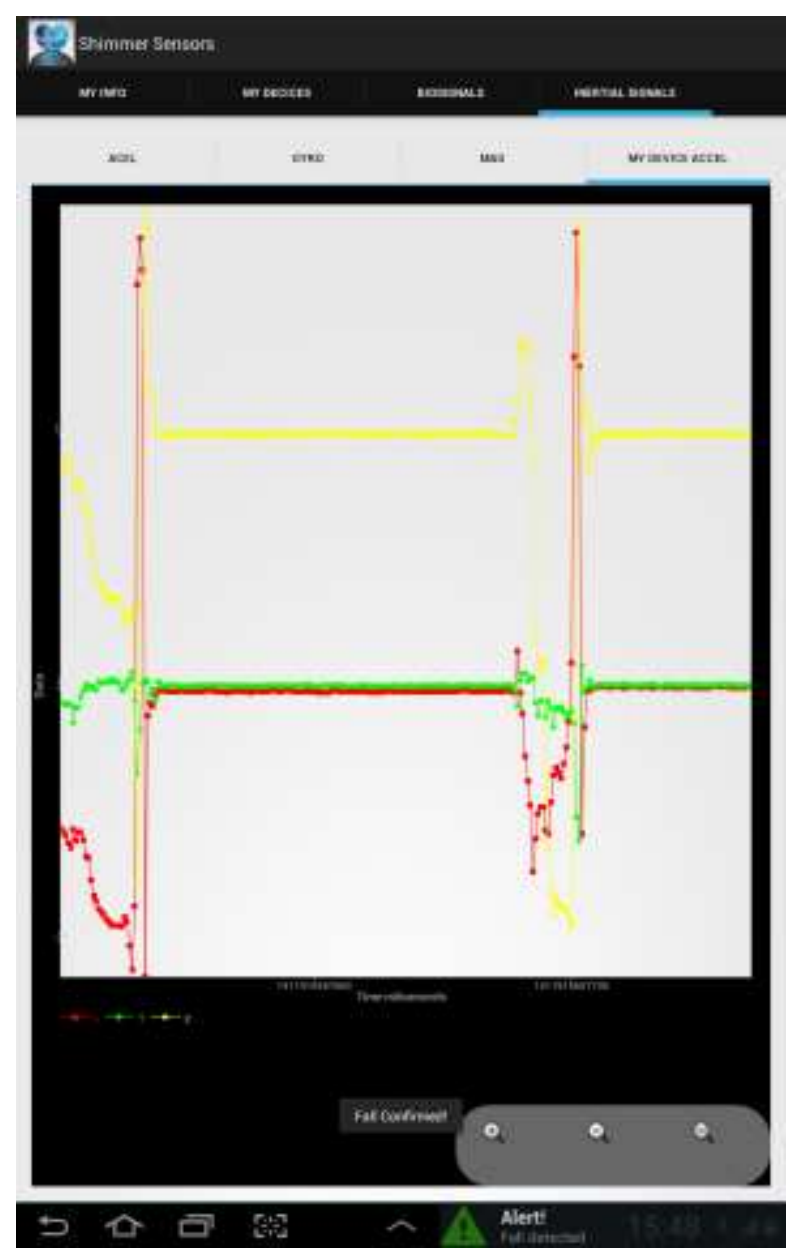

Figure 32- User Falls Detection Notification and red signal alert.

\subsection{Summary}

In this chapter, it is demonstrated, the AAL mobile solution in a real scenario. Architecture was described in Section 5.1 presenting all system modules for biofeedback monitoring and fall detections. Then, in Section 5.2, it was showed the graphical interface proper for elderly people. Section 5.3 and 5.4 performed and explained the biofeedback monitoring tools with all features and falls detection techniques. 
Both presented architectures have common goals concerning the detection and analysis of bio-signals and inertial signals. They are responsible for detecting significant changes caused by common daily activities. These collected values can also infer significant changes of data through the sensors placed along the patients' body and are able to guarantee the well-being of the elderly. It considers the interventions that a qualified healthcare team may find adequate for each user. So, there are two ways for collecting and storing data, either on-line or offline. Whether it is performed on-line (in real-time) or off-line, later analysis points to the energetic efficiency of the various devices without compromising the reliability of biofeedback collected data. 


\section{System Validation and Performance Evaluation}

This section presents the system demonstration and performance evaluation of the AAL mobile solutions developed. First, in Section 6.1 obtained results are presented considering two approaches for collecting biomedical data: the Texas Instruments programmable watch, and Shimmer sensors. Three levels of observation where considered: low for patients that are experiencing "low" levels of biomedical changes; "normal" for regular patients; and “critical” level for real-time monitoring [96].

For these solutions several trials are performed and different experimental approaches are used in order to obtain success (accuracy). The demonstration and performance evaluation included five volunteers, aged from 20 to 30 years with no previous health records information. A set of experiments has been carried out to evaluate the system performance considering the three levels of surveillance. For each value of the above mentioned levels a total of 30 trials were conducted and distributed in three levels of alerts. On these trials data was collected and stored (a total of 22 thousands samples). Subsequently, in section 6.2 those volunteers were tested by the falls detection, therefore several fall simulations were performed in order to evaluate and demonstrate the solution in a real trial scenario.

Finally, the results are deliberated for both approaches, concluding with an overall evaluation of the proposed solutions. 


\subsection{Collecting Bio-signals and their Analysis}

This developed application presented in Figure 33 gathered data (bio-signals) from one of the many experiments performed in this study. In each level, 10 samples were collected and analyzed afterwards. According to an average standard deviation of $10 \%$ and $15 \%$. The levels obtained in situations considered dangerous for the patient, were stored in a database. The graphic visualization is presented with various colors for different types of sensors.

Collecting biomedical data with the Texas Instruments programmable watch allows offline supervision at home or outdoors within a short distance. This approach was considered to be preventive or for future analysis of possible symptoms.

The volunteers did not present any complaint regarding the lack of flexibility, ergonomics, or any discomfort resulting from time extended handling and use of the chest strap. After collecting data from the watch, it is possible to analyze the same (as may be seen in Figure 33), identifying the situations of stress or major concern. It is possible to calculate average values, such as, body temperature or heart rate values. Finally, it is feasible to define warning parameters for future analysis.

Thus, a list of alert situations can be obtained and stored. At the end of the analysis, the caretaker can save the identified alerts according to the previous defined warning parameters. 


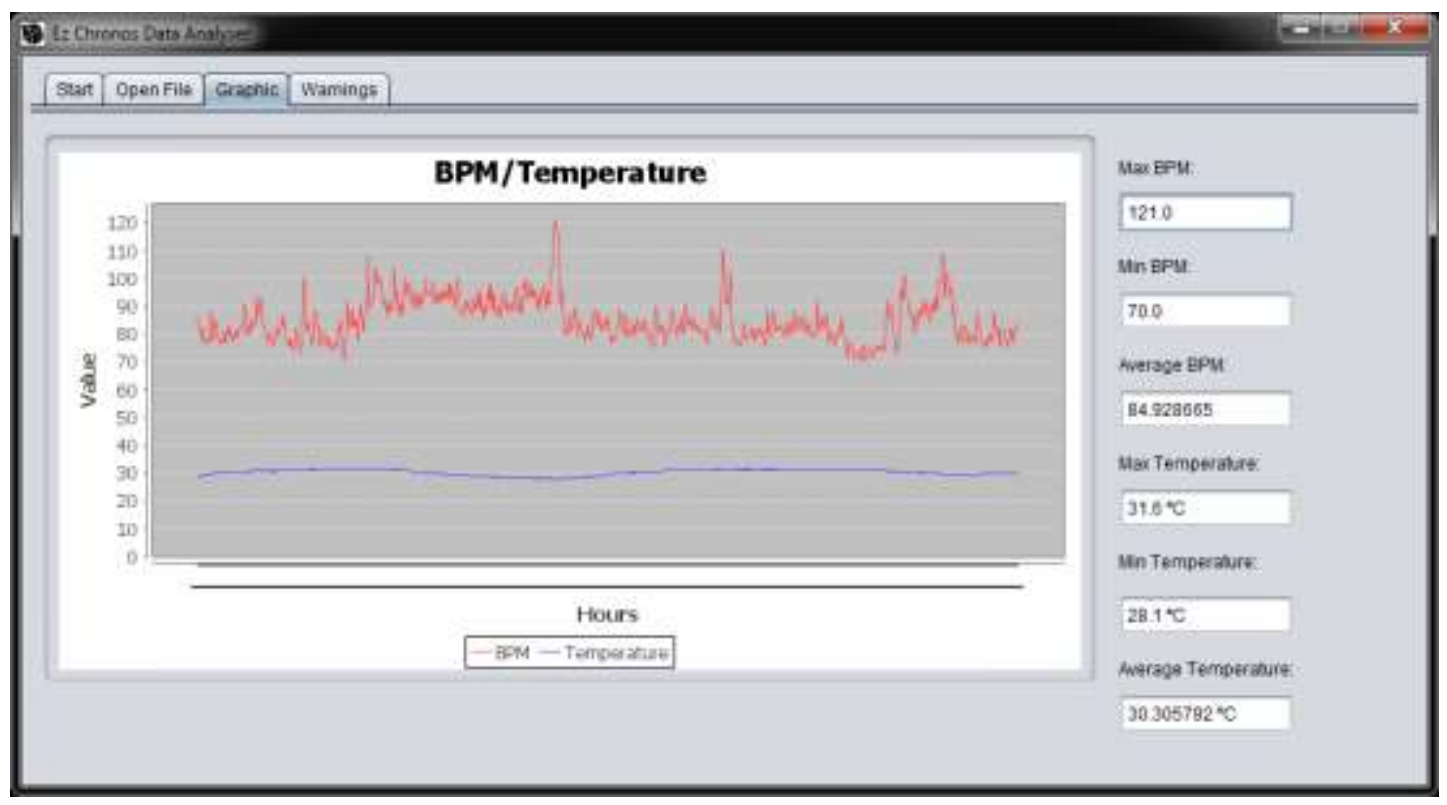

Figure 33 - Proposed application for the Texas Instruments watch showing collected data from temperature and heart rate from a given patient.

The normal monitoring level consider patients that have been recently ill or submitted to a surgery. In this context the monitoring process may occur in real time, however, with a gap time of 15 times per minute. This frequent monitoring identifies changes on the body temperature, which may easily be a signal of an infection or an alteration of heart rate. However, these changes are not considered as "high risk" situations.

The critical monitoring level considers the surveillance of the patient's health, online and in real time. The data collection must be precise to immediately identify situations that might lead to the patient's death. In this case, patients must be in a hospital or another healthcare institution followed by qualified healthcare professionals. This allows the patient to have access to medical care and information as soon as possible.

The performance evaluation of these several scenarios with the above-mentioned three levels of surveillance that considered to evaluates the performance of the proposed solution. Table 2 presents the total number of alerts per level of surveillance that show the significant differences and improvements between levels of observation. 
In the first observation level (low), volunteers were in a resting situation without making significant movements. Thus, emulating a situation of a bedridden elderly or an elderly with great physical limitations. In the second level (normal) the volunteers could have some movements performing daily tasks.

This way, representing an elderly who is able to perform some of the household chores. The third level (critical) is for cases in which patients show signs of possible risky situations. In this level, all the variations of biomedical signals are considered dangerous for the well-being and for the health of the elderly.

\begin{tabular}{c|c|c|c|c} 
& \multicolumn{2}{|c|}{ Texas Instruments Watch } & \multicolumn{2}{c}{ Shimmer Sensors } \\
& Level 1 (10\%) & Level 2 (15\%) & Level 1 (10\%) & Level 2 (15\%) \\
Low & 64 & 39 & 32 & 1 \\
Normal & 473 & 170 & 134 & 53 \\
Critical & 1754 & 788 & 504 & 250
\end{tabular}

Table 2. Total Number of Alerts Per Level of Surveillance (low, normal and critical) with a standard deviation of $10 \%$ and $15 \%$ considering the ti watch and shimmer sensors, for the 30 experiments.

The Shimmer sensors include little alerts, presenting more accurate data resulting in an error reduction when increasing the standard deviation. Moreover, when alerts occurred, their time analysis was also important. When there is an alert in only one of the samples (in time), it is not considered meaningful. The alerts in one sample can be a misreading and it is only considered as a meaningful and dangerous situation after several consecutive alerts. 


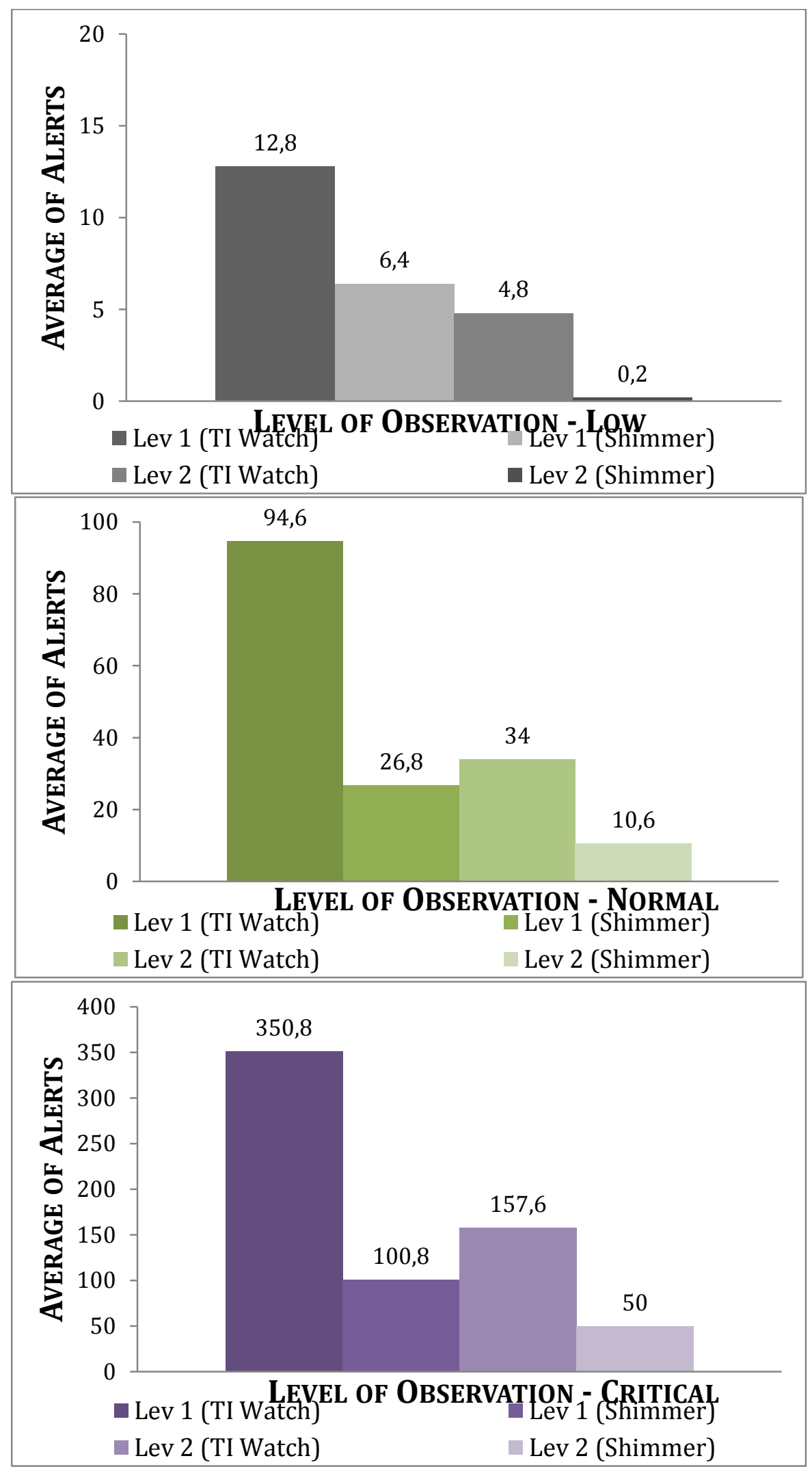

Figure 34 - Comparison of average alerts values to each level of observation system. (Watch and Shimmer) 
Figure 34 presents the comparison of average alert values to each level of observation. With the decrease and exclusion of possible errors, the results improve about $2 \%$, for the Texas Instruments watch and about $0.8 \%$ for the Shimmer sensors.

Furthermore, it is observed that various levels of observation have different amounts of alerts increasing the surveillance level. The Shimmer sensors have more capacity to read data in equal time intervals and in a worst-case scenario.

\subsection{Falls Detection and their Analysis}

The mobile application was deployed a specific functionality: fall detection in order to validate the proposed solution. The volunteer's performance several fall simulations, each one with 10 trials, in a total of 50 trials and the obtained results thought Table 3.

\begin{tabular}{c|c|c|c|c} 
Action & Number of trials & Trials errors & Right Trials & Average \\
$1^{\circ}$ Trial Fall Simulation & 50 & 7 & 43 & $86 \%$ \\
$2^{\circ}$ Trial Fall Simulation & 50 & 4 & 46 & $92 \%$
\end{tabular}

Table 3. Experimental Results Control Table.

After a total of 50 trials the conditions to identify a fall was ascertained because the interval between the initial acceleration and the identification without movement was too extensive (Figure 35). 


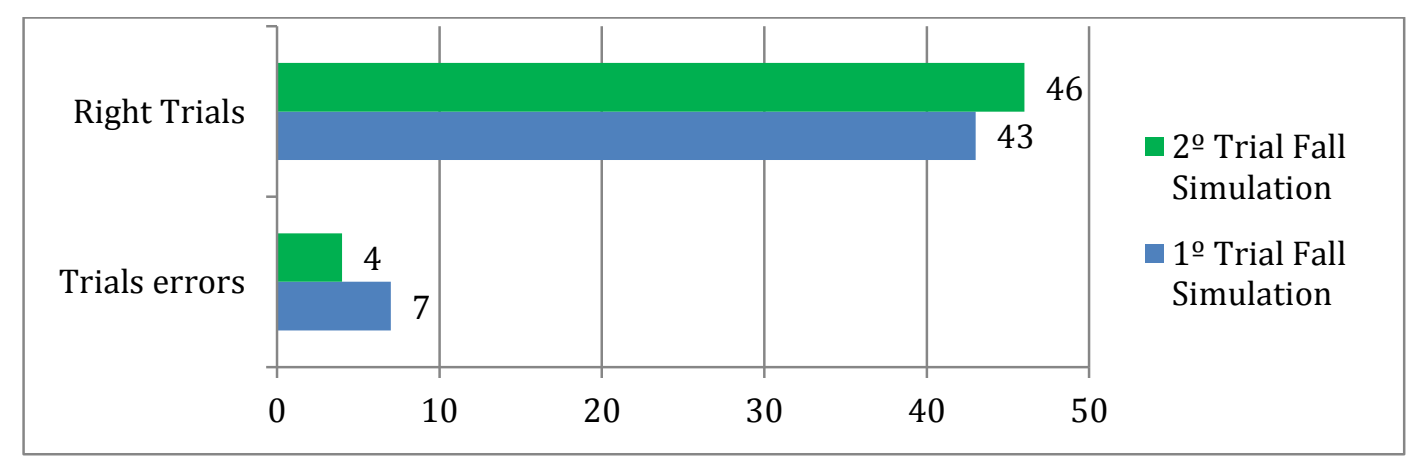

Figure 35 - Bar Chart Values Ordered.

This way the time of response was reduced and after the achievement of new trial under the same conditions, the results are improved about six percent (Figure 36).

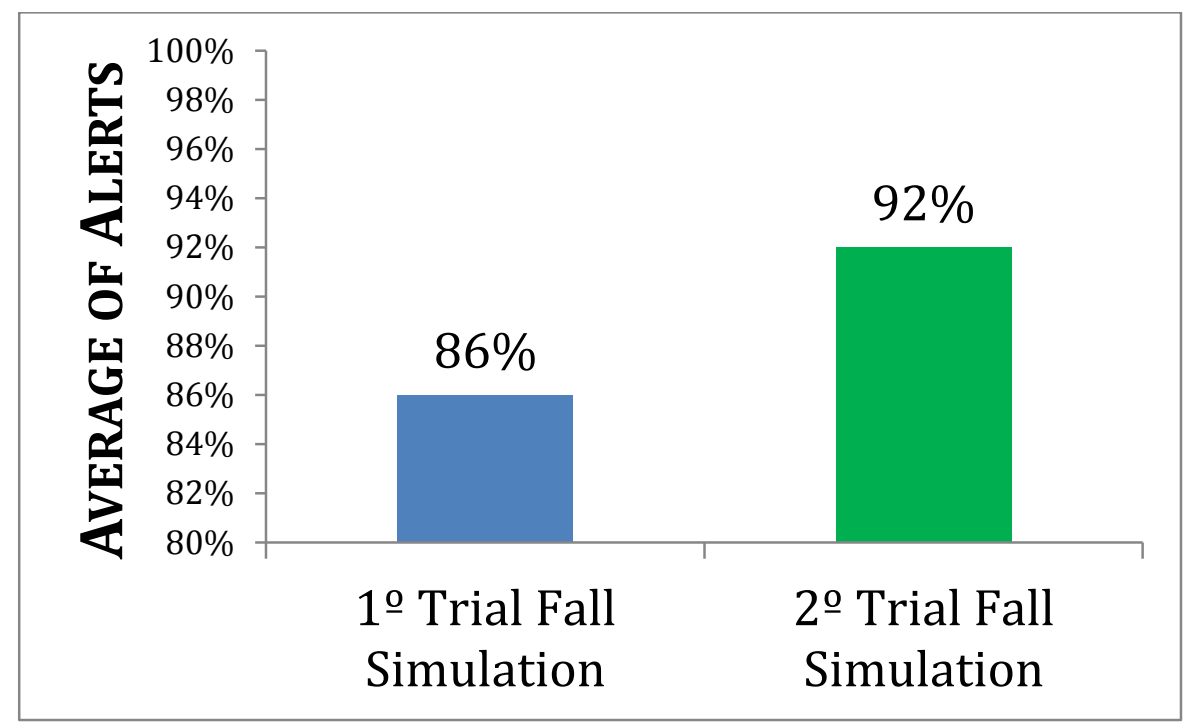

Figure 36 - Comparison of average alerts values to each simulation.

Results show a better fall detection, as expected when the time between the initial acceleration and the identification without movement was reduced, bringing improvements in real scenarios, such as one fall detected because the patient is unconscious or has suffered a heart attack. 


\subsection{Summary}

In this section the volunteers demonstrated the solution in a real experimental scenario. In section 6.1 the AAL mobile application obtained results considering two approaches for collecting biomedical data. In section 6.2 there were performed several falls simulations with those methodologies laid proofed.

Those proposals allow a customizable and a non-invasive monitoring of ECG and temperature for a patient, making his/her daily life safer and with provided benefits. Besides, it has the advantage of having a daily control in which all the information (numerical and graphical) is stored for future analysis whenever necessary. Therefore it is possible to recognize situations in that the patient health is in danger, online and in real time or by monitoring events offline. This way, it is possible to identify situations of disease, through body temperature alterations; high heart rates; abnormal rhythm detection; situations of cardiac arrhythmias and many other health situations that can be diagnosed.

Results clearly show that the presented proposal alerts immediately both elderly and caregivers of significant changes and life threatening situations. This data can identify, for example, flu symptoms, heart diseases, such as the possible beginning of cardiac problems. 


\section{Conclusions and Future}

\section{Work}

This chapter presents a synthesis of this dissertation also the main accomplishments that result from this work, with always a few words to describe one year of hard work and dedication. Moreover it is pointed out some paths for further work.

\subsection{Conclusions}

The main objective of this dissertation it to present a mobile healthcare solution for AAL environments on m-Health and with this improve the life's of elderly people that live alone or in remote locations, through several mechanisms as biofeedback monitoring tool and falls detection.

This system is prepared for continuous assistance and monitoring of bio-signals, collecting data and processing a large amount of collected information to identify potential diseases or health risk situations. Those two prototypes have a good battery lifetime and are very portable.

The eZ430-Chronos watch was used for prevention in an online and offline mode. According to the online analysis, Shimmer sensors are, no doubt, an improvement allowing the collection of data within seconds. Mobile devices, such as, smartphones or tablets were considered to send 
alerts in case of critical situations, through data transmitted from various sensors and for mobility purposes and this way identifying a stroke, falls and other dangerous incidents.

The solution offers a good and ergonomic visualization because the patient can see the data information about his/her bio-signals, inertialsignals and location where the information is displayed graphically and numerically, all this in real-time. Further it is also customizable, because it is possible to choose independently the sensors or activate only the required sensors. All this modules are shown in system architecture, as so a fall detection and real-time location.

Nowadays is almost normal for a person to have a mobile device as the consumption of mobile devices has been growing exponentially, so one can try and take maximum advantage of these devices, for they are more compact, and allow freedom of movements. This proposed solution is extremely easy to place on the patient's body, flexible, and with no harm to his/her health.

The proposed solution was evaluated and demonstrated and it is ready to use. It is a pretty good bet to aid in ordinary tasks, and much more, becoming a "healthier" tool.

Unfortunately the technology cannot detect all the falls, because there is exist a gap to find appropriate methodologies for each sensor.

System demonstration and performance evaluation is difficult to perform but during rehearsals more precise results were succeeded and one increased the exclusion of possible error results. This way it is possible to apply it in real world especially in the AAL situation where the non-stop monitoring is a key.

All this has been possible to obtain by the Texas Instrument watch, and the Shimmer sensors respectively. 


\subsection{Future Work}

To conclude this work, leaving some considerations to future developments and research based on it:

- The development and update of the APPs and APIs for other mobile platforms, such as iOS from Apple and Windows Phone.

- Furthermore, the data retrieved from the other sensors for example Electroencephalography (EEG) beyond the aforementioned ECG, EMG, and accelerometer to accomplish better falls detection or prevention.

- From a smartphone or tablet create a "health sensor" and become a specific device to resolve security issues.

- Join two more types of sensors to analyse the patient's posture that will add a great value and a wider meticulousness on healthcare assistance to the elderly.

- A performance assessment study through simulation considering different danger scenarios and evaluate the scalability of the proposed solution, may also be considered.

All these proposed improvements focusing in the elderly without forgetting compromising the flexibility of movements and autonomy or security. 



\section{References}

[1] Ageing and life course, World Health Organization, [Online]. Available: http://www.who.int/ageing/en/ ; Accessed: October, 2013.

[2] Eurostat, Demography Report 2010 - Older, more numerous and diverse Europeans, Publications Office of the European Union, 2011. http:/ / ec.europa.eu/social/main.jsp?catld=738\&langld=en\&publd=593 6\&furtherPubs=yes

[3] World Health Organization, “Disability and health", http://www.who.int/mediacentre/factsheets/fs352/en/index.html, June 2011.

[4] U.S. Department of Health and Human Services, "Profile of Older Americans: 2011", http://www.aoa.gov/Aging_Statistics/Profile/2011/docs/2011profile.p df, 2011.

[5] Tomoya Shimokakimoto, K. Suzuki, "A chair-type interface for longterm and ambient vital sensing", Annual International Conference of the IEEE, Engineering in Medicine and Biology Society (EMBC 2011), pp. 1173-1176, Aug. 30 - Sept. 3, 2011. 
[6] Venkatesh, V.; Vaithyanathan, V.; Manikandan, B.; Raj, P., "A Smart Ambulance for the synchronized health care - A service oriented device architecture-based," Computer Communication and Informatics (ICCCI), 2012 International Conference, pp.1-6, 10-12 Jan., 2012.

[7] Roham, M.; Saldivar, E.; Raghavan, S.; Zurcher, M.; Mack, J.; Mehregany, M., "A mobile wearable wireless fetal heart monitoring system," Medical Information \& Communication Technology (ISMICT), 2011 5th International Symposium, pp.135-138, 27-30 March, 2011.

[8] McNaull, J.; Augusto, J.C.; Mulvenna, M.; McCullagh, P., "Multi-agent Interactions for Ambient Assisted Living," Intelligent Environments (IE), 2011 7th International Conference, pp.310-313, 25-28 July, 2011.

[9] Rodrigues, A.; Resende, C.; Carvalho, L.; Saleiro, P.; Abrantes, F., "Performance analysis of an adaptable home healthcare solution," eHealth Networking Applications and Services (Healthcom), 2011 13th IEEE International Conference, pp.134-141, 13-15 June, 2011.

[10] World Health Organization, “Disability and health", http://www.who.int/mediacentre/factsheets/fs352/en/index.html, June 2011.

[11] Ziefle, M.; Rocker, C., "Acceptance of pervasive healthcare systems: A comparison of different implementation concepts," Pervasive Computing Technologies for Healthcare (PervasiveHealth), 2010 4th International Conference on-NO PERMISSIONS, pp.1-6, 22-25 March, 2010.

[12] McNeely E. The consequences of job stress for nurses' health: time for a check-up. Nursing Outlook, 2005. 
[13] Sterritt, Roy; Nugent, C., "Autonomic Computing and Ambient Assisted Living - Extended Abstract," Engineering of Autonomic and Autonomous Systems (EASe), 2010 Seventh IEEE International Conference and Workshops, pp.149-151, 22-26 March, 2010.

[14] Alerndar, H.; Ertan, H.; Incel, O.D.; Ersoy, C., "ARAS human activity datasets in multiple homes with multiple residents," Pervasive Computing Technologies for Healthcare (PervasiveHealth), 2013 7th International Conference, pp.232-235, 5-8 May, 2013.

[15] Schepps, J, Rosen A, “Microwave industry outlook - wireless communications in healthcare". Microwave Theory and Techniques vol. 50, pp 1044-1045, Mar., 2002.

[16] Hong Sun, V.De Florio, Ning Gui, C. Blondia, "Promises and Challenges of Ambient Assisted Living Systems," Information Technology: New Generations, 2009. ITNG '09. Sixth International Conference, pp.12011207, 27-29 April, 2009.

[17] PERSONA EU-project. [Online]. Available: http://www.aalpersona.org/ ; Accessed: September, 2013.

[18] Huertas, S.; Lázaro, J.P.; Guillen, S.; Traver, V., "Information and assistance bubbles to help elderly people in public environments," Engineering in Medicine and Biology Society (EMBC), 2010 Annual International Conference of the IEEE,pp.208-211, Aug. 31-Sept. 4, 2010.

[19] Lázaro, J.P.; Fides, A.; Navarro, A.; Guillen, S., "Ambient Assisted Nutritional Advisor for elderly people living at home," Engineering in Medicine and Biology Society (EMBC), 2010 Annual International Conference of the IEEE, pp.198-203, Aug. 31-Sept. 4, 2010. 
[20] M. A. Estudillo-Valderrama, L. M. Roa, J. Reina-Tosina, I. RománMartínez, "Ambient Assisted Living: A methodological approach," Engineering in Medicine and Biology Society (EMBC 2010), Annual International Conference of the IEEE, pp.2155-2158, August 31September 4, 2010.

[21] Kofod-Petersen, A., "Minimising Information Asymmetry by Using Proxies," Complex, Intelligent and Software Intensive Systems (CISIS), 2010 International Conference, pp.878-882, 15-18 Feb., 2010.

[22] Q. Jones and S. A. Grandhi, "P3 systems: Putting the place back into social networks," IEEE Internet Computing, vol. 9, pp. 38-46, 2005.

[23] Edwards, C., "Wearable computing struggles for social acceptance," IEE Review, vol.49, no.9, pp.24-25, Sept., 2003.

[24] Unger, S.; Pfeiffer, S.; Timmermann, D., "Dethroning Transport Layer Security in the Embedded World," New Technologies, Mobility and Security (NTMS), 2012 5th International Conference, pp.1-5, 7-10 May, 2012.

[25] Amirjavid, Farzad; Bouzouane, Abdenour; Bouchard, Bruno, "Intelligent temporal data driven world actuation in ambient environments: Case study: Anomaly recognition and assistance provision in smart home," Computer and Information Science (ICIS), 2013 IEEE/ACIS 12th International Conference, pp.287-293, 16-20 June, 2013.

[26] M. M. Sohlberg, B. Todis, S.Fickas, P.F. Hung, R. Lemoncello, "A profile of community navigation in adults with chronic cognitive impairments”, Brain Inj., vol 19, no. 14, pp. 1249-1259, 2005. 
[27] Schaub, F.; Konings, B.; Weber, M.; Kargl, F., "Towards context adaptive privacy decisions in ubiquitous computing," Pervasive Computing and Communications Workshops (PERCOM Workshops), 2012 IEEE International Conference, pp.407-410, 19-23 March, 2012.

[28] Klein, B.; Reutzel, S.; Rossberg, H.H.; Cook, G., "Can telecare contribute to an independent life at home with 100? A glance to the UK and initial experiences of the German LOEWE field test on age appropriate sensor based assistance in real estate," Human System Interaction (HSI), 2013 The 6th International Conference, pp.594-599, 6-8 June, 2013.

[29] Hervas, R.; Bravo, J.; Fontecha, J., "An assistive navigation system based on augmented reality and context awareness for people with mild cognitive impairments," Biomedical and Health Informatics, IEEE Journal, vol.18, pp.368-374, Jan., 2014.

[30] Busching, F.; Bottazzi, M.; Wolf, L., "The GAL monitoring concept for distributed AAL platforms," e-Health Networking, Applications and Services (Healthcom), 2012 IEEE 14th International Conference, pp.466-469, 10-13 Oct., 2012.

[31] Continua Health Alliance, [Online]. Available: http://www.continuaalliance.org/ ; Accessed: October, 2013.

[32] Baquero S, R.; Rodriguez G, J.G.; Mendoza C, S.; Decouchant, D., "Towards a uniform sensor-handling scheme for Ambient Intelligence systems," Electrical Engineering Computing Science and Automatic Control (CCE), 2011 8th International Conference, pp.1-6, 26-28 Oct., 2011. 
[33] E. Foxlin, "Pedestrian tracking with shoe-mounted inertial sensors," IEEE Computer Graphics and Applications, vol. 25, no. 6, pp.38-46, Dec., 2005.

[34] A. Marcus, G. Davidzon, D. Law, N. Verma, R. Fletcher, A. Khan, and L. Sarmenta, "Using NFC-enabled Mobile Phones for Public Health in Developing Countries," in Proc. IEEE Conf. Near-Field Communication (NFC), 2009.

[35] Figueiredo, C.P.; Gama, O.S.; Pereira, C.M.; Mendes, P.M.; Silva, S.; Domingues, L.; Hoffmann, K. -P, "Autonomy Suitability of Wireless Modules for Ambient Assisted Living Applications: WiFi, Zigbee, and Proprietary Devices," Sensor Technologies and Applications (SENSORCOMM), 2010 Fourth International Conference, pp.169-172, 1825 July, 2010.

[36] Leusmann, P.; Mollering, C.; Klack, L.; Kasugai, K.; Ziefle, M.; Rumpe, B., "Your Floor Knows Where You Are: Sensing and Acquisition of Movement Data," Mobile Data Management (MDM), 2011 12th IEEE International Conference, pp.61-66, 6-9 June, 2011.

[37] P.Khanja, S.Wattanasirichaigoon, J.Natwichai, L.Ramingwong, and S.Noimanee, “A Web Base System for ECG Data Transfered using ZigBee/IEEE Technology", 3rd International Symposium on Biomedical Engineering, 2008.

[38] K. Shimizu, "Telemedicine by mobile communication," IEEE Engineering in Medicine and Biology Magazine, vol. 18, no. 4, pp. 3244, 1999.

[39] Ferguson, G. T., “Have Your Objects Call My Objects". In: Harvard Business ReView, Vol. 80, No.6, pp. 138 - 143, 2003. 
[40] Gellersen, H.-W.; Schmidt, A; Beigl, M., "Adding some smartness to devices and everyday things," Mobile Computing Systems and Applications, 2000 Third IEEE Workshop in Monterrey, USA, pp.3-10, 2000.

[41] Cascado, D.; Romero, S.J.; Hors, S.; Brasero, A.; Fernandez-Luque, L.; Sevillano, J.L., "Virtual worlds to enhance Ambient-Assisted Living," Engineering in Medicine and Biology Society (EMBC), 2010 Annual International Conference of the IEEE , pp.212-215, Aug. 31 - Sept. 4, 2010.

[42] Virtual Worlds, Second Life, [Online]. Available: http://secondlife.com ; Accessed: December, 2013.

[43] Gorini, A., Gaggioli, A., et al., “A second life for ehealth: Prospects for the use of 3-d virtual worlds in clinical psychology". J Med Internet Res, vol 10(3), 2008.

[44] M. J, “Internet 3.0: the internet of things," Analysys Mason, Tech. Rep., 2010.

[45] Dohr, A.; Modre-Opsrian, R.; Drobics, M.; Hayn, D.; Schreier, G., "The Internet of Things for Ambient Assisted Living," Information Technology: New Generations (ITNG), 2010 Seventh International Conference on, pp.804-809, 12-14 April, 2010.

[46] Schaar, AK.; Ziefle, M., "Smart clothing: Perceived benefits vs. perceived fears," Pervasive Computing Technologies for Healthcare (PervasiveHealth), 2011 5th International Conference, pp.601-608, 2326 May, 2011. 
[47] Doukas, C.; Maglogiannis, I, "Managing Wearable Sensor Data through Cloud Computing," Cloud Computing Technology and Science (CloudCom), 2011 IEEE Third International Conference, pp.440-445, Nov. 29 -Dec. 1, 2011.

[48] Coyle, S.; Lau, K.-T.; Moyna, N.; O'Gorman, D.; Diamond, D.; Di Francesco, F.; Schoumacker, S.; Mourier, V.; Chartier, I; Convert, R.; De-Moncuit, H.; Bini, C., "BIOTEX-Biosensing Textiles for Personalised Healthcare Management," Information Technology in Biomedicine, IEEE Transactions, vol.14, pp.364-370, March, 2010.

[49] M. Fukumoto and Y. Tonomura, "Body coupled fingering: Wireless wearable keyboard," in Proc. Conf. Human Factors Comp. Syst. (CHI), pp. 147-154, 1997.

[50] T. Starner, J. Auxier, D. Ashbrook and M. Gandy "The Gesture Pendant: a self-illuminating, wearable, infrared computer vision system for home automation control and medical monitoring", Proc. ISWC, 2000.

[51] Scheermesser, M.; Kosow, H.; Rashid, A; Holtmann, C., "User acceptance of pervasive computing in healthcare: Main findings of two case studies," Pervasive Computing Technologies for Healthcare, 2008. PervasiveHealth 2008. Second International Conference, pp.205-213, Jan. 30 -Feb. 1, 2008.

[52] Megalingam, Rajesh Kannan; Radhakrishnan, V.; Jacob, D.C.; Unnikrishnan, D.K.M.; Sudhakaran, AK., "Assistive Technology for Elders: Wireless Intelligent Healthcare Gadget," Global Humanitarian Technology Conference (GHTC), 2011 IEEE, pp.296-300, Oct. 30-Nov. 1, 2011. 
[53] H. Noshadi, S. Ahmadian, H. Hagopian, J. Woodbridge, F. Dabiri, N. Amini, M. Sarrafzadeh, N. Terrafranca, "HERMES - Mobile Balance and Instability Assessment System," Proc. of the International Conference on Bio-inspired Systems and Signal Processing (BIOSIGNALS 2010), Valencia, Spain, pp. 264-270, Jan., 2010.

[54] Carswell, W.; Augusto, J.; Mulvenna, M.; Wallace, J.; Martin, S.; McCullagh, P.J.; Zheng, H.; Wang, H.; McSorley, K.; Taylor, B.; Jeffers, W. P., "The NOCTURNAL Ambient Assisted Living system," Pervasive Computing Technologies for Healthcare (PervasiveHealth), 2011 5th International Conference, pp.208-209, 23-26 May, 2011.

[55] Linti, C., Horter, H., Osterreicher, P., Planck, H. (2006). “Sensory Baby Vest for the Monitoring of Infants". Proceedings of the International Workshop on Wearable and Implantable Body Sensor Networks, Cambridge, MA, pp. 135 - 137, April 3 - 5, 2006.

[56] J. Sousa, S. Palma, H. Silva, and H. Gamboa, “aal@home: a New Home Care Wireless Biosignal Monitoring Tool for Ambient Assisted Living," in Proc. INSTICC - 1st International Living Usability Lab Workshop on AAL Latest Solutions, Trends and Applications, Rome, Italy, Jan., 2011.

[57] Dhillon, M.S.; McCombie, S.A; McCombie, D.B., "Towards the prevention of pressure ulcers with a wearable patient posture monitor based on adaptive accelerometer alignment," Engineering in Medicine and Biology Society (EMBC), 2012 Annual International Conference of the IEEE, pp.4513-4516, Aug. 28 -Sept. 1, 2012.

[58] Robert J. Orr and Gregory D. Abowd "The smart floor: A mechanism for natural user identification and tracking" to appear in the Proceedings of the 2000 Conference on Human Factors in Computing Systems (CHI 2000), The Hague, Netherlands, April 1-6, 2000. 
[59] Kondo, K.; Kanegae, M.; Koizumi, T.; Obata, K.; Nakamura, Y., "Memory Ubiquitous: Providing Memories on Anything, Anywhere - A Case Study for Cooking Support," Multimedia (ISM), 2010 IEEE International Symposium, pp.260-265, 13-15 Dec., 2010.

[60] M. Chan, D. Esteve, C. Escriba, and E. Campo, "A review of smart homes- present state and future challenges.," Computer Methods and Programs in Biomedicine, vol. 91, pp. 55-81, Jul, 2008.

[61] Fortino, G.; Gravina, R.; Guerrieri, A., "Agent-oriented integration of body sensor networks and building sensor networks," Computer Science and Information Systems (FedCSIS), 2012 Federated Conference on, pp.1207-1214, 9-12 Sept., 2012.

[62] IBM, TheSmarterCity, [Online]. Available: http://www304.ibm.com/easyaccess/cpe/html0/186287.html ;/; Accessed: May, 2014.

[63] Johan H. Huijsing, Smart Sensor Systems: Why? Where? How? In Gerard C. M. Meijer, Ed., Smart Sensor Systems, Wiley, 2008.

[64] Frey, J., "AdAPT - A Dynamic Approach for Activity Prediction and Tracking for Ambient Intelligence," Intelligent Environments (IE), 2013 9th International Conference, pp.254-257, 16-17 July, 2013.

[65] Valente, P.; Hossain, S.; Gronbak, B.; Hallenborg, K.; Reis, L.P., "A multi-agent framework for coordination of intelligent assistive technologies," Information Systems and Technologies (CISTI), 2010 5th Iberian Conference, pp.1-6, 16-19 June 2010, and in https://sites.google.com/site/prvalente/project-description, 2010. 
[66] Merico, D.; Bisiani, R.; Ali, H., "Demonstrating Contexta-CARE: A situation-aware system for supporting independent living," Pervasive Computing Technologies for Healthcare (PervasiveHealth), 2013 7th International Conference, pp.309-310, 5-8 May, 2013.

[67] Sikora, A., "Wireless network and gateway architectures for home care applications," Intelligent Data Acquisition and Advanced Computing Systems (IDAACS), 2011 IEEE 6th International Conference, vol.2, pp.832-836, 15-17 Sept. 2011, and in http://www.incasaproject.eu/news.php, 2011.

[68] Tiadjio, A.M.; Jamboti, K., "Requirements and Evaluation of Safety Analysis Techniques for Ambient Assisted Living Systems," Software Reliability Engineering Workshops (ISSREW), 2012 IEEE 23rd International Symposium, pp.319-324, 27-30 Nov., 2012.

[69] [30] Tiadjio, A.M.; Jamboti, K., "Requirements and Evaluation of Safety Analysis Techniques for Ambient Assisted Living Systems," Software Reliability Engineering Workshops (ISSREW), 2012 IEEE 23rd International Symposium, pp.319-324, 27-30 Nov., 2012.

[70] Zeli Gao, Jie Wu, Jianli Zhou, Wei Jiang, Lihui Feng, "Design of ECG Signal Acquisition and Processing System," Biomedical Engineering and Biotechnology (iCBEB), 2012 International Conference, pp.762-764, 2830 May, 2012.

[71] Kanoun, K.; Mamaghanian, H.; Khaled, N.; Atienza, D., "A real-time compressed sensing-based personal electrocardiogram monitoring system," Design, Automation \& Test in Europe Conference \& Exhibition, 2011, pp.1-6, 14-18 March, 2011. 
[72] K.V. Suarez, J.C. Silva, Y.Berthoumieu, P. Gomis, N. Mohamed, "ECG Beat Detection Using a Geometrical Matching Approach," Biomedical Engineering, IEEE Transactions, vol.54, no.4, pp.641-650, April 2007.

[73] Chul-Gyu Song; Keo-Sik Kim; Yang-Su An; Jeong-Hwan Seo, "Comparison of bio-impedance changes and EMG activity during daily events," Biomedical Circuits and Systems Conference IEEE , (BioCAS 2008), pp.369-372, 20-22 Nov., 2008.

[74] M. Z. Poh, N. Swenson, and R. Picard, "A Wearable Sensor for Unobtrusive, Long-term Assessment of Electrodermal Activity," IEEE Trans Biomed Eng, vol. 57, pp. 1243-1252, 2010.

[75] Rabah, H.; Amira, A.; Ahmad, A., "Design and implementation of a fall detection system using compressive sensing and shimmer technology," Microelectronics (ICM), 2012 24th International Conference, pp.1-4, 1620 Dec., 2012.

[76] E. T. Horta, I. C. Lopes, J. J. P. C. Rodrigues and S. Misra, "Real time falls prevention and detection with biofeedback monitoring solution for mobile environments", 2013 IEEE 15th International Conference eHealth Networking on Applications \& Services (IEEE Healthcom 2013), Lisbon, Portugal, pp. 594-600, 9-12. Oct., 2013.

[77] Texas Instruments, “EZ430-Chronos", 2014 [Online]. Available: http://processors.wiki.ti.com/index.php/EZ430-Chronos; Accessed: May, 2014.

[78] Shimmer, "Shimmers Sensors", 2014 [Online]. Available: http://www.shimmersensing.com/; Accessed: May, 2014. 
[79] Burns, A; Greene, B.R.; McGrath, M.J.; O'Shea, T.J.; Kuris, B.; Ayer, S.M.; Stroiescu, F.; Cionca, V., "SHIMMER ${ }^{\mathrm{TM}}$ - A Wireless Sensor Platform for Noninvasive Biomedical Research," Sensors Journal, IEEE, vol.10, pp.1527-1534, Sept., 2010.

[80] Xiao-Fei Teng; Yuan-Ting Zhang; Poon, C. C Y; Bonato, P., "Wearable Medical Systems for p-Health," Biomedical Engineering, IEEE Reviews, vol.1, pp.62-74, 2008.

[81] Fan, L.; Buchanan, W.; Thummler, C.; Lo, O.; Khedim, A; Uthmani, O.; Lawson, A; Bell, D., "DACAR Platform for eHealth Services Cloud," Cloud Computing (CLOUD), 2011 IEEE International Conference, pp.219-226, 4-9 July, 2011.

[82] HealthVault, [Online]. Available: https://www.healthvault.com/pt/pt/; Accessed: May, 2014.

[83] Adibi, S., "Link Technologies and BlackBerry Mobile Health (m-Health) Solutions: A Review," Information Technology in Biomedicine, IEEE Transactions, vol.16, pp.586-597, July, 2012.

[84] Bujnak, J.; Simsik, D.; Onofrejova, D., "Telemedicine functions in ICT social services," Applied Machine Intelligence and Informatics (SAMI), 2012 IEEE 10th International Symposium, pp.329-334, 26-28 Jan., 2012.

[85] Sánchez-Tato, I.; Senciales, J.C.; Salinas, J.; Fanucci, L.; Pardini, G.; Costalli, F.; Dalmiani, S.; de la Higuera, J.M.; Vukovic, Z.; Cicigoj, Z., "Health @ Home: A telecare system for patients with chronic heart failure," Broadband and Biomedical Communications (IB2Com), 2010 Fifth International Conference, pp.1-5, 15-17 Dec., and in http://www.health-at-home.eu/, 2010. 
[86] Jiang Jiehui; Zhang Jing, "Remote patient monitoring system for China," Potentials, IEEE , vol.26, no.3, pp.26,29, May-June, 2007.

[87] Bennebroek, M.; Barroso, A.; Atallah, L.; Lo, B.; Guang-Zhong Yang, "Deployment of wireless sensors for remote elderly monitoring," eHealth Networking Applications and Services (Healthcom), 2010 12th IEEE International Conference, pp.1-5, 1-3 July, and in http://www.continuaalliance.org/, 2010.

[88] Friedman, E.A, "Computer-assisted medical diagnosis for rural SubSaharan Africa," Technology and Society Magazine, IEEE, vol.28, no.3, pp.18,27, 2009.

[89] Object Management Group, “UML," [Online]. Available: http://www.uml.org/; Accessed: February, 2014.

[90] Google, "Android versions distribution," [Online]. Available: http://http://developer.android.com/about/dashboards/index.html ; Accessed: May, 2014.

[91] Google, "Android Software Development Kit," [Online]. Available: http://developer.android.com/sdk/index.html ; Accessed: April, 2013.

[92] Google, "Google Official Blog," [Online]. Available: http://googleblog.blogspot.co.uk/2013/03/update-from-ceo.html/ ; Accessed: April, 2014.

[93] Shimmer, "Shimmer Android ID," [Online]. Available: http://www.shimmersensing.com/shop/shimmer-android-id Accessed: April, 2013. 
[94] M. V. Ramesh, S. Anand and P. Rekha, "A mobile software for health professionals to monitor remote patients", Ninth International Conference on Wireless and Optical Communications Networks (WOCN 2012), pp. 1-4, 20-22 Sept., 2012.

[95] J. J. Oresko, J. Zhanpeng, J. Cheng, S. Huang, Y. Sun, H. Duschl and A.C. Cheng, "A Wearable Smartphone-Based Platform for Real-Time Cardiovascular Disease Detection Via Electrocardiogram Processing", IEEE Transactions on Information Technology in Biomedicine, vol. 14, pp. 734-740, May, 2010.

[96] Ramesh, M.V.; Ragi, G.R.; Abishek, T.K., "Low Power Intelligent Wearable Cardiac Sensor Using Discrete Wavelet Compression," Advances in Mobile Network, Communication and its Applications (MNCAPPS), 2012 International Conference, pp.107-110, 1-2 Aug., 2012. 



\section{Appendix}

This appendix includes the main contribution to this dissertation. The paper is entitled "A Mobile Healthcare Solution for Ambient Assisted Living Environments" and was submitted and presented at the international conference (IEEE HEALTHCOM 2014). 



\title{
A Mobile Healthcare Solution for Ambient Assisted Living Environments
}

\author{
Daniel F. M. Rodrigues ${ }^{1}$, Edgar T. Horta ${ }^{1}$, Bruno M. C. Silva ${ }^{1}$, Fábio D. M. Guedes ${ }^{1}$, and Joel J. P. C. Rodrigues ${ }^{1,2}$ \\ ${ }^{1}$ Instituto de Telecomunicações, University of Beira Interior, Covilhã, Portugal \\ ${ }^{2}$ University ITMO, Saint-Petersburg, Russia
}

daniel_f_m_rodrigues@hotmail.com; \{edgar.horta; bruno.silva\}@it.ubi.pt; fdguedes12@gmail.com; joeljr@ieee.org

\begin{abstract}
Elderly people need regular healthcare services and several times are dependent of physicians' personal attendance. This dependence rises several issues to elders, such as the need to travel and mobility support. Mobile Health (m-Health) services and applications offer good healthcare solutions that can be used indoor or in mobility environments. This paper presents an ambient assisted living (AAL) solution for mobile environments. It includes elderly biofeedback monitoring using body sensors for data collection offering support for remote monitoring. The used sensors are attached to the human body (such as the electrocardiogram, blood pressure, and temperature). They collect data providing comfort, mobility, and guaranteeing efficiency and data confidentiality. Periodic collection of patients' data is important to gather more accurate measurements and to avoid common risky situations. The presented proposal monitors elderly people, storing collected data in a personal computer, tablet, or smartphone through Bluetooth. This application allows an analysis of possible health condition warnings based on the input of supporting charts, and real-time bio-signals monitoring.
\end{abstract}

Keywords - Mobile Health; Biofeedback Monitoring; Ambient Assisted Living; Body Sensor Networks; Mobile Wearable Sensors

\section{INTRODUCTION}

In the last decade several studies are showing that elderly population is quickly increasing. It is expected that about 2 billion people will be aged 60s and older by 2050 [1]. In 2010, $17.4 \%$ of the 27 European countries' population was over 65 years old and experts foresee that, in 2060, this value will attain to $30.0 \%$ [2]. Nowadays, people has the need and social duty to assist their older parents. This aged population needs regular healthcare services and, frequently, live alone. Nearly 10 million elderly Americans over 65 years old live alone [3]. This emergent reality presents several challenges and opportunities for new and innovative healthcare services. IT solutions have taken advantage of this fact directing resources and competencies to elderly related healthcare solutions. These solutions aim to provide an assisted life style, preventing their isolation by connecting them with relatives and the community.

Nowadays, there is a significant growing market of small sensors embedded in clothing, jewelry, watches, shoes, phones, and other mobile devices like the Chair-type Interface [4] that elderly can use daily. An elderly diagnosed with dementia illness would wear these small sensors allowing the assessment of health parameters without the need to go to a medical doctor office or to a healthcare center. Therefore, it allows a better quality of life (QoL) and reduces costs for the health insurance system.

Embedded health sensors are very important because they may be able to assist people using the sensors data or a simple query through a mobile device application. These wearable computing technologies enable mobility and flexibility in situations of continuous health monitoring, generally speaking, in ambient assisted living (AAL) environments. These technologies have had a tremendous development in recent years and they play a very important and active role in the elderly surveillance. Monitoring systems are also able to assist people with different levels of intellectual and physical abilities and capacities. These solutions allow people to have a more "free" and active life style. They can offer great individual levels of independence, comfort, and active involvement in the community with security due to effective caring and continuous health monitoring and location. These approaches aim to provide significant improvements in terms of QoL, reducing interventions to support the elderly in their lifetimes. With the establishment of multi-agent systems it is possible to deploy new assistive technologies [5]. This paper presents a solution that provides a mobile health monitoring in AAL environments. This proposal makes use of a multi-agent system with self-configured interventions.

The considered mobile healthcare environment enables the identification of possible hazard situations or diseases. Furthermore, whenever possible, it makes a readjustment of long-term medical treatment based on the analysis of previous stored data. The mobile application can also perform an outdoor intervention in presence of possible accidents or risky situations. Thus reducing the intervention time of healthcare professionals or institutions. The stored health data and the monitored real time data allows an historical analysis of the collected parameters as well as an immediate response in emergency situations, such as falls or other risk situations.

The remainder of the paper is organized as follows. Section II elaborates on the related work, while Section III describes the system architecture overview. Section IV presents the used technologies while the mobile solution and its main features are addressed in Section V. Section VI performs a demonstration and evaluation of the proposed solution. Finally, the paper is concluded in Section VII. 


\section{Related Work}

Wearable and embedded body sensors collect biofeedback information for the human being. The great variety of sensors and the different ways that they can be applied on persons have contributed to several approaches regarding elderly people monitoring. These approaches mentioned in the literature use several techniques and technologies to capture biometric signals. This section presents several approaches that use body sensors in healthcare approaches for elderly people. Thereby, improving the elderly quality of life through several AAL solutions.

Kang et al. present a wrist-worn integrated health monitoring device who collects data of six bio-signals measuring, which includes fall detection, electrocardiogram (ECG), blood pressure, pulse oximetry ( $\mathrm{SpO} 2)$, respiration rate, and body surface temperature measuring [6]. This solution provides information concerning current person condition, such as vital bio-signals and location information. The developed system provides rapid and appropriate directions in emergency situations and alerts the user or caregiver to manage changes in health condition.

In [7], the authors present a proposal to capture bio-signals in healthcare and sport-training systems. The system uses infrared sensors or vision cameras. It includes a mobile threeaxis accelerometer motion system and smart shoes. The signals are measured and processed by a mobile device, allowing the analysis and diagnosis of postures during outdoor sports, as well as indoor activities. To improve the accuracy of the proposed motion capture, a frequency-adaptive sensor fusion method and a kinematic model are utilized to construct the whole body motion in real-time, and are continuously updated. Huang et al. present a wearing system with four sensors, ECG, three-axis accelerometer, temperature, and tight-switch, applied for remote monitoring system in home-care [8]. The ECG, measured with wearable electrodes using steel textile to generate the real-time heart-rate estimator. The heart-rate estimation is calculated to the movable textile electrodes in motion of user, performing a sophisticated work. The tightswitch sensor and FIR (Filter Impulse Response) filter technology are applied here to get the best heart-rate accuracy. The other biosensors can detect falls and body-temperature changes. Moreover, the device has low-power consumption to transmit detected bio-information from these four sensors and transmission turns it highly suitable for applications to remote healthcare and wellness.

In [9], the authors present a continuous monitoring of physiological parameters, such as, respiration, heart rate, temperature, and humidity. This solution aims to detect excessive sweating for health conditions, diagnostic reasons and the detection of life or health threatening events. This paper gives special attention to infants, especially those who cannot provide any oral or written feedbacks. A continuous monitoring under clinical and home conditions allows rescue calls as well as recognition of the development or progression of diseases at an early stage. Prototypes currently manufactured incorporate the chosen sensing principles with textile and textile-compatible technologies and are clinically tested for durability, handling, and signal quality.
Horta et al. presented a solution for fall detection in elderly people [10]. Falls can origin injuries that may cause a great dependence and even death in extreme cases. This system aims to prevent falls and advice the patient or even give instructions to treat an abnormal condition to reduce the risk of falling. Thus, this fall prevention system works in real time and the algorithms analyses bio-signals to thereby warn the user. Monitoring and processing data from sensors is performed by a smartphone that, in danger situations, can send eMails or SMSs (short message system) to a caretaker. The proposed solution was validated through a prototype and it is ready for use.

In [11], the authors propose the use of ECG, heart beat recognition, and peak detection that will offer the possibility to make diagnoses of heart diseases to prevent possible problems, through an "geometrical matching" rule evaluated and a local moving-window function procedure. This prevention is possible because of the Arrhythmia Database, which shows about $99 \%$ of positive identification for the R-waves through the low-order polynomial models. Rashidi and Mihailidis present a survey about the rapid emergence in assisted living technologies, against an aging society [12]. These technologies, tools, and techniques focus on biomedical data and an extensive range of healthcare applications to prevent possible situations of sickness. This information enables physicians to analyze the evolution of a patient and those trying to identify situations of stress or effort for a quick intervention in risk situations. They still look at current and future challenges, so give the elderly the comfort of their home and living independently.

Along with wearable and embedded sensors, a device that collects and saves bio-signals data, for example, during the absence of his caregiver is the Texas Instruments EZ430Chronos programmable clock [13]. Furthermore, another solution that allows data configuration collecting specific parameters and real time location while communicating with a mobile device is the use of Shimmer sensors technology [14].

The proposed solution gathers contributions from the above-described approaches. Next sections present it in detail.

\section{System Overview}

This section describes the system architecture considered for AAL environments with mobility support. Figure 1 illustrates a scenario with actions set for communications, in real time. Through an Internet connection, the mobile App allows data collection from sensors and external access from users terminal. It receives all the sensors data through Bluetooth to a smartphone or tablet. The collected data are analyzed and sent to a remote database. It can be stored for a more extensive analysis of the medication in order to improve or even adjust it. The body sensors and Web services modules facilitate the information sharing through a simple interface, which is properly identified by users with specified permissions. Therefore, the information sharing is safely and effectively performed. Measurements can be tuned according to different levels that caregiver defines most relevant. Moreover, this is a flexible solution allowing the integration of new sensors, if needed. 


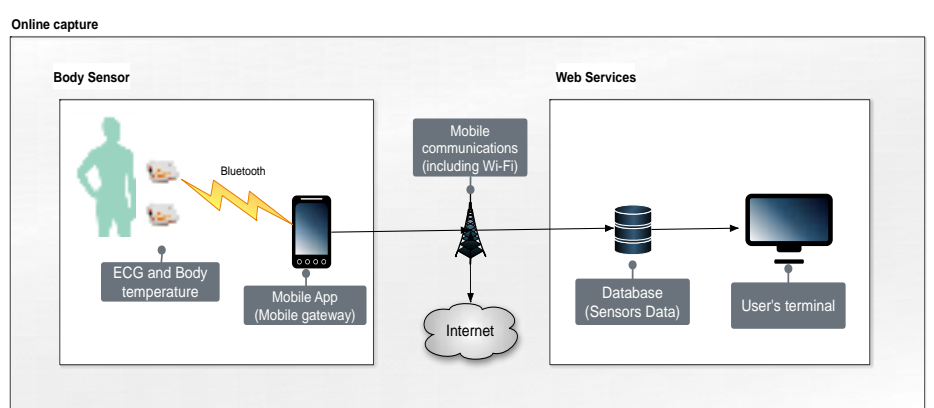

Fig. 1. Illustration of the system architecture, using ECG and temperature body sensors and external access through Web services for data visualization and storage.

Figure 2 presents the same approach but in offline mode. It stores data information to an offline database for later analysis. Offline bio-signal data is collected through radio frequency. Afterwards, a health care professional can analyze the stored data in order to verify whether the patient eventually needs any medication change or even another type of intervention.

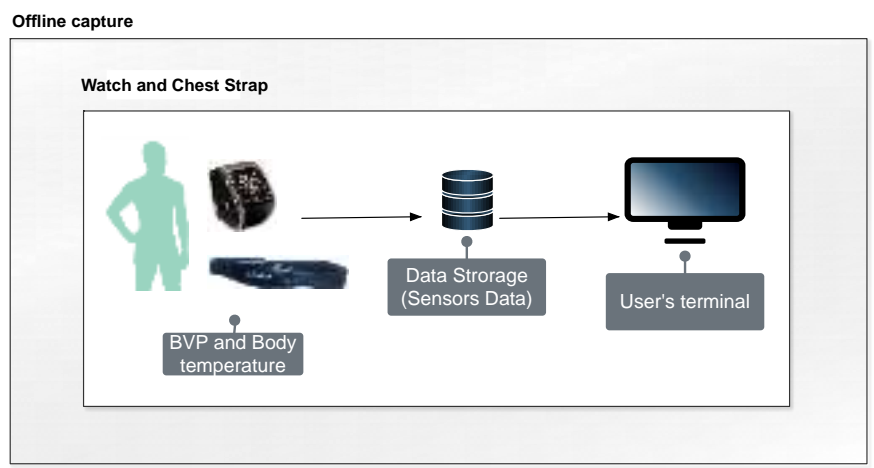

Fig. 2. Illustration of the offline system architecture considering the capture of blood volume pulse (BVP) and body temperature values.

Both presented architectures have common goals concerning the detection and analysis of bio-signals. They are responsible for detecting significant changes caused by common daily activities. These collected values can also infer significant changes of data through the sensors placed along the patients' body. The architecture presented in Figure 1 also allows inertial values collection that enables fall detections. This system includes the necessary amount of collected data to guarantee the well being of the elderly. It considers the interventions that a qualified healthcare team may find adequate for each user. So, there are two ways for collecting and storing data, either on-line or offline. Whether it is performed on-line (in real-time) or off-line, a later analysis points to the energetic efficiency of the various devices without compromising the reliability of biofeedback collected data.

\section{Used Technologies}

This section mentions the used technologies on the proposed system. Small instruments are able to collect and achieve data related to temperature, electrocardiogram (ECG), and blood volume pulse (BVP) also known as heart rate. Moreover, other sensors for bio-signals collection can be used, such as, electromyography (EMG), galvanic skin response (GSR), breathing, or the inertial signals as triple axis accelerometer, gyroscope, magnetometer, and altimeter. The sensors referred on this section include the MSP 430 microcontroller $(8 \mathrm{MHz}, 16 \mathrm{Bit})$ with Bluetooth socket, which allows communication and information processing in real time.

\section{A. Texas Instruments EZ430-Chronos}

The eZ430-Chronos watch, presented in Figure 3, needs disassembled to be reprogrammed with a custom application taking into account the needs of given users.

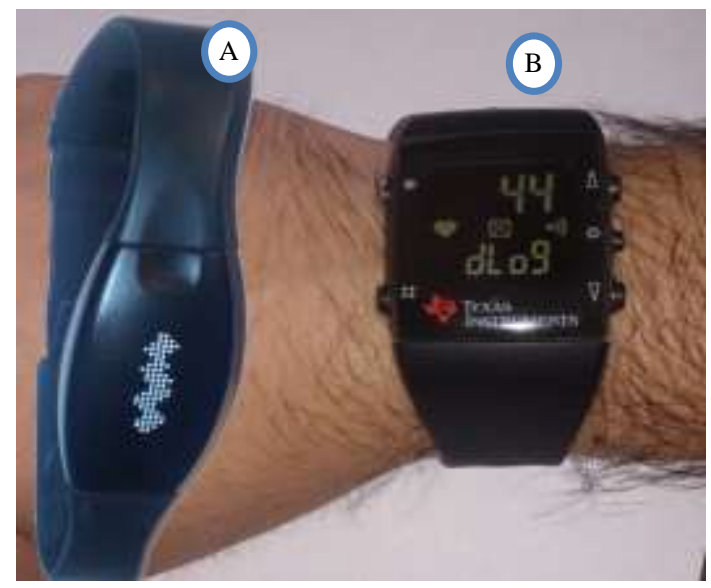

Fig. 3. Photo of the eZ430-Chronos watch: A) Chest Strap; B) Watch recording data (Temperature and Heart Rate).

The watch includes a 96-segment LCD display, an integrated pressure sensor, a temperature sensor, heart rate monitor through a chest strap, and a 3-axis accelerometer for motion sensitive control ability to operate on a central hub for nearby wireless sensors (such as pedometers). It was created an application that stores information about the temperature and heart rate from the patient's body using the chest strap. These instruments usually have a set of restrictions but, in this case, the watch performed well because it did not present any problems of flexibility, ergonomics, or discomfort resulting from time extended handling and use. Considering this, it is important to underline that the watch and chest strap autonomy are particularly large. During the whole process of acquiring data there was no need to change any batteries of either the watch or chest strap. For the CR2032 Coin Cell Lithium Battery, referred on the manual and according to its analysis, it was possible to observe that a continuous communication in BlueRobin Rx Mode (communication: chest strap to watch) the battery takes approximately 6.2 months, but if only need 1 hour of daily use, then, the battery works along 2 years. Otherwise, the SimpliciTI SYNC mode (communication: watch to computer) is more energetically costly because on continuous communication the battery takes about 8 days to be empty and with a 1 hour per day use it takes approximately 5.4 months. Despite the SimpliciTI SYNC mode has much higher energy costs, this is not a big problem because it is only used to send data to a terminal in this mode and this communication is performed in seconds.

\section{B. Shimmer Sensors}

Shimmer sensors offer two possibilities to communicate, the standard IEEE 802.15.4 or Bluetooth. Shimmers offer a plethora of body sensor bio-signals measurements, such as the 
ECG, electromyography (EMG), temperature, etc. It is also able to perform respiration demodulation on-chip. It integrates a 10 DoF inertial sensing via accelerometer, gyroscope, magnetometer, and altimeter, each one with selectable range. Each sensor is equipped with a rechargeable $450 \mathrm{mAh} \mathrm{Li-ion}$ battery, along with the advantage of being rechargeable within few hours.

In the proposal scenario seven types of sensors where used, as shown in Figure 4. The Shimmer ECG (A) records the electrical impulses through the heart muscle, having as starting point of four electrodes set $(\mathrm{C})$ on the chest of the patient. It is also possible to record data on bedridden or home users, or even during exercise to provide information of the patient's heart, in real time. Furthermore, a temperature sensor (B) allows the collection of a patient's temperature when the sensor is placed in close proximity to the body. The digital interface signal experimented several sensors for validation purposes.

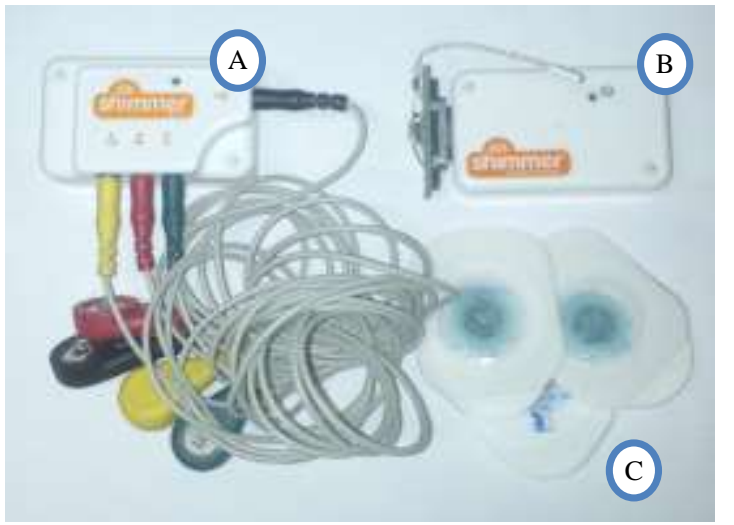

Fig. 4. Illustration of the body sensors - Shimmer: A) Electro-cardiogram; B) Temperature; C) Electrodes for Electro-cardiogram.

The Shimmer sensors can be applied to a wide range of scenarios, such as, heart function monitoring, premature ventricular contraction, atrial fibrillation, abnormal rhythm detection, fatigue analysis, muscle activity, sport technique, gait and posture disturbance, tremor analysis, and orthopedics biomechanics.

\section{Mobile Application}

The flexibility of the mobile devices enables more reliable and customizable mobile tools. Bio-signals monitoring allows the identification of possible and meaningful temperature, heart rate, or BVP changes. Additionally, the system can identify heart diseases like arrhythmia as well as other cardiac alterations. Moreover, the caregiver is able to define the risk degree of the patient under monitoring defining three levels of surveillance: low, normal, and critical [15-16].

This proposal allows a customizable and a non-invasive monitoring of ECG and temperature for a patient, making his/her daily life safer. Besides, it has the advantage of having a daily control in which all the information (numerical and graphical) is stored for future analysis whenever necessary. The mobile application behavior and procedures are presented in the activity diagram presented in Figure 5. This bio-signals monitoring solution use different Android activities to simultaneous tasks and several sensor readings. Therefore, the first step consists in the establishment of a Bluetooth connection. Once the connection is achieved, data collecting begins for ECG and temperature values. These are some considered sensors in this proposal. The collected data is analyzed and if it has expressive changes (for example, ECG values threshold per minute higher or irregular values), an alert is sent. In the same way the collected temperature is analyzed and if the values are very high or low, an alert is also sent to the elderly and/or to the caregiver.

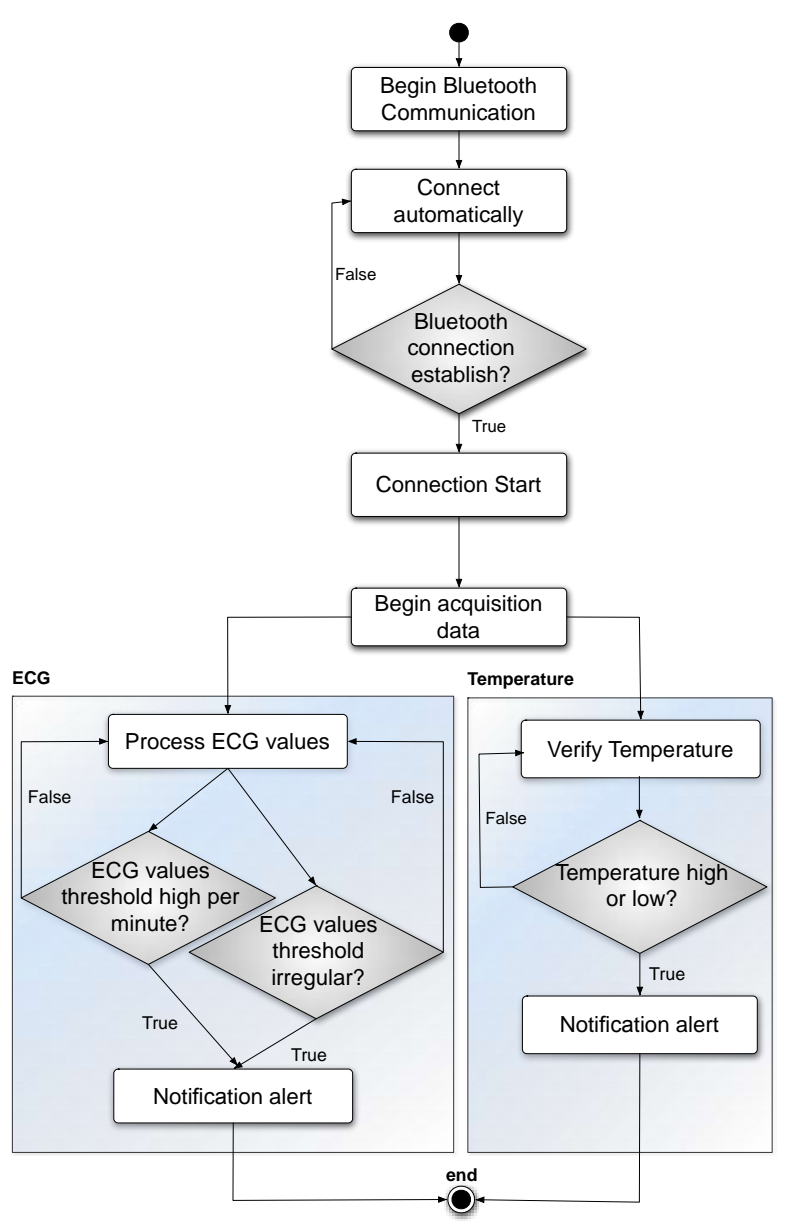

Fig. 5. Activity diagram illustrating the ECG and Temperature prevention, and detection procedures.

As above mentioned, there are several sensors that can be individually activated, such as, BVP, EMG, and GRS biosignals measurement, but also some inertial sensors as gyroscope and magnetometer. These sensors can also be present in a smartphone allowing the detection of significant changes in user motion. Therefore it enables fall detection through the accelerometer. The proposal has the ability to use the smartphone or tablet GPS to provide the real-time location of the user.

The presented proposal alerts immediately as possible both elderly and caregivers of significant changes that might raise life threatening situations. Moreover it stores data for a later analysis. These data can identify, for example, flu symptoms, heart diseases, such as a possible beginning of cardiac problems. 


\section{System Demonstration and Performance Evaluation}

This section presents the system demonstration and performance evaluation. Obtained results are presented considering two approaches for collecting biomedical data: the Texas Instruments programmable watch; and Shimmer sensors. Three levels of observation where considered: low for patients that are experiencing low levels of biomedical changes; normal for a regular patients; and critical level for real-time monitoring.

The demonstration and performance evaluation included five volunteers, aged from 20 to 30 years with no previous health records information. A set of experiments has been carried out for evaluate the system performance considering the three levels of surveillance. For each value of the abovementioned levels it was conducted a total of 30 trials distributed in three levels of alerts. On these trials it was collected and stored a total of 22 thousands samples of data.

\section{A. System Demonstration}

Figure 6 presents the gathered data (bio-signals) from one of the many experiments performed in this study. In each level, 10 samples were collected and analyzed afterwards. According to an average standard deviation of $10 \%$ and $15 \%$. The levels obtained in situations considered dangerous for the patient, were stored in a database. The graphic visualization is presented with various colors for different types of sensors.

Collecting biomedical data with the Texas Instruments programmable watch allows offline supervision at home or outdoors within a short distance. This approach was considered to be preventive or for future analysis of possible symptoms. The volunteers did not present any complaint regarding the lack of flexibility, ergonomics, or any discomfort resulting from time extended handling and use of the chest strap. After collecting data from the watch, it is possible to analyze the same (as may be seen in Figure 6), identifying the situations of stress or major concern. It is possible to calculate average values, such as, body temperature or heart rate values. Finally, it is feasible to define warning parameters for future analysis. Thus, a list of alert situations can be obtained and stored. At the end of the analysis, the caretaker can save the identified alerts according to the previous defined warning parameters.

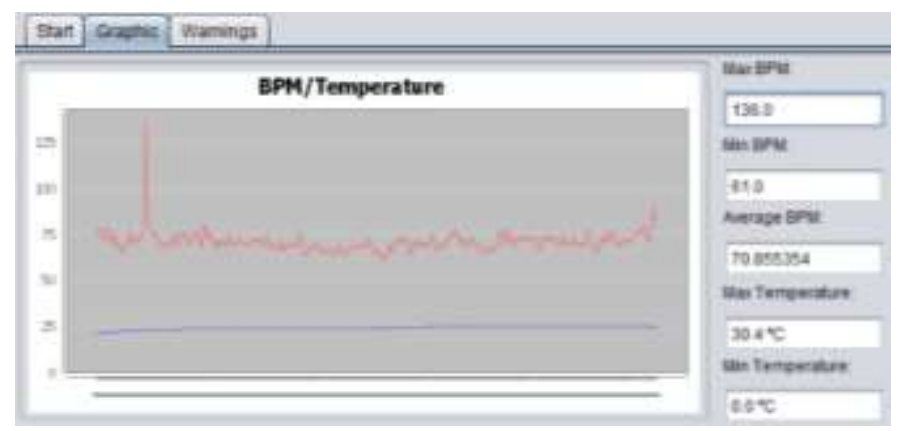

Fig. 6. Proposed application for the Texas Instruments watch showing collected data from temperature and heart rate from a given patient.

The normal monitoring level considers patients that have been recently ill or submitted to a surgery. In this context the monitoring process may occur in real time, however, with a gap time of 15 times per minute. This frequent monitoring identifies, changes on the body temperature, which may easily be a signal of an infection or an alteration of heart rate. However, these changes are not considered as "high risk" situations.

The critical monitoring level considers the surveillance of the patient's health, online and in real time. The data collection must be precise to immediately identify situations that might lead to the patient's death. In this case, patients must be in a hospital or another healthcare institution followed by qualified healthcare professionals. This allows the patient to have access to medical care and information as soon as possible.

This solution considers the collection of big amount of data. Therefore it is possible to recognize situations in that the patient health is in danger, online and in real time or by monitoring events offline. This way, it is possible to identify situations of disease, through body temperature alterations; high heart rates; premature ventricular contraction; abnormal rhythm detection; situations of cardiac arrhythmias and many other health situations that can be diagnosed.

\section{B. Performance Evaluation}

Several scenarios with the above-mentioned three levels of surveillance were considered to evaluate the performance of the proposed solution. Table I presents the total number of alerts per level of surveillance that shows the significant differences and improvements between levels of observation.

In the first observation level (low), volunteers were in a resting situation without making significant movements. Thus, emulating a situation of a bedridden elderly or an elderly with great physical limitations. In the second level (normal) the volunteers could have some movements performing daily tasks. This way, representing an elderly who is able to perform some of the household chores. The third level (critical) is for cases in which patients show signs of possible risky situations. In this level, all the variations of biomedical signals are considered dangerous for the well-being and for the health of the elderly.

TABLE I. TOTAL NUMBER OF ALERTS PER LEVEL OF SURVEILlANCE (LOW, NORMAL AND CRITICAL) WITH A STANDARD DEVIATION OF 10\% AND 15\% CONSIDERING THE TI WATCH AND SHIMMER SENSORS, FOR THE 30 EXPERIMENTS.

\begin{tabular}{|c|c|c|c|c|}
\cline { 2 - 5 } \multicolumn{1}{c|}{} & \multicolumn{2}{c|}{ Texas Instruments watch } & \multicolumn{2}{c|}{ Shimmer sensors } \\
\cline { 2 - 5 } \multicolumn{1}{c|}{} & Level 1 (10\%) & Level 2 (15\%) & Level 1 (10\%) & Level 2 (15\%) \\
\hline Low & 64 & 39 & 32 & 1 \\
\hline Normal & 473 & 170 & 134 & 53 \\
\hline Critical & 1754 & 788 & 504 & 250 \\
\hline
\end{tabular}

The Shimmer sensors include little alerts, presenting more accurate data and resulting in an error reduction when increasing the standard deviation. Moreover, when alerts occurred, their time analysis was also important. When there is an alert in only one of the samples (in time), it is not considered meaningful. The alerts in one sample can be a misreading and it is only considered as meaningful and dangerous situation after several consecutive alerts. 


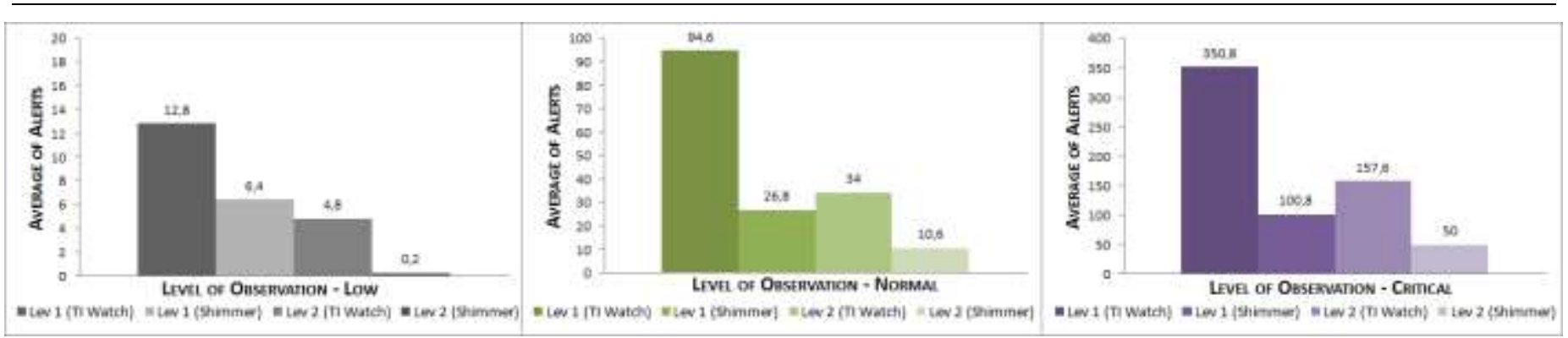

Fig. 7. Comparison of average alerts values to each level of observation system (Watch and Shimmer).

Figure 7 presents the comparison of average alert values to each level of observation. With the decrease and exclusion of possible errors, the results improve about $2 \%$, for the Texas Instruments watch and about $0.8 \%$ for the Shimmer sensors. Furthermore, it is observed that various levels of observation have different amounts of alerts increasing the surveillance level. The Shimmer sensors have more capacity to read data in equal time intervals and in a worst-case scenario they are capable to detect a greater number of alert situations.

\section{Conclusion and Future Work}

The presented mobile healthcare solution for AAL environments improves the lives of elderly people that live alone or in remote locations. It allows continuous assistance and monitoring of bio-signals to identify potential diseases or health risk situations. The eZ430-Chronos watch was used for prevention in an online and offline mode. According to the online analysis, Shimmer sensors are with no doubt an improvement allowing the collection of data within seconds. The use of mobile devices, such as, smartphones or tablets were considered for sending alerts in case of critical situations through data transmitted from various sensors and for mobility purposes. The solution is customizable and only the sensors required are enabled. Also, fall detection and real-time location are deployed. This proposed solution is extremely easy to place on the patient's body, flexible, and with no harm to his health. System demonstration and performance evaluation shows more precise results and increase the exclusion of possible error results about $2 \%$, and about $0.8 \%$, were obtained by the Texas Instrument watch, and the Shimmer sensors respectively.

Future work considers the deployment of two more types of sensors to analyze the patient's posture that will had a great value and a wider meticulousness on healthcare assistance to the elderly, all of these without compromising their flexibility of movements and autonomy or security.

\section{Acknowledgments}

Part of this work has been supported by Instituto de Telecomunicações, Next Generation Networks and Applications Group (NetGNA), Portugal, by Government of Russian Federation, Grant 074-U01, by National Funding from the FCT - Fundação para a Ciência e a Tecnologia through the PEst-OE/EEI/LA0008/2013 Project, and by the AAL4ALL (Ambient Assisted Living for All), project co-funded by COMPETE under FEDER via QREN Programme.

\section{References}

[1] World Health Organization, "Disability and health", http://www.who.int/mediacentre/factsheets/fs352/en/, June 2011.
[2] Eurostat, Demography Report 2010 "Older, more numerous and diverse Europeans", Publications Office of the European Union, 2011.

[3] U.S. Department of Health and Human Services, "Profile of Older Americans: 2011", http://www.aoa.gov/Aging_Statistics/Profile/2011/docs/2011profile.pdf.

[4] T. Shimokakimoto and K. Suzuki, "A chair-type interface for long-term and ambient vital sensing", Annual International Conference of the IEEE, Engineering in Medicine and Biology Society (EMBC 2011), pp. 1173-1176, Aug. 30 - Sept. 3, 2011.

[5] J. J. McNaull, J. C. Augusto, M. Mulvenna and P. McCullagh, "Multiagent Interactions for Ambient Assisted Living", 7th International Conference on Intelligent Environments (IE 2011), Nottingham, UK, pp. 310-313, 25-28 July 2011.

[6] J. M. Kang, T. Yoo, H. and C. Kim, "A Wrist-Worn Integrated Health Monitoring Instrument with a Tele-Reporting Device for Telemedicine and Telecare", Instrumentation and Measurement, IEEE Transactions, vol. 55, pp. 1655-1661, Oct. 2006.

[7] P.Jung, G. Lim and K, Kong, "A mobile motion capture system based on inertial sensors and smart shoes", 2013 IEEE International Conference on Robotics and Automation (ICRA 2013), Karlsruhe, Germany, pp. 692-697, 6-10 May 2013.

[8] W. Huang, C. Chen, Y. Chang, Y. Chen, J. Huang, C. M.Yang and T.L. Yang, "Exquisite textiles sensors and wireless sensor network device for home health care", 30th Annual International Conference of the IEEE Engineering in Medicine and Biology Society (EMBS 2008), pp. 546549, 20-25 Aug. 2008.

[9] C. Linti, H. Horter, P. Osterreicher and H. Planck. "Sensory Baby Vest for the Monitoring of Infants", Proceedings of the International Workshop on Wearable and Implantable Body Sensor Networks, Cambridge, MA, pp. 135-137, 3-5, April 2006.

[10] E. T. Horta, I. C. Lopes, J. J. P. C. Rodrigues and S. Misra, "Real time falls prevention and detection with biofeedback monitoring solution for mobile environments", 2013 IEEE 15th International Conference eHealth Networking on Applications \& Services (IEEE Healthcom 2013), Lisbon, Portugal, pp. 594-600, 9-12. Oct. 2013.

[11] K. V. Suárez, J. C. Silva, Y.Berthoumieu, P. Gomis, and M. Najim, "ECG beat detection using a geometrical matching approach", IEEE Transactions on Biomedical Engineering, vol. 54, pp. 641-650, April 2007.

[12] P. Rashidi and A. Mihailidis, "A Survey on Ambient-Assisted Living Tools for Older Adults", IEEE Journal of Biomedical and Health Informatics, vol. 17, pp. 579-590, May 2013.

[13] Texas Instruments, "EZ430-Chronos", 2014 [Online]. Available: http://processors.wiki.ti.com/index.php/EZ430-Chronos; Accessed: May 2014.

[14] Shimmer, "Shimmers Sensors", 2014 [Online]. Available: http://www.shimmersensing.com/; Accessed: May 2014.

[15] M. V. Ramesh, S. Anand and P. Rekha, "A mobile software for health professionals to monitor remote patients", Ninth International Conference on Wireless and Optical Communications Networks (WOCN 2012), pp. 1-4, 20-22 Sept. 2012.

[16] J. J. Oresko, J. Zhanpeng, J. Cheng, S. Huang, Y. Sun, H. Duschl and A.C. Cheng, "A Wearable Smartphone-Based Platform for Real-Time Cardiovascular Disease Detection Via Electrocardiogram Processing", IEEE Transactions on Information Technology in Biomedicine, vol. 14, pp. 734-740, May 2010. 
